MANUALI

SCIENZE SOCIALI

$-8-$ 

LEONARdo MaRChETTONI

\title{
Breve storia della democrazia
}

\author{
Da Atene al populismo
}

Firenze University Press

2018 
Breve storia della democrazia : da Atene al populismo / Leonardo Marchettoni. - Firenze : Firenze University Press, 2018.

(Manuali. Scienze Sociali ; 8)

http://digital.casalini.it/9788864537610

ISBN 978-88-6453-760-3 (print)

ISBN 978-88-6453-761-o (online PDF)

ISBN 978-88-6453-762-7 (online EPUB)

Immagine di copertina: (C) Alberto Pizarro Fernández

Il volume è pubblicato con fondi iscritti nel bilancio del Dipartimento di Giurisprudenza, di Studî Politici e Internazionali dell'Università degli Studi di Parma.

Certificazione scientifica delle Opere

Tutti i volumi pubblicati sono soggetti ad un processo di referaggio esterno di cui sono responsabili il Consiglio editoriale della FUP e i Consigli scientifici delle singole collane. Le opere pubblicate nel catalogo della FUP sono valutate e approvate dal Consiglio editoriale della casa editrice. Per una descrizione più analitica del processo di referaggio si rimanda ai documenti ufficiali pubblicati sul catalogo on-line della casa editrice (www.fupress.com).

Consiglio editoriale Firenze University Press

A. Dolfi (Presidente), M. Boddi, A. Bucelli, R. Casalbuoni, M. Garzaniti, M.C. Grisolia, P. Guarnieri, R. Lanfredini, A. Lenzi, P. Lo Nostro, G. Mari, A. Mariani, P.M. Mariano, S. Marinai, R. Minuti, P. Nanni, G. Nigro, A. Perulli, M.C. Torricelli.

La presente opera è rilasciata nei termini della licenza Creative Commons Attribution 4.0 International (CC BY 4.0: http://creativecommons.org/licenses/by/4.o/legalcode).

This book is printed on acid-free paper

CC 2018 Firenze University Press

Università degli Studi di Firenze

Firenze University Press

via Cittadella, 7, 50144 Firenze, Italy

www.fupress.com

Printed in Italy 
Questo libro è dedicato alla memoria di Danilo Zolo, maestro di sobrietà, di rigore e di passione 



\section{Sommario}

Cap. 1 - La democrazia ateniese

1. Democrazia: continuità e cambiamento 3

2. Origini della democrazia ateniese 6

3. Le istituzioni della democrazia ateniese 7

4. Le ragioni dell'estrazione a sorte 10

5. Esercito e dinamiche sociali 11

6. Prime testimonianze sulla democrazia 12

7. Platone 13

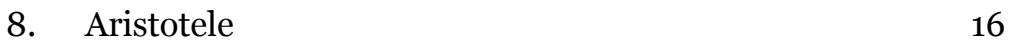

Cap. 2 - Democrazia e modernità $\quad 21$

1. Il modello romano 21

2. Modelli di giustificazione del potere nel Medioevo 22

3. La partecipazione popolare nei comuni 25

4. Teorizzare l'autonomia $\quad 27$

5. Il recupero della tradizione repubblicana 28

6. Origini dello Stato 30

7. I livellatori e la prima rivoluzione inglese 31

8. Il modello contrattualista 33

9. La Glorious Revolution e Locke 36

10. Spinoza 39

11. Montesquieu 41

12. Rousseau 42 
Cap. 3 - L'Ottocento 47

1. La rivoluzione americana 47

2. La rivoluzione francese 50

3. Dopo la rivoluzione 52

4. Constant e Tocqueville 53

5. Bentham e John Stuart Mill $\quad 56$

6. Kant 57

7. Fichte e Hegel 59

8. Marx 62

Cap. 4 - Il Novecento 65

1. Tra Ottocento e Novecento 65

2. Weber 66

3. La crisi della sovranità popolare e l'elitismo $\quad 69$

4. Dopo la prima guerra mondiale $\quad 72$

5. Il secondo dopoguerra $\quad 74$

6. Rawls 76

7. Hayek, Nozick e la tradizione neoliberale $\quad 78$

8. La teoria critica e Habermas 80

9. Foucault 83

Cap. 5 - La democrazia costituzionale $\quad 87$

1. I limiti della democrazia $\quad 87$

2. I diritti fondamentali $\quad 89$

3. Costituzioni e pluralismo $\quad 91$

4. Parlamenti e partiti 95

5. Partiti e comunicazione nel secondo dopoguerra 97

6. Partiti e pluralismo 100

Cap. 6 - Crisi e trasformazioni della democrazia 103

1. Elementi di crisi della democrazia 103

2. L'alternativa deliberativa 106

3. Populismo 109

Conclusioni. Democrazia e verità 115

$\begin{array}{ll}\text { Bibliografia } & 121\end{array}$ 


\section{Presentazione}

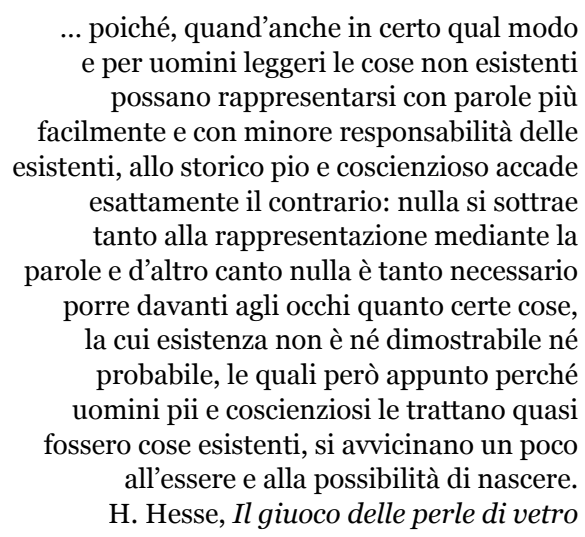

Apprestandomi a concludere questo lavoro mi rendo conto che un'esecuzione ideale del progetto avrebbe richiesto una conoscenza approfondita della storia del pensiero politico, oltre a una completa padronanza dei diversi contesti storici, dell'evoluzione delle istituzioni, e via dicendo. $\mathrm{Si}$ tratta evidentemente di un traguardo che probabilmente non si può sperare di raggiungere neppure al termine di un'operosa carriera di studioso. Non c'è bisogno di dire che non posseggo simili competenze.

Come è inevitabile che sia, la selezione degli argomenti e degli autori è determinata da un coacervo di fattori contingenti - conoscenze pregresse, preferenze, intenti - all'interno di un auspicio generale di leggere la storia della democrazia sullo sfondo della storia del pensiero politico e della storia delle istituzioni. Questa circostanza mi porta retrospettivamente a vedere il mio lavoro, non come il tentativo di fornire una descrizione, più o meno accurata, di un continente già noto, ma come un esperimento volto a formulare congetture sulle caratteristiche salienti di un territorio in larga parte inesplorato, estrapolandole dagli sparsi dati in mio possesso. Se si porta all'estremo questo punto di vista, cambia la natura stessa del compito che ci si propone: non un'impresa ricostruttiva, ma un tentativo costruttivo; più che scoprire e descrivere qualcosa che esiste già, immaginare un sistema di nessi e relazioni che possa risultare persuasivo.

La scelta e l'organizzazione del materiale tradisce l'origine e la destinazione di questa Breve storia della democrazia. Il testo nasce come supporto del Corso di Analisi delle Istituzioni Politiche che tengo dall'a.a. 2014-2015 presso l'Università di Parma. In questi anni mi sono valso di svariati eccellenti strumenti didattici, tra cui mi piace ricordare Demo- 
crazia di Stefano Petrucciani, che per molti versi rappresenta il modello che ho maggiormente tenuto presente nella selezione degli argomenti.

Diversi colleghi e amici mi hanno inviato osservazioni e commenti, segnalando errori e omissioni: Luca Baccelli, Thomas Casadei, Emanuele Castelli, Pietro Costa, Dimitri D’Andrea, Francesco De Vanna, Gianluigi Palombella, Lucia Re, Emilio Santoro, Italo Testa. A tutti loro il mio più sentito ringraziamento. Ringrazio inoltre i due anonimi referees della Firenze University Press.

Infine, mi sia consentito rivolgere un affettuoso pensiero a mio padre Lorenzo, ai miei figli Lorenzo, Francesco e Alessandro e a mia moglie Patrizia, che mi hanno sostenuto durante la stesura di queste pagine.

Balatonfenyves, agosto 2018 


\section{Capitolo primo \\ La democrazia ateniese}

\section{Democrazia: continuità e cambiamento}

Il termine 'democrazia' ha origini greche - da demos e kratos = 'governo del demos' - e indica una delle tre basilari forme di governo, vale a dire una delle modalità in cui il potere politico viene esercitato e, quindi, uno dei modelli fondamentali di organizzazione del sistema politico': monarchia - in cui il governo è in mano a uno solo -, aristocrazia - in cui il governo è in mano a pochi, eminenti per ascendenza - e democrazia appunto - in cui il governo è in mano a molti.

Peraltro, vale la pena notare come il riferimento al demos contenga una potenziale ambiguità: da una parte, il demos rappresenta l'intero corpo dei cittadini, quindi è termine tendenzialmente includente e avalutativo, che definisce un insieme di persone privo di un'identità specifica; dall'altra, connota più precisamente il popolo, in contrasto con le fazioni nobiliari. Quindi è termine che comporta potenziali esclusioni e si presta a un uso valutativo ${ }^{2}$. I membri del demos, in questa seconda accezione, sono portatori di un'identità. Inoltre, il riferimento al kratos, che significa 'potere', 'potenza' ma anche 'violenza' è probabilmente spia di un'originaria accezione negativa, che coglieva gli aspetti di prevaricazione sulla legge insiti nel governo del popolo ${ }^{3}$.

${ }^{1}$ Cfr.: N. Bobbio, Stato, governo, società. Frammenti di un dizionario politico, Einaudi, Torino 1995, pp. 95 sgg.; A. Barbera, C. Fusaro, Il governo delle democrazie, il Mulino, Bologna 2009.

${ }^{2}$ Vedi: M.H. Hansen, The Athenian Democracy in the Age of Demosthenes, Duckworth, London 1999, p. 334; R. Osborne, Athens and Athenian Democracy, Cambridge University Press, Cambridge 2014, pp. 39 sgg.

3 Vedi: C. Mossé, Pericle. L’inventore della democrazia (ed. or. 2005), Laterza, Roma-Bari 2006, p. 60; L. Canfora, Se la democrazia degli antichi sia ancora 
Oggetto di questa primo capitolo è la democrazia ateniese4 ${ }^{4}$ Tuttavia, prima di entrare nello specifico, può essere utile premettere alcune brevissime distinzioni introduttive ${ }^{5}$. La prima distinzione importante è quella tra democrazia diretta e democrazia rappresentativa. La democrazia diretta è una forma di governo nella quale i cittadini amministrano direttamente la comunità politica, riunendosi in assemblea e prendendo le decisioni più importanti - non necessariamente approvando le leggi - come nell'Atene del V-IV secolo a.C. Nella democrazia rappresentativa - come in tutte le democrazie contemporanee - $\mathrm{i}$ cittadini eleggono dei rappresentanti che eserciteranno il potere di fare le leggi.

Democrazia non è sinonimo di sovranità popolare. Infatti, detto «sovranità» il sommo potere connesso all'esercizio delle funzioni di ogni sistema politico, la teoria della sovranità popolare asserisce che il sommo potere politico appartiene al popolo. Come tale essa coglie una delle caratteristiche specifiche degli Stati moderni in cui il sommo potere, che coincide con il potere legislativo, deriva la propria legittimazione dal fatto di essere esercitato da rappresentanti del popolo ${ }^{6}$.

Coloro che sono attivi in democrazia sono i cittadini, vale a dire i soggetti detentori della cittadinanza. L'insieme dei cittadini non coincide ovviamente con l'insieme degli individui residenti in un determinato territorio, in quanto i cittadini sono definiti dal possesso di determinati requisiti attinenti all'età, al sesso, all'origine, al censo ecc. Allo status di cittadini corrispondono determinate facoltà e incombenze, determinati diritti e doveri, per usare due termini moderni che, in rapporto alla democrazia ateniese, risulterebbero anacronistici ${ }^{7}$.

Di democrazia oggi si parla in molti sensi: si può distinguere tra democrazia formale, attinente all'uguaglianza giuridica e alle procedure messe in atto, e democrazia sostanziale, attinente alle condizioni di uguaglianza sociale ed economica, che dovrebbero costituire la premessa e il risultato

utile per emendare quella dei moderni, in C. Altini (a cura di), Democrazia. Storia e teoria di un'esperienza filosofica e politica, il Mulino, Bologna 2011, pp. 63-80: 63-64.

${ }^{4}$ Sul tema della democrazia ateniese due testi compatti e maneggevoli sono: D. Stockton, The Classic Athenian Democracy, Oxford University Press, Oxford 1990 e J. Thorley, Athenian Democracy, Routledge, London-New York 2004. Lo studio più approfondito è però Hansen, The Athenian Democracy in the Age of Demosthenes, cit. Vedi inoltre i saggi contenuti in D. Musti, Demokratía. Origini di un'idea, Laterza, Roma-Bari 1995 e i capitoli dedicati alla democrazia greca in P. Cartledge, Democracy: A Life, Oxford University Press, Oxford 2016.

5 Per una trattazione introduttiva al lessico democratico, si può vedere il capitolo Democrazia e dittatura, in Bobbio, Stato, governo, società, cit.

${ }^{6}$ Cfr. N. Matteucci, Lo Stato moderno, il Mulino, Bologna 1993, cap. 2.

7 Sul tema, cfr.: P. Costa, Cittadinanza, Laterza, Roma-Bari 2005; É. Balibar, Cittadinanza, Boringhieri, Torino 2012. 
dei processi democratici ${ }^{8}$. Ancora, di democrazia oggi si parla anche al di fuori dell'ambito più strettamente politico, per fare riferimento a un metodo per prendere decisioni nel mondo del lavoro, della scuola ecc. - il datore di lavoro che decide di concerto con i dipendenti, l'insegnante con gli studenti, e via dicendo. In questo senso si ha riguardo ai processi di democratizzazione della società 9 .

Tornando alla democrazia classica, il primo problema da porsi consiste nel domandarsi se sussista o meno un filo di continuità con la democrazia contemporanea. Ha senso porsi il problema di un nucleo immutabile della democrazia? Ha senso chiamare la democrazia greca «democrazia»? Per un verso, infatti, le istituzioni della democrazia greca erano completamente diverse dalle nostre. Inoltre, la democrazia greca prevedeva una partecipazione estremamente limitata, visto che non erano cittadini né le donne, né gli stranieri, né gli schiavi. D'altra parte, si può anche osservare che tutto cambia: il sole, l'acqua che guardavano i greci non sono il sole e l'acqua che guardiamo noi. Per esempio, oggi pensiamo che il sole sia una stella, distante circa 150 milioni di chilometri, composta in massima parte da elio e idrogeno, in cui avvengono reazioni termonucleari ecc. Come pensiamo che ci sia identità tra il sole dei greci e il nostro, così, autorizzati da una continuità lessicale, possiamo usare il termine 'democrazia' per parlare del loro sistema politico, come del nostro ${ }^{10}$.

Vi è poi un'altra considerazione, di natura 'pragmatica' che ci viene in soccorso e che può essere mobilitata in risposta alla domanda sul perché la conoscenza della democrazia ateniese sia rilevante per la comprensione della democrazia 'dei moderni', anche se i quadri socioeconomico e istituzionale sono mutati così profondamente. Si tratta del fatto che i problemi discussi dagli antichi in rapporto al governo democratico si ritrovano nei secoli successivi, segnando l'agenda del dibattito filosoficopolitico occidentale. Per tutti questi motivi, vale la pena di interrogarsi ancora sulla forma di governo dell'Atene classica e di riferirsi a essa con il nome di 'democrazia'"

${ }^{8}$ Vedi Bobbio, Stato, governo, società, cit., pp. 149-150.

9 Ivi, pp. 146-148.

${ }^{10}$ Giustificare in modo minimamente articolato queste proposizioni richiederebbe un lunghissimo excursus di contenuto epistemologico-semantico che qui non è il caso di intraprendere. Sul tema, mi permetto di rinviare a L. Marchettoni, Relativismo e differenza culturale, Nuova Cultura, Roma 2012.

${ }_{11}$ Su questo tema, cfr.: M.I. Finley, La democrazia degli antichi e dei moderni (ed. or. 1985), Laterza, Roma-Bari 2010; Canfora, Se la democrazia degli antichi sia ancora utile per emendare quella dei moderni, cit. Uno dei più noti sostenitori della tesi 'continuista' è Robert Dahl, cfr. R. Dahl, La democrazia e i suoi critici (ed. or. 1989), Editori Riuniti, Roma 1990. Va detto che altri autori hanno scelto soluzioni diverse. Per esempio, secondo Bernard Manin, democrazie in senso proprio sono soltanto le democrazie dirette. Le forme di governo moderne, basate 


\section{Origini della democrazia ateniese}

L'origine della democrazia si pone al culmine di un processo secolare di trasformazione della società greca, dopo l'eclissi del potere dei re-sacerdoti e l'ascesa delle poleis. Il contesto economico e sociale è segnato da instabilità dovute a ripetute ondate migratore, progressi tecnici nella navigazione, intensificazione degli scambi commerciali, fondazione di colonie. La polis racchiude una comunità, organizzata intorno all'acropoli - l'area dei templi - e all'agorà - la piazza - che progressivamente acquisisce consapevolezza del proprio carattere specifico. In un primo tempo il governo della polis è in mano alle famiglie aristocratiche, che devono la loro ricchezza al possesso della terra, ma in seguito le altre fasce della popolazione reclamano un miglioramento della loro condizione e un maggior coinvolgimento nella gestione della cosa pubblica ${ }^{12}$.

Questa vicenda è evidente nella storia di Atene. Atene non fu sicuramente l'unica polis organizzata democraticamente ma, in virtù del suo prestigio, è quella di cui conosciamo meglio l'organizzazione. Ad Atene Solone, eletto arconte, vale a dire sommo magistrato, all'inizio del VI secolo a.C., pose mano a una riforma complessiva dell'organizzazione politica e sociale della città, distinguendo quattro fasce di censo, pentacosiomedimni, cavalieri, zeugiti e teti, a cui corrispondevano diversi gradi di partecipazione alla vita politica - solo le prime tre classi potevano accedere alle magistrature, ma anche i teti potevano partecipare all'assemblea. Inoltre, Solone varò diverse misure volte a indebolire le strutture del potere aristocratico: in particolare, abolì la schiavitù per debiti e ridusse il potere dell'Areopago, l'organo attraverso il quale gli esponenti delle famiglie nobiliari avevano sino ad allora governato Atene ${ }^{13}$.

Gli anni successivi alla riforma di Solone sono segnati prima dalla parentesi della tirannide di Pisistrato, il quale governò Atene, con alcune interruzioni, dal 560 a.C. circa al 527 a.C., senza peraltro cancellare, almeno formalmente, le riforme di Solone, e in seguito da una fase storica convulsa, coincidente con il tentativo dei figli di Pisistrato, Ippia e

sull'espediente della rappresentanza vanno piuttosto qualificate come «governi rappresentativi». Cfr. B. Manin, Principî del governo rappresentativo (ed. or. 1997), il Mulino, Bologna 2010.

${ }^{12}$ Sul concetto di polis, cfr. G. Cambiano, Polis. Un modello per la cultura europea, Laterza, Roma-Bari 2000. Sulle origini della democrazia ateniese, cfr. Cartledge, Democracy, cit., pp. 49-59.

${ }_{13}$ Vedi: Stockton, The Classic Athenian Democracy, cit., pp. 19-21; Hansen, The Athenian Democracy in the Age of Demosthenes, cit., pp. 29-32, 43-46; Thorley, Athenian Democracy, cit., pp. 11-17. 
Ipparco, di perpetuare il potere del padre ${ }^{14}$. Infine, l'assetto democratico fu ristabilito negli ultimi anni del secolo - nel 508-507 a.C. - da Clistene.

\section{Le istituzioni della democrazia ateniese}

Clistene, senza detenere la carica di arconte, mise mano a una radicale riforma sociale e politica. Questo fu possibile, nonostante l'opposizione della maggior parte dell'aristocrazia, grazie all'appoggio popolare e a quello dei membri della famiglia degli Alcmeonidi - esiliati durante la tirannia di Pisistrato ${ }^{15}$.

Clistene abolì le quattro tribù censitarie istituite da Solone e ripartì i cittadini in 10 tribù territoriali. Tutta la regione su cui Atene esercitava il proprio controllo, l'Attica, fu suddivisa in tre aree: la città, la costa e le zone interne. A sua volta, ciascun'area era suddivisa in demi - 139 in totale. Ciascuna tribù, infine, constava di un numero variabile di demi, scelti tra le tre aree. Il risultato era che ciascuna tribù risultava dall'aggregazione di demi della città, demi costieri e demi delle zone interne. Questa macchinosa architettura era finalizzata a produrre un rimescolamento della cittadinanza, rompendo l'unità delle vecchie tribù fondate sul censo ${ }^{16}$.

Soprattutto, Clistene rinnovò radicalmente le istituzioni politiche della città. In seguito alla sua riforma la democrazia ateniese raggiunge la sua fisionomia classica. Le istituzioni principali erano le seguenti:

- Ekklesía, assemblea di tutti i cittadini ${ }^{17}$. Si riuniva prima nell'agorà e poi nella Pnice, una collina nei pressi dell'Acropoli. Si tenevano circa 40 sedute ogni anno ${ }^{18}$. Erano cittadini soltanto i maschi, maggiori di venti anni, con padre ateniese (nel 451-450

${ }^{14}$ Le testimonianze ci informano che Pisistrato governò in modo equilibrato, assicurando stabilità e prosperità ad Atene. Continuò, in effetti, l'opera di Solone, limitando la conflittualità e le ambizioni di potere delle famiglie aristocratiche esiliando i membri della potente famiglia degli Alcmeonidi - e introducendo misure di sostegno a favore dei piccoli agricoltori. Vedi: Stockton, The Classic Athenian Democracy, cit., pp. 21-22; Hansen, The Athenian Democracy in the Age of Demosthenes, cit., pp. 32-33; Thorley, Athenian Democracy, cit., pp. 17-22.

${ }_{15}$ Per una panoramica sull'evoluzione della democrazia ateniese negli anni di Clistene, cfr. Cartledge, Democracy, cit., pp. 61-75.

${ }^{16}$ Vedi: Stockton, The Classic Athenian Democracy, cit., pp. 57-67; Hansen, The Athenian Democracy in the Age of Demosthenes, cit., pp. 34-36, 46-49; Thorley, Athenian Democracy, cit., pp. 24-28.

${ }_{17}$ Sull'ekklesía in generale vedi: Stockton, The Classic Athenian Democracy, cit., pp. 67-84; Hansen, The Athenian Democracy in the Age of Demosthenes, cit., pp. 125-160; Thorley, Athenian Democracy, cit., pp. 32-34.

${ }^{18}$ Sul tema cfr. E.M. Harris, Democracy and the Rule of Law in Classical Athens: Essays on Law, Society, and Politics, Cambridge University Press, Cambridge 2006, pp. 80-120. 
a.C. Pericle fece approvare una legge più restrittiva che richiedeva che entrambi i genitori fossero ateniesi). Erano esclusi quindi i minori, le donne, gli stranieri, gli schiavi. Nel quinto-quarto secolo i cittadini erano 30.000-60.000 su una popolazione complessiva di 250.000-300.000 abitanti ${ }^{19}$. Però, coloro che partecipavano all'assemblea erano solo poche migliaia: la Pnice poteva accogliere circa 6.000 persone e, inoltre, secondo alcuni autori, la partecipazione usuale si attestava sulle 2.000-3.000 unità ${ }^{20}$. La scarsa partecipazione aveva sicuramente ragioni logistiche: infatti i cittadini che abitavano le regioni dell'Attica più lontane da Atene avrebbero avuto difficoltà a recarsi alle sedute dell'assemblea. Più controverso è stabilire quanto gli abitanti di Atene fossero interessati a partecipare ${ }^{21}$. In ogni caso, le decisioni più importanti venivano approvate con il voto di circa un quarantesimo/un cinquantesimo degli abitanti complessivi della regione. I compiti dell'ekklesía erano molto vasti: 'politica estera', amministrazione della città, elezione delle principali magistrature, ostracismo $^{22}$, giudizi di particolare rilevanza ${ }^{23}$. Tutti avevano diritto di prendere la parola (isegoría) ma in pratica solo coloro che avevano ricevuto una formazione da oratore coglievano questa opportunità ${ }^{24}$. Una seria limitazione all'isegoría fu introdotta nel 462 a.C. con l'istituto del grafē paranómōn. Il cittadino che in assemblea avesse proposto alcunché in contrasto con il diritto esistente o con gli interessi del popolo poteva essere condannato

19 Secondo Hansen il numero dei cittadini raggiunse un picco attorno al 430 a.C. prima di venir dimezzato dalla peste. Nel quarto secolo il numero dei cittadini rimase più o meno stabile, intorno alle 30.000 unità. Vedi Hansen, The Athenian Democracy in the Age of Demosthenes, cit., pp. 327-328. Il numero complessivo degli abitanti è difficile da stimare perché non abbiamo dati certi riguardo al numero degli schiavi. Vedi Musti, Demokratía, cit., p. 147.

${ }^{20}$ Sul punto vedi l'accurata discussione in Musti, Demokratía, cit., cap. 4 .

${ }^{21}$ Per esempio, Luciano Canfora ha sostenuto che le sedute dell'assemblea fossero frequentate con regolarità soltanto dai membri dell'aristocrazia e dai teti, mentre contadini e artigiani erano scarsamente presenti. Vedi L. Canfora, La democrazia. Storia di un’ideologia, Laterza, Roma-Bari 2004, cap. 2; Id., Se la democrazia degli antichi sia ancora utile per emendare quella dei moderni, cit.

${ }^{22}$ Si tratta di una procedura, introdotta da Clistene, volta a decretare l'esilio decennale per un leader politico, spesso usata come strumento per l'eliminazione di un oppositore. La procedura cadde in disuso nel quarto secolo a.C. Vedi: Stockton, The Classic Athenian Democracy, cit., pp. 33-41; Thorley, Athenian Democracy, cit., pp. 44-45.

${ }^{23}$ Vedi Hansen, The Athenian Democracy in the Age of Demosthenes, cit., pp. $150-160$.

${ }^{24}$ Ivi, pp. 143-145. 
al pagamento di una multa di importo assai elevato e privato dei diritti civili e politici ${ }^{25}$.

- Boulé, consiglio dei cinquecento. Era composto da 500 membri di età superiore a 30 anni, 50 per ognuna delle 10 tribù. I membri venivano estratti a sorte (solo tra coloro che volevano essere eletti) per un anno e potevano ricoprire la carica solo due volte nella vita. In origine ne erano esclusi i teti. Svolgeva funzioni istruttorie rispetto all'ekklesía e ne predisponeva l'ordine del giorno ${ }^{26}$.

- Pritanía, commissione dei cinquanta. Era composta dai 50 membri della boulé afferenti alla medesima tribù - pritani. Restava in carica per 35-36 giorni. Organizzava i lavori della boulé. Era presieduta da un epístate, che variava ogni giorno. L'epístate era la somma carica dell'Atene democratica, a lui competevano incarichi di speciale importanza, come ricevere gli ambasciatori delle città vicine ${ }^{27}$.

- Magistrature: in assenza di un'amministrazione le funzioni di pubblica utilità venivano esercitate dai cittadini investiti del ruolo di magistrati. La gran maggioranza dei magistrati veniva sorteggiata (solo tra coloro che volevano essere eletti). Soltanto nei casi in cui fossero richieste competenze tecniche si procedeva a un'elezione. Le magistrature avevano una durata limitata nel tempo - generalmente un anno - e venivano esercitate collegialmente. $\mathrm{Al}$ termine del mandato, il magistrato doveva rendere conto del proprio operato e ogni cittadino poteva chiamarlo a rispondere di eventuali abusi davanti a un apposito tribunale ${ }^{28}$.

- Tribunali popolari: vi erano un gran numero di tribunali popolari con varie competenze. I giudici erano estratti a sorte fra i cittadini (solo tra coloro che volevano essere eletti). Le fasi istruttorie erano gestite da magistrati ${ }^{29}$.

${ }^{25}$ Ivi, pp. 205-212. Cfr. anche Cfr. M.H. Hansen, Graphe paranomon. La sovranità del tribunale popolare ad Atene nel IV secolo a. C. e l'azione pubblica contro proposte incostituzionali (ed. or. 1991), a cura di M.C. Rogozinski, Giappichelli, Torino 2001.

${ }^{26}$ Vedi: Stockton, The Classic Athenian Democracy, cit., pp. 84-95; Hansen, The Athenian Democracy in the Age of Demosthenes, cit., pp. 246-65; Thorley, Athenian Democracy, cit., pp. 28-32.

${ }_{27}$ Vedi: Hansen, The Athenian Democracy in the Age of Demosthenes, cit., p. 250; Thorley, Athenian Democracy, cit., p. 31; Musti, Demokratía, cit., pp. 140-145.

${ }^{28}$ Vedi: Stockton, The Classic Athenian Democracy, cit., pp. 105-116; Hansen, The Athenian Democracy in the Age of Demosthenes, cit., pp. 225-245; Thorley, Athenian Democracy, cit., pp. 40-43.

${ }^{29}$ Vedi: Stockton, The Classic Athenian Democracy, cit., pp. 96-103; Hansen, The Athenian Democracy in the Age of Demosthenes, cit., pp. 178-224; Thorley, Athenian Democracy, cit., pp. 35-38. 
- Nomothétai: come accennato più sopra l'assemblea non approvava leggi ma decreti. Le leggi, almeno dal quarto secolo a.C. erano competenza di speciali magistrati, i nomethétai appunto ${ }^{30}$.

Il quadro complessivo era tale che sussisteva un'alta probabilità che ciascun cittadino fosse coinvolto nell'amministrazione della cosa pubblica.

L'esperienza democratica dura poco meno di due secoli. Dopo la fioritura nel quinto secolo, in cui Pericle tiene le redini del governo della città dal 461 al 429 a.C., detenendo ripetutamente la carica di stratega ${ }^{31}$, le strutture democratiche subiscono una prima scossa con la sconfitta nella Guerra del Peloponneso e nel 322 a.C., in seguito alla conquista macedone, perdono ogni potere reale ${ }^{32}$.

Una breve notazione a proposito di Pericle. Pericle era alleato della potente famiglia degli Alcmeonidi, costruì la sua fortuna politica grazie all'appoggio della fazione popolare e si segnalò per l'adozione di misure 'populiste' - come la drastica limitazione dei poteri dell'Areopago ${ }^{33}$, vestigio del potere oligarchico, e l'introduzione di un gettone di presenza per la partecipazione ai lavori della boulé e dei tribunali. Quindi, è uno dei primi esempi di leader politico che forza le strutture democratiche grazie all'appoggio plebiscitario della parte popolare ${ }^{34}$.

\section{Le ragioni dell'estrazione a sorte}

Oggi può sembrare incongruo e 'antidemocratico' che gran parte delle cariche fosse assegnata per estrazione a sorte. La ragione essenziale di questa scelta, come ha argomentato il politologo Bernard Manin, può essere ricostruita come segue: l'estrazione a sorte realizzava nel modo più completo il principio cardine della democrazia ateniese, vale a dire il principio di rotazione delle cariche. L'azione dei magistrati era

${ }^{30}$ Vedi: Stockton, The Classic Athenian Democracy, cit., pp. 80-82; Hansen, The Athenian Democracy in the Age of Demosthenes, cit., pp. 161-177; Thorley, Athenian Democracy, cit., p. 60.

${ }^{31}$ Ogni anno venivano eletti - non sorteggiati - dieci strateghi, uno per ciascuna delle dieci tribù. Gli strateghi erano in sostanza generali con funzioni che si estendevano ben oltre la sfera prettamente militare. Vedi: Stockton, The Classic Athenian Democracy, cit., pp. 105-106; Hansen, The Athenian Democracy in the Age of Demosthenes, cit., pp. 268-270; Thorley, Athenian Democracy, cit., pp. 38-40.

${ }^{32}$ Sull'evoluzione della democrazia nell'età di Pericle e nei decenni successivi, cfr. Cartledge, Democracy, cit., capp. 5-13.

33 Più precisamente, la ridefinizione dei poteri dell'Areopago fu approvata dall'ekklesía nel 462 a.C. su proposta di Efialte - che morirà assassinato l'anno seguente - con il decisivo appoggio di Pericle.

${ }^{34}$ Sulla figura di Pericle la bibliografia è vastissima. Per tutti, si può vedere Mossé, Pericle. Linventore della democrazia, cit. 
controllata dall'assemblea cui essi dovevano rendere conto. Il fatto che le magistrature fossero estratte a sorte e avessero una durata limitata nel tempo assicurava una circolazione tra assemblea e magistrati. Sia i componenti dell'assemblea, sia i magistrati, sapendo che in breve volgere di tempo avrebbero potuto trovarsi a parti invertite, erano incentivati a operare correttamente: i magistrati non violando i propri doveri e i membri dell'assemblea non abusando del proprio potere di controllo 35 .

Inoltre, estrazione a sorte e rotazione delle cariche assicuravano un ampio coinvolgimento dei cittadini, che andava in parte a compensare la limitata partecipazione alle sedute dell'assemblea ${ }^{36}$. In questo senso, l'estrazione a sorte e la rotazione delle cariche rappresentavano un completamento dell'isegoría ${ }^{37}$. Al contrario, i meccanismi elettivi e la professionalizzazione della politica erano visti come un pericolo. I professionisti egemonizzano la politica, in quanto la libertà di eleggere comporta la libertà di rieleggere: contando sulle proprie qualità salienti cercano di monopolizzare l'agone politico, con ciò annullando di fatto l'uguaglianza fra i cittadini ${ }^{3}$.

\section{Esercito e dinamiche sociali}

Sin dagli albori della storia greca sussiste uno stretto legame tra l'organizzazione militare (e religiosa) della polis e la sua organizzazione politica. I re sono anche capi militari. Più tardi gli esponenti delle famiglie aristocratiche ricopriranno anche i ruoli di generali nelle campagne belliche. I cittadini sono gli uomini in armi. La corrispondenza tra organizzazione militare e organizzazione politica è ancora evidente nella riforma di Solone, laddove i cavalieri sono quei cittadini sufficientemente ricchi da poter disporre di un cavallo in battaglia, mentre gli zeugiti sono coloro che possedevano le ricchezze necessarie per coprire il costo dell'armatura e schierarsi come opliti (fanteria pesante). Non casualmente, il decisivo trasferimento di poteri dall'aristocratico Areopago all'ekklesía a prevalente composizione popolare avviene nel momento in cui il contributo dei teti come rematori sulle navi da guerra diventa indispensabile e quindi questa categoria, finora di fatto esclusa dalla vita politica, può reclamare un maggior coinvolgimento ${ }^{39}$. Su questo sfondo si innestano

35 Vedi Manin, Principî del governo rappresentativo, cit., pp. 29-48.

${ }^{36}$ Il nesso tra rotazione delle cariche e democrazia è analizzato in G. Cambiano, Come nave in tempesta. Il governo della città in Platone e Aristotele, Laterza, Roma-Bari 2016, cap. 1.

37 Vedi Manin, Principî del governo rappresentativo, cit., p. 48.

${ }^{38}$ Ivi, pp. 36-39.

39 Vedi Canfora, La democrazia, cit., pp. 34 sgg. 
le dinamiche sociali e i conflitti tra le famiglie più abbienti e la massa dei cittadini dotati di scarse risorse economiche ${ }^{40}$.

Anche da queste notazioni si vede come la democrazia ateniese nasca come metodo per organizzare la comunità, nel tentativo di costruire uno spazio politico esente dalla minaccia del conflitto interno, la stasis. Pertanto, è possibile giudicarla sotto il profilo della sua efficienza organizzativa. L'aspetto della valutazione 'ingegneristica' non va confuso con quello della valutazione assiologica. Oggi, infatti, noi riteniamo che il coinvolgimento di tutti nella gestione della cosa pubblica sia un valore e costituisca un diritto (in capo ai singoli soggetti). Platone e Aristotele giudicavano la democrazia essenzialmente dal punto di vista di una riflessione sui metodi più efficaci di gestire la polis in rapporto al conseguimento del bene comune. In quest'ottica non c'è da stupirsi se la democrazia fosse criticata in quanto realizzava un'inopportuna prevalenza della componente popolare ${ }^{41}$.

\section{Prime testimonianze sulla democrazia}

Il primo autore a discutere dei meriti e demeriti delle diverse forme di governo è stato lo storico Erodoto di Alicarnasso (490/480-430 ca. a.C.). Nel terzo libro della sua opera Le storie ${ }^{42}$, Erodoto inscena una discussione, che si sarebbe svolta in Persia, fra tre personaggi che presentano i rispettivi meriti delle tre principali forme di governo, governo del popolo, oligarchia e monarchia ${ }^{43}$. Il sostenitore dei meriti del governo popolare, Otane, ritiene che questa forma di governo sia preferibile in quanto evita la minaccia che il monarca abusi del proprio potere ${ }^{44}$.

Venendo più specificamente al dibattito sulla democrazia ateniese, una delle prima discussioni in merito è contenuta in uno scritto di ignoto autore, la Costituzione degli Ateniesi - conosciuta anche con il titolo La democrazia come violenza -, detta dello Pseudo-Senofonte perché

${ }^{40}$ Il nesso tra classi di censo e ruoli militari si affievolisce nel quarto secolo a.C. Vedi Hansen, The Athenian Democracy in the Age of Demosthenes, cit., pp. 108-109.

${ }^{41}$ Un libro importante che fa luce sul perché la quasi totalità degli autori classici esprima valutazioni critiche della democrazia è J.T. Roberts, Athens on Trial: The Antidemocratic Tradition in Western Thought, Princeton University Press, Princeton 1994.

${ }^{42}$ Vedi Erodoto, Le storie, lib. III, 80, 1-6.

43 Più propriamente, Erodoto fa riferimento all'isonomía, o al governo della maggioranza (plethos).

${ }_{44}$ Sull'interpretazione del passo erodoteo, cfr. G. Carillo, Nel molto c'è il tutto, la democrazia nel dibattito sui regimi politici (Erodoto, III, 8o, 1-6), in G. Duso (a cura di), Oltre la democrazia. Un itinerario attraverso i classici, Carocci, Roma 2004, pp. 31-53. 
tramandata nel corpus delle opere di Senofonte ${ }^{45}$. In questo breve trattato, che potrebbe risalire al periodo immediatamente successivo alla morte di Pericle, quindi alla fine del V secolo a.C., la democrazia viene descritta come il regime dove comanda la gente del popolo a danno delle famiglie nobili. L'autore, conosciuto come il 'Vecchio Oligarca', sostiene che questo stato di cose è una conseguenza del fatto che le fasce più basse della popolazione hanno guadagnato una sempre maggior importanza in seguito all'aumento della potenza militare navale di Atene. Grazie al governo democratico i poveri perseguono il proprio esclusivo interesse, sperperando il denaro pubblico e quello estorto alle città alleate di Atene.

Un'altra importante testimonianza sulla democrazia ateniese la troviamo nel discorso che lo storico Tucidide (460 ca.-398/396 a.C.) fa pronunciare a Pericle nella sua Storia della guerra del Peloponneso ${ }^{46}$. Nella ricostruzione di Tucidide, l'orazione funebre di Pericle per i caduti in battaglia ateniesi è costruita come un elogio della forma di governo della città. Pericle afferma infatti che il contrassegno della democrazia è dato dal perseguire il bene dei più, non quello di una ristretta minoranza e dal sostanziarsi in un assetto istituzionale nel quale vige l'uguaglianza di fronte alla legge e l'apertura delle cariche per i più meritevoli. Tuttavia, le parole di Pericle contengono anche elementi di ambiguità, elementi che hanno fatto sostenere a Luciano Canfora che il discorso di Pericle tradisce la consapevolezza di Tucidide dei pericoli insiti nel governo della maggioranza ${ }^{47}$.

\section{Platone}

Platone nacque ad Atene nel 428-427 a.C. e morì nel 348-347. Visse quindi in un periodo di decadenza della democrazia. Fra l'altro, fu un tribunale popolare democratico che condannò a morte ingiustamente nel 399 a.C. il suo maestro Socrate. Non c'è da meravigliarsi quindi che il suo giudizio sulla democrazia sia assai critico $^{48}$.

45 Vedi Anonimo Ateniese, La democrazia come violenza, a cura di L. Canfora, Sellerio, Palermo 1982.

${ }^{46}$ Vedi Tucidide, Storia della guerra del Peloponneso, lib. II, 37, 2.

47 Vedi Canfora, La democrazia, cit., cap. 1. Su Tucidide si possono vedere J. Ober, Political Dissent in Demochratic Athens: Intellectual Critics of Popular Rule, Princeton University Press, Princeton 1998, cap. 2; L. Canfora, Tucidide. La menzogna, la colpa, l'esilio, Laterza, Roma-Bari 2016.

${ }^{48}$ Gli studi più complete e aggiornati sul pensiero politico di Platone sono: G. Klosko, The Development of Plato's Political Theory, Oxford University Press, Oxford 2006; M. Schofield, Plato: Political Philosophy, Oxford University Press, Oxford 2006. Vedi, inoltre, C. Bobonich, Plato's Utopia Recast: His Later Ethics and Politics, Oxford University Press, Oxford 2004. In italiano, utili i saggi contenuti in M. Vegetti, Quindici lezioni su Platone, Einaudi, Torino 2003 e in Cambiano, Come 
Il dialogo platonico più famoso, la Repubblica ${ }^{49}$, presenta un'utopia della città perfetta, governata dall'elite filosofica, con una rigida divisione della popolazione in classi e un penetrante controllo della vita privata - che comporta l'abolizione della famiglia naturale e della proprietà. Questo ordinamento è visto da Platone come un rimedio alle tendenze degenerative che affliggevano l'Atene del suo tempo, in cui i detentori del potere blandivano le pulsioni acquisitive del popolo per riceverne l'appoggio. Esso mostra una discontinuità profonda rispetto all'organizzazione dell'Atene democratica, imperniata sul principio di rotazione delle cariche e sul sorteggio ${ }^{50}$. $\mathrm{Al}$ tempo stesso, promette di implementare un modello basato sulla natura. Infatti, dal momento che gli uomini non sono uguali per natura, si tratta di individuare un assetto che tenga conto delle differenze antropologiche tra gli individui, neutralizzando i pericoli che minacciano la sopravvivenza della polis, in primo luogo quello della spaccatura in gruppi distinti, in competizione fra loro, e instaurando un sistema nel quale la vocazione demagogica dei leader non trovi spazio ${ }^{51}$.

Platone mette così a fuoco la principale criticità del governo democratico: si tratta della costitutiva dipendenza dei leader dal favore della maggioranza, dipendenza che li porta ad assecondarne i desideri a scapito del perseguimento del bene comune. Per questo motivo si rende necessaria l'instaurazione di una forma di governo diversa, in cui la leadership sia assegnata a soggetti che possano governare senza dover dipendere da volontà e desideri esterni, concentrandosi unicamente sul bene dell'intera comunità. Ma affinché ciò sia possibile i governanti non dovranno essere distratti da interessi privati, pertanto non potranno possedere ricchezze ${ }^{2}$. Infatti, se essi si riappropriano dei beni materiali, le tendenze degenerative che affliggono la polis riaffiorano e si innesca una catena discendente di forme di governo corrotte che dall'ottima repubblica conduce alla timocrazia, all'oligarchia, alla democrazia - stigmatizzata in quanto governo dei poveri contro i ricchi, esito della libertà che diviene licenza -, fino alla tirannide ${ }^{53}$.

nave in tempesta, cit. Sulla critica di Platone alla democrazia, cfr.: Ober, Political Dissent in Demochratic Athens, cit., cap. 4; Th. Samaras, Plato on Democracy, Peter Lang, New York 2002.

49 Platone, La Repubblica, a cura di M. Vegetti, Bur, Milano 2007. Una valida introduzione al dialogo è J. Annas, An Introduction to Plato's Republic, Oxford University Press, Oxford 1981.

${ }^{5}$ Su questo punto, cfr. G. Cambiano, Platone e le tecniche, Laterza, Roma-Bari 1991, pp. 145-161.

${ }^{51}$ Vedi Platone, La Repubblica, cit., lib. IV, 422, e, 4-423, a, 5, p. 547. Cfr. anche Vegetti, Quindici lezioni su Platone, cit., lezione 6.

${ }^{52}$ Vedi Platone, La Repubblica, cit., lib. III, 416, d, 2-417, a, 5, pp. 533-535.

${ }^{53}$ Ivi, lib. VIII, pp. 927-1013. 
In un altro importante dialogo, il Politico, Platone sostiene che la politica è una scienza difficile che può essere padroneggiata solo da poche persone. L'uomo politico è simile al tessitore nel senso che deve saper intrecciare le diverse competenze necessarie per il governo della polis ${ }^{54}$. Pertanto, l'arte politica non può essere esercitata dalla massa incolta.

Platone introduce anche una tassonomia che sarà ripresa da Aristotele e distingue fra sei tipologie di forme di governo, tre rette e tre corrotte, segnate da violenza e illegalità, rispettivamente monarchia, aristocrazia e democrazia secondo le leggi, e tirannia, oligarchia e democrazia contra legem. Tra queste la democrazia è la peggiore delle forme rette - la migliore è la monarchia - e la migliore delle forme corrotte - la peggiore è la tirannia. Se ne deduce che la democrazia è scarsamente 'efficiente', nel bene come nel male ${ }^{55}$.

Per trovare una valutazione più positiva delle istituzioni democratiche dobbiamo andare a un dialogo tardo, le Leggi, probabilmente completato dall'allievo Filippo di Opunte dopo la morte del maestro. Qui Platone immagina una comunità ideale, avente tuttavia caratteristiche meno radicali di quella della Repubblica ${ }^{56}$ : il controllo sulla sfera privata è solo parziale, nel senso che viene ristabilita la famiglia naturale e la proprietà - le ricchezze individuali e le disuguaglianze, tuttavia, devono attestarsi entro certi limiti per evitare il rinascere delle conflittualità ${ }^{57}$. Inoltre, vengono recuperate le istituzioni democratiche ${ }^{58}$, attenuando l'idea, già esposta nel Politico, secondo la quale solo pochi sono competenti nell'arte di governo. L'elemento democratico viene sottolineato anche nel senso che qui Platone afferma, a differenza dei dialoghi precedenti, che sono due «le madri» di tutte le costituzioni esistenti: monarchia e democrazia, mentre le altre derivano da esse ${ }^{59}$.

L'elemento progettuale, che si ricollega all'eredità della Repubblica, è presente nella legislazione minuziosissima e in molti casi innovativa, volta alla completa regolazione delle condotte, che disciplina ambiti come l'educazione, il culto, l'organizzazione del lavoro, la vita sociale, il commercio, i crimini. Questa esplosione normativa segnala probabilmente la minor

${ }_{54}$ Platone, Politico, a cura di M. Migliori, Bompiani, Milano 2001, 311, b, 7-c, 7, p. 241.

55 Ivi, 302, b, 5-303, b, 7, pp. 207-209.

${ }^{56}$ Alcuni autori sostengono, infatti, che le Leggi rappresentano in una certa misura la ritrattazione della filosofia politica antidemocratica della Repubblica. Vedi: Samaras, Plato on Democracy, cit.; Bobonich, Plato's Utopia Recast, cit.

57 Vedi Platone, Leggi, a cura di F. Ferrari, Bur, Milano 2005, lib. V, pp. 389-453.

${ }_{58}^{8}$ Platone introduce una serie di istituzioni che ricordano quelle ateniesi: un'assemblea aperta a tutti i cittadini che portano o hanno portato le armi, un Consiglio di 360 membri che ne organizza i lavori, numerose magistrature, tribunali popolari. Vedi Platone, Leggi, cit., lib. VI, pp. 455-555.

${ }_{59}$ Ivi, lib. III, 693, d, 2-7, p. 293. 
fiducia dell'anziano Platone nella capacità del governante di assicurare la fioritura della polis. L'organo che ricorda più da vicino il collegio dei re-filosofi della Repubblica è il Consiglio notturno, un comitato di saggi cui è affidata l'unificazione dell'organizzazione politica ${ }^{60}$.

\section{Aristotele}

In sostanza, le critiche principale rivolte alla democrazia da Platone e dai suoi predecessori potrebbero essere compendiate in due punti: $a$ ) essere un regime 'disordinato', nel quale una parte prevale sulle altre e governa nel proprio esclusivo interesse; $b$ ) non poter garantire l'adeguata competenza da parte dei detentori del potere. Ora vedremo in che modo a queste critiche risponde Aristotele.

Aristotele nasce a Stagira, in Tracia, nel 384 a.C. Quindi è un meteco, uno straniero, non un cittadino di Atene. È allievo di Platone ad Atene. Dopo la morte del maestro si reca in Macedonia dove sarà precettore di Alessandro Magno. Muore nel 322 a.C. ${ }^{61}$

Con Aristotele si definisce il modello organicista di giustificazione del potere politico. Nel suo scritto principale di argomento politico, la Politica, Aristotele si propone di intraprendere una riflessione sul bene dell'uomo e sul modo di organizzare la vita comune in vista del suo perseguimento. Il punto di partenza della riflessione di Aristotele è l'idea secondo la quale l'uomo è un essere predisposto alla vita all'interno di una comunità: solo all'interno di una comunità l'uomo può conseguire il suo bene.

Vi sono tre tipologie fondamentali di comunità: la famiglia, il villaggio, la polis $^{62}$. Esse sono istituzioni naturali in quanto fondate su pulsioni - pulsione sessuale, desiderio di sopravvivenza - innate, quelle stesse pulsioni che Platone aveva cercato di imbrigliare nella Repubblica. I rapporti di potere che vigono nelle comunità si fondano e si giustificano a partire da differenze naturali, nello stesso modo in cui le differenze fra il padrone e gli schiavi, il marito e la moglie, il padre e i figli si radicano su diverse dotazioni di base. Inoltre, tali differenze definiscono un ordine gerarchico.

${ }^{60}$ Ivi, lib. XII, 961, a sgg., pp. 1067 sgg.

${ }^{61} \mathrm{Su}$ Aristotele politico, cfr.: F.D. Miller, Jr., Nature, Justice, and Rights in Aristotle's Politics, Oxford University Press, Oxford 1995; R. Kraut, Aristotle: Political Philosophy, Oxford University Press, Oxford 2002; D.J. Riesbeck, Aristotle on Political Community, Cambridge University Press, Cambridge 2016. In italiano cfr.: G. Bien, La filosofia politica di Aristotele (ed. or. 1973), il Mulino, Bologna 1985; Gf. Zanetti, La nozione di giustizia in Aristotele, il Mulino, Bologna 1993. Su Aristotele e la democrazia, cfr. C. Pacchiani, Democrazia e costituzione. La lezione di Aristotele, in Duso (a cura di), Oltre la democrazia, cit., pp. 55-76.

${ }^{62}$ Vedi Aristotele, Politica, commento di T.J. Saunders, R. Robinson, D. Keyt e R. Kraut, 2 voll., Fondazione Lorenzo Valla-Mondadori, Milano 2014-2015, lib. I, 1252, a, 24-1253, a, 39, vol. I, pp. 7-13. 
Un punto controverso della teoria aristotelica è la sua giustificazione della schiavitù. Al tempo di Aristotele la schiavitù era estremamente diffusa e nessuno prima di lui aveva avvertito la necessità di giustificarla. Aristotele ritiene che la schiavitù sia giustificata sulla base del deficit cognitivo degli schiavi, deficit che li rende inidonei a svolgere compiti più che esecutivi ${ }^{63}$. L'argomento di Aristotele tradisce un pregiudizio verso i lavori esclusivamente manuali e si correla con l'idea che la decadenza delle istituzioni politiche ateniesi sia dipesa dalla massiccia urbanizzazione e dall'ingresso in politica di masse incolte distanti dalla figura archetipica del piccolo proprietario terriero ${ }^{64}$.

La polis viene vista come prosecuzione naturale della famiglia. Tuttavia, la polis è anche anteriore alla famiglia e agli individui ${ }^{65}$ : rappresenta il Tutto a partire dal quale è possibile comprendere il ruolo delle parti e coordina in un certo senso i rapporti di dominio sottostanti. L'orientamento complessivo è comunitario. In questo assetto nessuna famiglia, né alcun individuo è più importante degli altri. Nel modello organicistico l'ambito dei rapporti privati anteriori alla costituzione del potere politico non va visto in opposizione a esso ma come una sua precondizione. Ne risulta che l'impostazione aristotelica è segnata da un marcato contrasto con la tradizione moderna, in cui il potere dello Stato sarà derivato dal potere naturale degli individui.

Aristotele critica la concezione platonica della famiglia e della proprietà sostenendo l'implausibilità del modello della Repubblica ${ }^{66}$. Questa critica si innesta su un atteggiamento di fondo: Aristotele, a differenza di Platone, non ritiene che lo scopo della filosofia sia quello di progettare dalle fondamenta un modello ideale di comunità. Dal momento che la comunità politica rappresenta una prosecuzione della famiglia e del villaggio, la polis non può che conservare gli assetti e le forme di potere preesistenti. L'indagine filosofico-politica ha piuttosto come scopo quello di elaborare una serie di precetti rivolti al legislatore che consentano a quest'ultimo di rettificare l'assetto politico esistente ${ }^{67}$.

L'atteggiamento 'anti-rivoluzionario' di Aristotele comporta anche una valutazione molto più favorevole del metodo democratico, che lo Stagirita riconosce come centrale nell'esperienza storica di Atene. Questa valutazione si lega all'idea che le tipologie di potere presentate nel primo Libro - potere del padrone sugli schiavi, del marito sulla moglie, del padre sui figli - si differenzino dal potere del governante sui governati, dal

${ }_{3}^{6}$ Ivi, lib. I, 1253, b, 23-1255, a, 2, vol. I, pp. 15-21. Sul tema, cfr. Cambiano, Come nave in tempesta, cit., cap. 8.

${ }^{64}$ Vedi Aristotele, Politica, cit., lib. VI, 1318, b, 6-1319, b, 32, vol. II, pp. 95-103.

${ }_{65}$ Ivi, lib. I, 1253, a, 20-29, vol. I, p. 11.

${ }^{66}$ Ivi, lib. II, pp. 55-127.

${ }^{67}$ Cfr. Pacchiani, Democrazia e costituzione, cit., pp. 60-61. 
momento che quest'ultimo si esercita su individui liberi e quindi non è giustificabile a partire da differenze naturali ${ }^{68}$. Ne deriva che il potere dei governanti sui governati debba prevedere una rotazione delle cariche ${ }^{69}$. Ma ciò comporta che le capacità di governo non siano appannaggio solo di poche persone, poiché la rotazione delle cariche presuppone la sovranità dell'assemblea che le elegge e che conserva la competenza sulle questioni più importanti ${ }^{70}$.

Aristotele, quindi, argomenta in favore della capacità di governare da parte di una molteplicità di individui: in contrasto con la tesi platonica del Politico, sostiene che un vasto insieme di persone, anche se queste non dispongono singolarmente delle competenze richieste, può presentare le capacità necessarie per governare con successo ${ }^{71}$.

Nel III libro della Politica Aristotele recupera la tassonomia platonica delle forme di governo esposta nel Politico. Dunque, ci sono sei forme di governo, tre rette - monarchia, aristocrazia e politeia, secondo che a governare sia uno solo, pochi o molti - e tre corrotte - tirannia, oligarchia e democrazia. La differenza sostanziale tra le forme rette e quelle corrotte risiede nel fatto che, mentre nelle prime il potere è esercitato nell'interesse comune, nelle seconde viene esercitato nell'interesse dei soli governanti ${ }^{72}$.

Aristotele si discosta, però, da Platone nel modo in cui istituisce la gerarchia fra le diverse tipologie politiche. Ritenendo preferibile il governo della legge al governo degli uomini - giacché la legge, a differenza degli uomini, è impermeabile alle passioni ${ }^{73}$ - Aristotele non può condividere il giudizio platonico che pone al vertice delle forme politiche la monarchia ${ }^{74}$. La forma migliore di governo consiste piuttosto in un regime misto, una commistione tra il governo di pochi e quello di molti, e si può realizzare soltanto nelle città in cui sia più ampio il ceto medio ${ }^{75}$. Tale regime prevedrà bassi requisiti censitari per la partecipazione alle assemblee $\mathrm{e}$ l'elezione - non il sorteggio - dei più meritevoli alle magistrature ${ }^{76}$. Dunque, anche il governo dei molti può realizzare una forma politica ordinata

${ }^{68}$ Vedi Aristotele, Politica, cit., lib. I, 1255, b, 16-20, vol. I, p. 25.

${ }^{69}$ Ivi, lib. III, 1279, a, 7-8, vol. I, p. 151. Sul tema della rotazione delle cariche in Aristotele, cfr. anche Cambiano, Come nave in tempesta, cit., cap. 4.

${ }^{70}$ Cfr. Cambiano, Come nave in tempesta, cit., pp. 72-73.

${ }^{71}$ Vedi Aristotele, Politica, cit., lib. III, 1281, a, 40 e sgg., vol. I, pp. $163 \mathrm{sgg}$.

${ }^{72}$ Ivi, lib. III, 1279, a, 17-21, vol. I, p. 151.

73 Ivi, lib. III, 1286, a, 16-20, vol. I, p. 187.

74 Platone - nel Politico, cit., 294, a, 1-c, 4, pp. 181-183 - sosteneva che il governo dell'uomo saggio fosse l'alternativa preferibile, perché anche la legge più accurata non può tenere conto delle variabili contestuali che si presentano nei casi concreti. Questo atteggiamento viene, tuttavia, sensibilmente attenuato nelle Leggi.

75 Vedi Aristotele, Politica, cit., lib. IV, 1295, a, 25-1296, b, 12, vol. I, pp. 239-245.

${ }^{76}$ Ivi, lib. IV, 1294, a, 30-1294, b, 41, vol. I, pp. 233-237. 
ed efficiente, a patto che vengano rispettate alcune condizioni attinenti soprattutto all'educazione dei cittadini ${ }^{77}$.

In definitiva, Aristotele può essere riconosciuto come il vero fondatore della teoria democratica, in quanto sostiene, contro Platone, la possibilità di un governo dei molti capace di conseguire il bene comune. Al tempo stesso, precisa che non ogni moltitudine può esprimere le competenze necessarie per governare ${ }^{78}$, pertanto, la possibilità di un governo democratico efficiente è legata alla preventiva selezione dell'insieme di individui che deve prendere le decisioni più rilevanti. Per questo motivo, la politeia è compatibile con la teorizzazione aristotelica della schiavitù: perché lo schiavo è segnato da una disuguaglianza naturale, che incide sul suo status politico.

Infine, è appena il caso di ricordare che Aristotele, insieme ai suoi allievi, si dedicò nell'ultimo periodo della sua attività a una vasta raccolta degli ordinamenti delle città greche. Di essa ci è pervenuto un breve scritto che ripercorre la storia e descrive le istituzioni democratiche di Atene, la Costituzione degli Ateniesi ${ }^{79}$.

77 Ivi, libb. VII-VIII, vol. II, pp. 121-221.

${ }^{78}$ Altrimenti, anche un branco di animali potrebbe farlo. Vedi Aristotele, Politica, cit., lib. III, 1281, b, 17-22, vol. I, pp. 163-165.

79 Aristotele, Costituzione degli ateniesi, a cura di P.J. Rhodes, Fondazione Lorenzo Valla-Mondadori, Milano 2016. 



\section{Capitolo secondo \\ Democrazia e modernità}

\section{Il modello romano}

Dopo la fine della democrazia ateniese il modello di governo democratico conosce una lunga eclissi che si protrarrà fino alle soglie della modernità. Prima di congedarci dall'esperienza politica di Atene può essere però utile riassumerne alcuni punti salienti.

Come si è visto il modello democratico ateniese presentava alcune caratteristiche ben definite: $a$ ) i criteri di definizione della cittadinanza erano molto esigenti - soprattutto per quanto riguarda la doppia discendenza ateniese. Ne derivava una limitata inclusività delle istituzioni democratiche; $b$ ) d'altra parte, tutti i cittadini erano uguali quanto alla partecipazione all'assemblea, senza diversificazioni censitarie; c) i cittadini partecipavano direttamente alla gestione della cosa pubblica. Vale la pena di notare che $a$ ), $b$ ) e c) sono strettamente collegate fra loro, nel senso che la possibilità di una partecipazione egualitaria, che si estrinsecava nell'inclusione nell'assemblea e nella rotazione delle cariche, era resa possibile da una preventiva selezione di coloro che erano veramente uguali fra loro. Per esempio, come abbiamo visto, il modello aristotelico era basato non solo sull'esclusione di donne e schiavi ma anche sulla marginalizzazione dei lavoratori manuali.

Caratteristiche analoghe, peraltro, non si ritrovano a Roma nel periodo repubblicano. L'elemento di cui soprattutto avvertiamo la mancanza - e che sembra essenziale per poter parlare di democrazia - è quello di cui al punto $b$ ), l'uguaglianza fra tutti i cittadini quanto alla capacità di incidere sulle decisioni politiche. La partecipazione alle assemblee avveniva, infatti, sulla base di classificazioni di tipo censitario: questo vale soprattutto per l'assemblea più importante, i comizi centuriati, che eleggeva le magistrature più importanti, consoli, censori e pretori, 
e dichiarava la guerra. In questa assemblea i cittadini votavano per centurie, vale a dire secondo classificazioni di origine militare basate sul censo - dal momento che coloro che erano più ricchi erano anche quelli che contribuivano in misura maggiore alle spese militari. Nei comizi centuriati i cittadini più abbienti erano raccolti in 98 centurie, più del resto della cittadinanza ${ }^{1}$, anche se in termini numerici rappresentavano una ristretta minoranza.

L'elemento di riequilibrio era rappresentato, da un lato, dai comizi tributi - l'assemblea che approvava la maggior parte delle leggi, nella quale si votava per tribù e in cui quindi i più ricchi non disponevano della maggioranza -, e, dall'altro, dai tribuni della plebe - magistrati che avevano il compito di evitare che si abusasse del potere politico in danno della parte popolare ${ }^{2}$.

Per queste ragioni, si può dire che la repubblica romana non era organizzata secondo criteri democratici. Il modello politico romano era piuttosto riconducibile, come ebbe a scrivere lo storico greco del II secolo a.C. Polibio nel sesto libro delle Storie ${ }^{3}$, alla forma del governo misto. Tipica del governo misto è, infatti, la compresenza di elementi monarchici - in primo luogo, i consoli, i magistrati più importanti, che comandavano l'esercito in guerra -, aristocratici - il ruolo del senato, detentore dell'auctoritas, da cui promanava la stessa potestas dei magistrati, controllato dalle famiglie più eminenti - e democratici - le istituzioni assembleari, in particolare, i comizi tributi.

\section{Modelli di giustificazione del potere nel Medioevo}

Durante il Medioevo il modello democratico non trova applicazioni, almeno fino all'anno Mille. L'affermazione del Cristianesimo conduce a un profondo ripensamento delle categorie politiche. Fino alla diffusione degli scritti di Aristotele nel XIII secolo, si eclissa l'approccio scientifico alla politica che proprio con Aristotele si era affermato. Prevale piuttosto l'idea secondo la quale il potere deriva dall'alto, discendendo al sovrano da Dio, è reso necessario dal peccato originale ed è preordinato alla salvezza spirituale.

Questa metafisica discendente corrisponde a una concezione della società come corpo vivente, ordinato da precise gerarchie che assegnano

${ }^{1}$ Questi assetti si modificano nella seconda metà del III secolo a.C. in senso più favorevole alle classi popolari.

${ }^{2}$ Cfr.: L.R. Taylor, Roman Voting Assemblies: From the Hannibalic War to the Dictatorship of Caesar, The University of Michigan Press, Ann Arbour 1966; A. Lintott, The Constitution of the Roman Republic, Oxford University Press, Oxford 2003.

${ }_{3}$ L'opinione di Polibio fu ripresa da Cicerone nel De re publica e successivamente da Nicolò Machiavelli nei Discorsi sulla prima deca di Tito Livio. 
a ciascun individuo il proprio posto e la propria funzione entro un disegno complessivo - di cui l'ordine politico è parte - tendente al bene comune ${ }^{4}$. D'altra parte, la strutturazione gerarchica della società e la stessa concezione complessiva del cosmo non costituiscono un'invenzione medievale, ma risentono in modo determinante dell'eredità di Roma, cristianizzata nel quarto secolo d.C. 5

Nel corso dell'Alto Medioevo il modello discendente dà corpo al dualismo tra potere del pontefice e potere dell'imperatore, e si coniuga, secondo i canoni dell'agostinismo politico - da Agostino di Ippona (354-430) -, alla cosiddetta teoria delle due spade, secondo la quale l'auctoritas del papa, erede di quella dell'Impero romano, si confronta con la potestas secolare, che però rimane alla prima gerarchicamente subordinata. Fino al XII secolo, prevale una visione della politica di stampo agostiniano, incentrata sulla tesi della superiorità del potere spirituale, nell'ambito di una concezione tipicamente religiosa dello spazio politico. In questa concezione il suddito è assimilato a un viator, incapace di provvedere a sé stesso in conseguenza del peccato originale e bisognoso della guida della legge di cui il sovrano è custode.

La situazione inizia però a mutare dopo l'anno Mille, in un quadro di importanti trasformazioni politiche e sociali, segnate dalla crescente contrapposizione tra il potere spirituale e quello temporale, che conducono al graduale superamento dell'ottica agostiniana. Si aprono così gli spazi per una considerazione affatto inedita del ruolo dei singoli all'interno delle strutture politiche. Mentre in epoca altomedievale i sudditi non avevano alcuna parte nel meccanismo di giustificazione del potere, che dipendeva unicamente dal rapporto che il sovrano intratteneva con la Legge, adesso la giustificazione dell'operato del sovrano inizia a essere correlata al consenso dei sudditi.

Questo ribaltamento di prospettiva è indubbiamente collegato al tentativo dell'imperatore di contrapporsi all'auctoritas del papa, come testimonia il dibattito, che impegna i giuristi filoimperiali a partire dall'XI secolo, intorno all'interpretazione di alcune massime contenute nel Corpus Iuris Civilis, la grande raccolta di diritto romano voluta dall'Imperatore bizantino Giustiniano I. A questo proposito, si può ricordare la cosiddetta Lex regia de imperio, vale a dire la norma secondo la quale la legittimazione dell'esercizio del potere supremo va cercata in un preciso atto di delega operato dal popolo in favore del governante ${ }^{6}$.

${ }_{4}$ Cfr. W. Ullmann, Il pensiero politico nel Medioevo (ed. or. 1965), Laterza, Roma-Bari 1989.

${ }^{5}$ Cfr. J. Coleman, A History of Political Thought: From the Middle Ages to the Renaissance, Blackwell, Oxford 2000.

${ }^{6}$ I giuristi peraltro si dividevano fra coloro che, come Irnerio, consideravano il trasferimento di potere dal popolo al re come definitivo e irrevocabile, e coloro 
Più in generale, la riscoperta del diritto romano, che inizia sempre nell'XI secolo, segna uno snodo fondamentale nella costruzione delle categorie politiche moderne. Un'altra massima che merita di essere ricordata in questa sede recita: «Quod omnes tangit, ab omnibus tractari et approbari debet». L'interpretazione di questo principio impegnerà i giuristi, inizialmente nell'ambito del diritto canonico, a partire dal XII secolo. Adattando questa massima, proveniente dal diritto privato, al contesto pubblicistico, si ottiene l'effetto di far dipendere la fondazione del potere del sovrano dal consenso dei soggetti interessati. Si stabilisce così un basilare principio democratico: il potere è originariamente in mano del popolo che deve approvare le scelte politiche più importanti, per esempio, in materia di imposizione fiscale, secondo una prassi che evolverà verso la costituzione delle moderne assemblee rappresentative ${ }^{7}$.

Un altro passaggio cruciale si verifica nel XIII secolo con la traduzione in latino di Guglielmo di Moerbecke della Politica aristotelica. La 'riscoperta' di Aristotele segna uno snodo fondamentale del pensiero politico in quanto nell'opera del filosofo greco i pensatori medievali trovano le categorie concettuali per impostare un nuovo tipo di riflessione. Il linguaggio aristotelico si aggiunge, nella costellazione concettuale dei pensatori medievali, all'idioma biblico, a quello classico, al lessico dei giuristi e dei canonisti, predisponendo gli strumenti necessari per tematizzare problematiche nuove rispetto al passato ${ }^{8}$. Attraverso il confronto con la tesi aristotelica secondo la quale l'uomo è un animale sociale e le istituzioni politiche 'prodotti' naturali, gli autori medievali si pongono la questione di quale sia la forma di governo preferibile, fino ad allora implicitamente risolta nel senso di ritenere che la forma di governo monarchica sia la migliore. Per questo tramite, si realizza una sorta di fusione fra tra il modello discendente della legittimazione del potere e l'organicismo aristotelico. Sarebbe probabilmente fuorviante, tuttavia, leggere queste trasformazioni in senso democratico o proto-democratico, dal momento che il ruolo del consenso popolare non è mai disgiunto da una concezione complessiva che prevede la compresenza di diverse tipologie e diverse fonti di legittimazione del potere, secondo il modello classico, romano e aristotelico al tempo stesso, del governo misto.

che, come Azzone, sottolineavano che il popolo potesse riappropriarsi del potere ceduto al principe. Cfr. M. Conetti, Il pensiero politico dei giuristi, in M. Fumagalli Beonio Brocchieri et al., Il pensiero politico medievale, Laterza, Roma-Bari 2000.

7 Per una rassegna storica delle fortune del principio «Quod omnes tangit», vedi S. Cassese, Il diritto globale. Giustizia e democrazia oltre lo Stato, Einaudi, Torino 2009, pp. 157 sgg. Cfr. inoltre: Coleman, A History of Political Thought, cit., pp. 42-46; M. Loughlin, Foundations of Public Law, Oxford University Press, Oxford 2010, cap. 1.

${ }^{8}$ Sul tema dei diversi linguaggi del pensiero politico, cfr. A. Black, Political Thought in Europe 1250-1450, Cambridge University Press, Cambridge 1992. 
Il pensatore nel quale i diversi motivi che animano la riflessione politica medievale trovano una sintesi originale è Tommaso d'Aquino (12251274). Per Tommaso il tema della naturale socialità dell'essere umano tema che l'Aquinate eredita da Aristotele - si lega a quello dell'esistenza di una Legge naturale che discende all'uomo da Dio. L'esito è una concezione secondo la quale l'istituzione del potere politico non deve porre rimedio alla Caduta e al peccato ma è una naturale conseguenza della necessità di organizzare la vita associata ed è diretta al perseguimento del bene comune. Ciò non significa che nella riflessione politica dell'Aquinate gli individui non abbiamo alcun rilievo. Infatti, nella Summa Theologiae Tommaso seguendo Aristotele sembra ritenere preferibile una forma di governo mista, nella quale il sovrano sia affiancato da un corpo di cittadini qualificati scelti da - e fra - il popolo9.

\section{La partecipazione popolare nei comuni}

Alla vicenda che conduce, attraverso il superamento dell'agostinismo politico e la riscoperta di Aristotele, a legare la giustificazione del potere alla volontà del popolo si affianca una diversa esperienza, tramite la quale si impone il principio della partecipazione popolare alla gestione del potere. A partire dall'XI secolo si vanno affermando, soprattutto nell'Italia centro-settentrionale e nel Nord Europa, nuovi soggetti politici, che rivendicano autonomia rispetto al Papato e all'Impero. Si tratta delle città.

Lo strumento che sancisce questa autonomia è la costituzione di una coniuratio, vale a dire di una sorta di associazione sancita da un giuramento fra $\mathrm{i}$ cittadini, diretta a creare una comunità politica indipendente dall'ordine feudale, un comune appunto ${ }^{10}$. In un primo tempo questi organismi rimangono saldamente nelle mani delle famiglie aristocratiche. Secondo un modello abituale, coloro che dispongono delle risorse economiche necessarie per sopportare le spese militari, egemonizzano la vita politica. In seguito, a partire dal XIII e XIV, secolo si generano dinamiche conflittuali che contrappongono gli esponenti delle famiglie più illustri alla parte popolare. Si definisce così un complesso sistema istituzionale, saldamente radicato nel modello di derivazione romanistica del governo misto, basato su assemblee diversificate - varie tipologie di consigli più ristretti, oltre all'assemblea cittadina, detta Arengo o

9 Vedi Tommaso d'Aquino, Summa Theologiae, $\mathrm{I}^{\mathrm{a}}-\mathrm{II}^{\mathrm{ae}}$, q. 105, a.1. Sul pensiero politico di Tommaso d'Aquino, cfr. J. Finnis, Aquinas: Moral, Political, and Legal Theory, Oxford University Press, Oxford 1998.

${ }^{10}$ Su questi temi, cfr.: D. Waley, T. Dean, The Italian City Republics, Routledge, Abingdon 2009; M. Ascheri, Le città-Stato. Le radici del municipalismo e del repubblicanesimo italiano, il Mulino, Bologna 2006. 
Parlamento - e magistrature elettive - in un primo tempo il comune è governato da due consoli eletti dall'assemblea, in seguito da un podestà, cioè da un magistrato proveniente da un'altra città e per questo motivo presumibilmente imparziale rispetto ai conflitti interni ${ }^{11}$.

Nell'ambito di questo quadro istituzionale si sviluppa un sistema che prevede un'ampia partecipazione politica nel segno dell'uguaglianza di tutti i cittadini di fronte alla legge. Questo elemento è importante, perché permette di enucleare un secondo senso in cui si fa strada la rilevanza politica del popolo. Nella sezione precedente si è visto come, a partire dall'XI secolo, acquista visibilità il principio secondo il quale la legittimazione del sovrano riposa sul consenso popolare. Si delinea in questo modo un vettore che potremmo dire 'verticale' di rilevanza: il sovrano esercita un potere che gli deriva, in ultima analisi, dal popolo. L'esperienza dei comuni, e poi delle città-Stato ci propone invece un modello 'orizzontale', in cui il popolo, nell'estensione delle sue diverse componenti, si afferma come detentore e gestore del potere sovrano.

Tuttavia, questa affermazione richiede alcune cautele: da un lato, il controllo delle dinamiche politiche resta saldamente appannaggio di élite ristrette mentre gli strati più bassi della popolazione restano esclusi dalla gestione della cosa pubblica; dall'altro, la partecipazione è sempre mediata dall'appartenenza a organismi di raccordo, in primo luogo le corporazioni che raccolgono i lavoratori di un certo settore. I due aspetti sono ovviamente collegati fra di loro, perché le corporazioni più prestigiose - in primo luogo, quelle dei banchieri e dei mercanti - godono di un maggior peso politico e, d'altra parte, i lavoratori non specializzati, non essendo iscritti ad alcuna corporazione, non partecipano affatto alla vita politica. Ciò significa che la dimensione popolare della sovranità presuppone l'operatività di meccanismi di esclusione, che strutturano la moltitudine, altrimenti informe, del corpo dei cittadini.

Questo tipo di dinamiche conducono alle rivolte popolari che hanno luogo in varie parti d'Europa nella seconda metà del XIV secolo: per esempio, a Firenze nel 1378 ha luogo il Tumulto dei Ciompi - nome che designava i lavoratori salariati dell'Arte della Lana, che chiedevano di costituire una propria corporazione. Questo episodio si inscrive in una dialettica di conflitti tra le famiglie più illustri e la parte popolare, dialettica che si risolverà, a metà del secolo successivo, con l'ascesa al potere, grazie all'appoggio proprio della parte popolare, della famiglia dei Medici. Ai Medici succederà una nuova fase repubblicana, fino all'instaurazione, nel 1569, del Granducato di Toscana. Il tasso di democraticità di queste istituzioni resterà comunque molto basso, se è vero che nella

${ }^{11}$ Vedi Waley, Dean, The Italian City Republics, cit., pp. 34-44. 
Firenze repubblicana del primo Cinquecento partecipavano alle sedute del Consiglio maggiore circa 3.000 cittadini $^{12}$.

\section{Teorizzare l'autonomia}

La riscoperta della Politica di Aristotele segna profondamente il dibattito bassomedievale, lasciando tracce tanto negli scritti degli autori che sostengono che il potere dell'imperatore sia subordinato a quello del papa, come Egidio Romano (1242-1316) - in cui prevale, tuttavia, un'ispirazione agostiniana -, quanto, soprattutto, in coloro che sostengono le ragioni dell'Impero, come Giovanni da Parigi (1250-1306) e Dante Alighieri (1265-1321) $)^{13}$.

Ma la vera novità della seconda metà del XIII secolo è rappresentata dal fatto che l'ascesa dei comuni come nuovi soggetti politici capaci di rivendicare autonomia sia rispetto all'imperatore che al papa viene ratificata a livello teorico. Ciò avviene sia nell'ambito più propriamente politico, sia in quello giuridico. Sul primo versante, merita qui ricordare l'opera di autori come Brunetto Latini (1220 ca.-1294) e Tolomeo da Lucca (1236 ca.-1327), allievo di Tommaso d'Aquino, che, contrapponendo la forma di governo monarchica a quella 'politica', basata sul consenso popolare e la sottoposizione alla legge, giustificano l'autogoverno delle città.

Il contributo più significativo alla giustificazione dell'autonomia delle città e l'elaborazione teorica più articolata dell'idea che il fondamento del potere risiede nel consenso del popolo si trova però in Marsilio da Padova (1285-1343). Marsilio, nella sua opera maggiore, il Defensor pacis $^{14}$, compie un passo decisivo verso la desacralizzazione del potere, volgendo definitivamente le spalle all'agostinismo politico e contrapponendosi recisamente ai teorici ierocratici. D'altra parte, la proposta

${ }^{12}$ Cfr. N. Rubinstein, Studies in Italian History in the Middle Age and Renaissance, vol. I, Political Thought and the Language of Politics, Edizioni di Storia e Letteratura, Roma 2004. Sulla storia di Firenze, si può vedere G.A. Brucker, Dal Comune alla Signoria. La vita pubblica a Firenze nel primo Rinascimento (ed. or. 1977), il Mulino, Bologna 1981.

${ }_{13}$ Un'eccezione, in questo senso, è rappresentata da Guglielmo da Ockham (1285 ca.-1347), nella cui opera politica, che rappresenta una strenua difesa del potere dell'imperatore, l'influenza aristotelica è scarsamente ravvisabile. In tema, mi sia consentito rinviare a L. Marchettoni, Ockham e l'origine dei diritti soggettivi, «Quaderni fiorentini per la storia del pensiero giuridico moderno», 37, 2008, pp. 19-64.

${ }^{14}$ Su Marsilio, vedi: V. Omaggio, Marsilio da Padova. Diritto e politica nel Defensor Pacis, Editoriale Scientifica, Napoli 1995; M. Merlo, Marsilio da Padova. La politica come grammatica del mutamento, FrancoAngeli, Milano 2003; G. Briguglia, Marsilio da Padova, Carocci, Roma 2013. 
di Marsilio non si risolve nemmeno in una unilaterale rivendicazione della supremazia imperiale.

In un contesto profondamente segnato dall'eredità aristotelica - evidente, per esempio nella discussione intorno alle diverse forme di governo o nella riabilitazione della razionalità politica dei cittadini -, Marsilio sostiene, infatti, che la funzione di governo deve essere esercitata da un magistrato eletto, e deve essere orientata all'implementazione di una legge positiva, distinta dalla legge naturale. Tale legge, poi, deve essere approvata dalla totalità dei cittadini o dalla sua valentior pars ${ }^{15}$, dove questa espressione, su cui si sono affaticate generazioni di interpreti, potrebbe essere letta, valorizzando l'aristotelismo di Marsilio, nel senso di riconoscere che l'attitudine a perseguire il bene comune non può essere attribuita a qualsiasi insieme di individui ma solo a 'porzioni qualificate' di tutto il popolo. Quindi, anche la proposta marsiliana mette capo a un regime misto, in cui il consenso popolare funge da contrappeso rispetto al detentore del potere di governo.

Per quanto riguarda l'apporto dei giuristi, dopo la fase iniziale, che aveva visto la convergenza tra i progetti politici imperiali e le dottrine dei Glossatori - così erano detti i giuristi impegnati nell'opera di interpretazione del Corpus Iuris Civilis, da glossa: nota esplicativa -, nella contesa fra Impero e Papato, si apre un nuovo periodo nel quale i giuristi appartenenti alla scuola dei Commentatori, come Bartolo da Sassoferrato (1314-1357), rileggono il diritto romano in senso favorevole all'autonomia dei comuni avverso le prerogative imperiali, adottando un atteggiamento ermeneutico meno vincolato alla lettera del testo e quindi più creativo. Viene così teorizzata, da un lato, l'esistenza di precisi limiti al potere sovrano, come i diritti di proprietà individuali, garantiti dalle norme del diritto naturale, dall'altro, la compresenza di diversi soggetti dotati di sovranità e vincolati al perseguimento del bene comune ${ }^{16}$.

\section{Il recupero della tradizione repubblicana}

Nel secolo successivo, a Firenze, come hanno messo in luce i classici studi di John Pocock e Quentin Skinner ${ }^{17}$, la riscoperta della tradizione repubblicana romana conduce a una nuova considerazione delle virtù civiche. La lettura di autori come Sallustio, Cicerone e Tito Livio condu-

${ }^{15}$ Vedi Marsilio da Padova, Il difensore della pace, a cura di C. Vasoli, Utet, Torino 196o, lib. I, XII, 3, p. 169.

${ }^{16}$ Vedi Conetti, Il pensiero politico dei giuristi, cit.

${ }_{17}$ J.G.A. Pocock, Il momento machiavelliano. Il pensiero politico fiorentino e la tradizione repubblicana anglosassone (ed. or. 1975), il Mulino, Bologna 1980; Q. Skinner, Le origini del pensiero politico moderno (ed. or. 1978), 2 voll., il Mulino, Bologna 1989. 
ce a un maggiore apprezzamento della partecipazione dei cittadini alla cosa pubblica, del valore della libertà e dell'uguaglianza, dell'importanza dell'autogoverno e dell'indipendenza. D'altra parte, è evidente anche la continuità con la riflessione precedente, in particolare con le coordinate teoriche della proposta marsiliana e con le acquisizioni dei giuristi della scuola dei Commentatori ${ }^{18}$.

Questi temi sono centrali negli scritti dei due più importanti Cancellieri della repubblica fiorentina, Coluccio Salutati (1331-1406) e Leonardo Bruni (1370-1444). Quest'ultimo, nella sua opera maggiore, l'Historiarum Florentini populi, libri XII, traccerà un esplicito parallelismo tra Firenze e l'Atene di Pericle, sostenendo l'esistenza di un legame tra splendore artistico, prosperità economica e libertà politica.

La vis repubblicana tende però a ripiegarsi su sé stessa nel corso del XV secolo, in coincidenza con l'ascesa a Firenze della famiglia dei Medici e, più in generale, con la diffusione in Italia di Signorie e Principati. Questa trasformazione è particolarmente notevole, in quanto segna il passaggio dai governi elettivi ai governi ereditari. Questo ripiegamento è evidente, per esempio, nei Libri della famiglia di Leon Battista Alberti (1404-1472) e nella coeva fioritura della trattatistica sull'ottimo principe.

L'intera vicenda repubblicana viene poi ripensata, all'interno di una trattazione particolarmente originale e influente, nelle opere di Nicolò Machiavelli (1469-1527) ${ }^{19}$. Machiavelli, nelle sue opere politiche maggiori, soprattutto nei Discorsi sopra la prima Deca di Tito Livio e ne Il Principe $^{20}$, ripercorre la lezione dei classici adattandola alle vicende contemporanee. Lo studio della storia mostra soprattutto una fondamentale instabilità politica, che motiva una continua alternanza tra monarchia, aristocrazia e democrazia. Ciò comporta, da un lato, che non esista una forma di governo pura migliore delle altre, dall'altro che le più favorevoli prospettive di stabilità e progresso possono essere offerte da forme miste, nelle quali diversi soggetti e diversi interessi possano essere ugualmente coinvolti. Il vantaggio delle forme di governo miste, infatti, è quello di fornire un assetto istituzionale nel quale le dinamiche conflittuali, che divampano sempre all'interno della società, vengono incanalate in modo da sprigionare energie positive.

${ }^{18}$ Questo punto è stato sottolineato soprattutto da Skinner, che accostava Marsilio a Bartolo da Sassoferrato. Vedi Skinner, Le origini del pensiero politico moderno, cit., vol. I, p. 139.

19 Su Machiavelli, si possono vedere Q. Skinner, Machiavelli (ed. or. 1981), il Mulino, Bologna 1999; J.P. McCormick, Machiavellian Democracy, Cambridge University Press, Cambridge 2011. Per una riflessione complessiva, che da Machiavelli procede in una disamina dei successi e dei lati oscuri della tradizione repubblicana, cfr. L. Baccelli, Critica del repubblicanesimo, Laterza, Roma-Bari 2003.

${ }^{20}$ Le opere politiche principali di Machiavelli si possono leggere in N. Machiavelli, Opere, a cura di R. Rinaldi, 2 voll., Utet, Torino 1999. 
L'importanza del conflitto che oppone diversi gruppi sociali - più che singoli individui desiderosi di accrescere il proprio potere personale - per l'evoluzione delle soluzioni istituzionali non può essere sottostimata: l'esito è un approccio di tipo realista alla politica, che abbandona il favore classico - da Aristotele a Tommaso d'Aquino - verso l'armonia e la concordia, nella convinzione della necessità di fare i conti con la realtà della natura umana. Il modo in cui questo tema viene declinato dal Fiorentino tradisce, tuttavia, un pregiudizio filopopolare. Per Machiavelli, infatti, non troppo diversamente da Marsilio da Padova, la moltitudine, non solo è mossa dall'unico desiderio di non essere dominata, ma è più saggia $\mathrm{e}$ costante del sovrano ${ }^{21}$. Ciò comporta un ribaltamento della logica dietro alla teorizzazione del governo misto: non si tratta tanto di limitare i rischi del governo popolare quanto di porre un freno alla tendenza da parte dei principi di abusare del loro potere ${ }^{22}$.

\section{Origini dello Stato}

L'esordio del Principe di Machiavelli testimonia la comparsa di un nuovo protagonista della politica, vale a dire lo Stato ${ }^{23}$. Lo Stato moderno - dalla locuzione latina status rei publicae (condizione della 'repubblica') - nasce in Europa dopo la dissoluzione del sistema feudale, basato su legami di tipo personale - vale a dire, il sovrano si accorda con i nobili del regno affinché questi esercitino parte delle sue prerogative, per esempio, in materia di esazione dei tributi, in cambio di appoggio militare - e dopo il tramonto del potere imperiale, dalla trasformazione delle grandi monarchie ereditarie - in primo luogo, Francia e Inghilterra.

Si tratta di una vicenda che si compie tra il XV e nel XVII secolo e che troviamo riflessa negli scritti di autori come Machiavelli, Jean Bodin (1529-1596) e Thomas Hobbes (1588-1679). L'elemento caratteristico di questa trasformazione è dato dall'accentramento in capo al sovrano e ai suoi incaricati delle funzioni che nel sistema feudale rimanevano decentrate: esazione dei tributi, produzione di diritto, amministrazione della giustizia, organizzazione militare. Emblematica è la vicenda

${ }^{21}$ Vedi N. Machiavelli, Discorsi sulla prima deca di Tito Livio, lib. I, 58, in Id., Opere, cit., pp. 705-714.

${ }^{22}$ Vedi L. Baccelli, Political Imagination, Conflict, and Democracy: $M a-$ chiavelli's Republican Realism, in D.C. Johnston, N. Urbinati, C. Vergara (eds.), Machiavelli on Liberty and Conflict, The University of Chicago Press, Chicago 2017, pp. 352-372.

${ }^{23}$ In generale sullo Stato, cfr.: N. Bobbio, Stato, governo, società. Frammenti di un dizionario politico, Einaudi, Torino 1995; N. Matteucci, Lo Stato moderno, il Mulino, Bologna 1993; P.P. Portinaro, Stato, il Mulino, Bologna 1999; M. Fioravanti (a cura di), Lo Stato moderno in Europa. Istituzioni e diritto, Laterza, Roma-Bari 2017. 
che si compie in Francia con Luigi XIII e Luigi XIV nel XVII secolo ${ }^{24}$. L'approdo finale è un assetto istituzionale che si caratterizza per la centralità del sovrano, unico detentore del monopolio della coazione legittima in un certo territorio: lo Stato assoluto. Questo esito si può dare per acquisito con la Pace di Westfalia, nel 1648, che segna la fine della Guerra dei Trent'Anni e consacra lo Stato come l'unico attore della politica internazionale.

Ovviamente, il processo tramite il quale si compie questo percorso non è istantaneo. Una fase intermedia si ha con il cosiddetto Stato di ceti. La caratteristica essenziale di questa tipologia di assetto politico è che il sovrano si contrappone non a un insieme omogeneo di sudditi ma a un insieme di ordini distinti, congiuntamente rappresentativi delle diverse componenti sociali presenti all'interno del regno e singolarmente portatori di specifiche prerogative ${ }^{25}$. I ceti trovano una loro forma di rappresentanza in assemblee consultive - per esempio, in Francia, l'assemblea degli Stati Generali: Nobiltà, Clero e Terzo Stato, che si conserverà fino alle soglie della rivoluzione - o giuridico/esecutive - come, sempre in Francia, i Parlamenti, tra cui il Parlamento di Parigi. In progresso di tempo, tuttavia, il rilievo politico di queste assemblee si andrà riducendo, mano a mano che si rafforza il potere del sovrano.

È importante notare che queste forme di rappresentanza si basano sul principio secondo il quale ciascun membro rappresenta gli interessi della categoria cui appartiene, operando come un delegato. Questo dato differenzia la logica rappresentativa dello Stato di ceti da quella dello Stato rappresentativo moderno, che, come vedremo, è basata sull'idea di una rappresentanza astratta dell'intero corpo politico - in questo caso si dice che il rappresentante opera come un fiduciario $^{26}$.

\section{I livellatori e la prima rivoluzione inglese}

Uno snodo fondamentale per la genesi di questo secondo concetto di rappresentanza si incontra in Inghilterra nel corso del 1600.

${ }^{24}$ Sul tema, cfr. A. De Benedictis, Politica, governo e istituzioni nell'Europa moderna, il Mulino, Bologna 2001; L. Mannori, Le istituzioni politiche dell'antico regime, in M. Meriggi, L. Tedoldi (a cura di), Storia delle istituzioni politiche. Dall'antico regime all'era globale, Carocci, Roma 2014, pp. 15-36.

${ }_{25}$ Vedi Mannori, Le istituzioni politiche dell'antico regime, cit., pp. $22 \mathrm{sgg}$.

${ }_{26}$ Sul concetto di rappresentanza, cfr. H.F. Pitkin, Il concetto di rappresentanza (ed. or. 1967), Rubbettino, Soveria Mannelli 2017; H. Hofmann, Rappresentanzarappresentazione. Parola e concetto dall'antichità all'Ottocento (ed. or. 1974), Giuffrè, Milano 2007; B. Accarino, Rappresentanza, il Mulino, Bologna 1999; G. Duso, La rappresentanza politica. Genesi e crisi del concetto, FrancoAngeli, Milano 2003; N. Urbinati, Representative Democracy: Principles and Genealogy, The University of Chicago Press, Chicago 2006. 
L'assetto inglese all'inizio del secolo era notevolmente diverso da quello delle monarchie continentali, in primo luogo Francia e Spagna, già incanalate verso lo Stato assoluto e verso il declino delle forme di rappresentanza cetuale. Le caratteristiche specifiche che contraddistinguevano il modello inglese erano date dal fatto che il potere del re era controbilanciato: $a$ ) da una parte, dal Parlamento, suddiviso in due camere - la Camera dei Lord, ereditaria, in cui sedevano i rappresentanti della nobiltà e dell'alto clero, e la Camera dei Comuni, elettiva, in cui sedevano i rappresentanti degli strati più elevati della nascente borghesia; b) dall'altra, da un corpo di giudici indipendenti, che interpretavano e al contempo sviluppavano il common law, il diritto comune, di formazione consuetudinaria, cui pure il re si riteneva fosse soggetto ${ }^{27}$.

Nella prima metà del XVII secolo i contrasti fra Giacomo I Stuart e, dal 1625, Carlo I Stuart e il Parlamento si accrebbero. Alla base di questa escalation si situa il disegno del sovrano di accentrare su di sé il potere, limitando le prerogative parlamentari. Il contesto, peraltro, vede una profonda convergenza tra rivendicazioni politiche e motivazioni religiose. Infatti, il disegno egemonizzante della Corona era stato perseguito anche attraverso la promulgazione nel 1534 dell'Atto di Supremazia, con il quale il sovrano inglese si costituiva come vertice della Chiesa anglicana, istituendo un nesso tra gerarchie ecclesiastiche e politiche. Questa mossa era profondamente invisa alle componenti più radicali del protestantesimo inglese - chiamate dispregiativamente Puritanes -, che invocavano il riconoscimento della piena autonomia di ciascuna comunità ecclesiale ${ }^{28}$.

L'esito fu la guerra civile nella quale la fazione dei Puritani, che rappresentava gli interessi della nascente borghesia in opposizione a quelli della nobiltà terriera, prevalse, sotto la guida di Oliver Cromwell. Carlo I fu giustiziato nel 1649 e fu instaurata la Repubblica. Questa non ebbe lunga vita, dal momento che si trasformò rapidamente in una sorta di dittatura di Cromwell, nominato Lord Protettore. Dopo la morte di questi, nel 1658, la monarchia degli Stuart venne restaurata. La breve fase repubblicana costituisce tuttavia un episodio determinante, dal momento che l'Inghilterra divenne il primo grande Stato repubblicano e rappresentativo - il Parlamento veniva eletto con suffragio censitario - dell'Europa moderna ${ }^{29}$.

${ }^{27}$ Sulla storia del costituzionalismo inglese è ancora utile C.H. MacIlwain, Costituzionalismo antico e moderno (ed. or. 1947), il Mulino, Bologna 1990.

${ }^{28}$ Cfr. M. Walzer, La rivoluzione dei santi. Il puritanesimo alle origini del radicalismo politico (ed. or. 1982), Claudiana, Torino 1996.

${ }^{29}$ Sulla storia inglese dell'epoca Stuart, cfr. Ch. Hill, The Century of Revolution, 1603-1714, Routledge, Abingdon 2001. 
Nei dibattiti che precedettero l'istituzione della Repubblica si scontrarono diverse voci, facenti capo ad altrettanti partiti formatisi in seno alla fazione puritana. Il movimento dei Livellatori - Levellers -, capeggiato da John Lilburne, Richard Overton e William Walvyn, si segnalò per un indirizzo spiccatamente democratico e borghese, che si sostanziava nella proposta del suffragio universale maschile. Il testo più famoso del movimento, An Agreement of the Free People of England, del 1649 ${ }^{30}$, è un manifesto in cui l'attitudine individualista si esprime nella rivendicazione della libertà di coscienza, dell'uguaglianza politica e del diritto di proprietà ${ }^{31}$, senza peraltro intaccare gli assetti economici sottostanti.

Ancora più radicale fu il movimento degli Zappatori - Diggers -, capeggiato da Gerrard Wistanley, che univa alle rivendicazioni democratiche l'idea secondo la quale la rivoluzione doveva mettere capo a una completa renovatio della società. Winstanley nella sua opera maggiore, The Law of Freedom ${ }^{32}$, criticava, da un lato, la forma di governo monarchica, che andava rimpiazzata dalla repubblica, dall'altro, il sottostante assetto economico. Sotto accusa era soprattutto la ripartizione diseguale della proprietà terriera - che dal secolo precedente era oggetto di appropriazione esclusiva tramite il sistema delle enclosures ${ }^{33}$, alla base dell'ascesa della nascente classe borghese - che nell'ottica degli zappatori doveva essere suddivisa ex novo secondo logiche egualitarie.

\section{Il modello contrattualista}

Gli anni della prima rivoluzione inglese sono anche gli anni nei quali Thomas Hobbes pubblica le sue opere politiche maggiori - il De Cive e il Leviatano ${ }^{34}$.

${ }^{30}$ Un'edizione recente dei testi dei Levellers è A. Sharp (ed.), The English Levellers, Cambridge University Press, Cambridge 2008. Per una traduzione italiana, cfr. V. Gabrieli (a cura di), Puritanesimo e libertà. Dibattiti e libelli, Einaudi, Torino 1956.

${ }^{31}$ David Wotton sostiene che si tratti della prima proposta di costituzione scritta basata sui diritti inalienabili. Vedi D. Wotton, Leveller Democracy and the Puritan Revolution, in J.H. Burns, M. Goldie (eds.), Cambridge History of Political Thought, 1450-170o, Cambridge University Press, Cambridge 1991, pp. 412-442: 412. Cfr. anche Ch. Hill, The World Turned Upside Down: Radical Ideas During the English Revolution, Penguin, London 1991.

${ }^{32}$ G. Winstanley, The Law of Freedom and other Writings (ed. or. 1652), Cambridge University Press, Cambridge 2008.

33 Vale a dire, le recinzioni con cui vecchi e nuovi proprietari sottraevano la terra all'uso comune, destinandola soprattutto al pascolo delle pecore, la cui lana alimentava il mercato manifatturiero.

34 Sul pensiero politico di Hobbes, si possono consultare: G. Kavka, Hobbesian Moral and Political Theory, Princeton University Press, Princeton 1986; J. Hampton, Hobbes and the Social Contract Tradition, Cambridge University Press, Cambridge 1986; N. Bobbio, Thomas Hobbes, Einaudi, Torino 1989; R. 
Le opere politiche di Hobbes segnano un avvenimento epocale nella storia del pensiero politico perché delineano chiaramente le coordinate di un nuovo modello di giustificazione del potere, alternativo a quello aristotelico. Nel modello aristotelico, come ho sottolineato nel capitolo precedente, il dato di partenza era la presenza di un cosmo ordinato da relazioni gerarchiche, nel quale gli individui si presentano segnati da marcate disuguaglianze e in cui le formazioni sociali - famiglia, villaggio, polis - si impongono come fenomeni naturali, la cui costituzione segue pulsioni endogene e il cui fine consiste nel perseguimento di un bene comune predefinito. Al contrario, per Hobbes il mondo si presenta, secondo i dettami della scienza del tempo, come un universo retto da leggi meccanicistiche, abitato da individui sostanzialmente uguali fra loro, ciascuno dei quali è proteso verso l'acquisizione delle risorse necessarie per soddisfare i propri desideri ${ }^{35}$.

Dal momento che nello stato di natura ciascuno ha diritto a tutto ciò che reputi necessario per la sua conservazione ${ }^{36}$, il risultato è una condizione perpetua di guerra di tutti contro tutti - homo homini lupus - dalla quale è possibile uscire soltanto attraverso un accordo fra tutti gli individui. Pertanto, vi è un prima, lo stato di natura, che consiste in un conflitto perpetuo; e un dopo, la società civile in cui la moltitudine diventa popolo. L'evento che separa le due fasi è un artificio, un patto che è frutto di una scelta razionale da parte degli individui, con il quale essi si accordano per cedere i propri diritti - eccetto il diritto all'autodifesa - a un terzo, il sovrano - che può essere un solo uomo oppure un'assemblea -, generato dal patto stesso, di cui non è parte e da cui non è quindi vincolato ${ }^{37}$.

Hobbes è spesso ritenuto il fondatore della tradizione contrattualista in filosofia politica. Questa affermazione, tuttavia, deve essere precisata. Infatti, da un lato la tradizione contrattualista è molto più risalente, vantando origini medievali - motivi proto-contrattualistici si possono rinvenire, per esempio nel Defensor pacis di Marsilio da Padova. Hobbes, però, si distacca dalle elaborazioni precedenti sotto due punti di vista. In primo luogo, perché impone allo strumento del contratto una decisa torsione individualistica: le parti contraenti non sono più entità collettive - membri di una certa comunità, per esempio, i nobili del regno,

Tuck, Hobbes (ed. or. 1989), il Mulino, Bologna 2001; A.P. Martinich, Hobbes, Routledge, Abingdon 2005.

35 Sull'antropologia di Hobbes e sul suo rilievo per la politica, cfr. D. D’Andrea, Prometeo e Ulisse. Natura umana e ordine politico in Thomas Hobbes, La Nuova Italia Scientifica, Roma 1997.

${ }^{36}$ Vedi Th. Hobbes, Leviatano (ed. or. 1651), a cura di R. Santi, Bompiani, Milano 2001, parte I, cap. 14, 4, pp. 213-215.

${ }^{37}$ Ivi, parte II, cap. 17,13 , pp. 281-283. 
gli abitanti di una città - ma individui uguali fra loro e non accumunati da precedenti legami sociali. Inoltre, e conseguentemente, l'espediente del contratto non serve a limitare il potere del sovrano, come in passato, ma a fondarlo ${ }^{38}$.

Il sovrano rappresenta - con una torsione rispetto al significato premoderno di rappresentanza, secondo il quale il rappresentante era il portavoce di interessi di parte - l'interesse universale dell'intero popolo e con ciò diventa il detentore dell'unica autorità, che non discende dall'alto come nel Medioevo ma viene costruita dal basso, dal pactum unionis degli individui ${ }^{39}$. Al tempo stesso, il conflitto viene estinto tramite la riconduzione della moltitudine, divenuta popolo, al principio di unità del sovrano. La costruzione del politico viene, così, completamente secolarizzata, rimuovendo gli assunti teologici che consentivano di fondare il potere. Di converso, la religione viene politicizzata, divenendo una componente del disegno complessivo che conduce al consolidamento delle istituzioni.

La strategia hobbesiana sovverte la soluzione 'democratica' al problema di trovare la migliore forma di governo messa in campo da Aristotele. Aristotele, come si ricorderà, sosteneva, in risposta alle critiche di Platone, che anche un vasto insieme di persone può disporre delle capacità necessarie per governare, a patto che i membri di questo insieme siano in qualche modo 'qualificati'. Pertanto, l'efficacia dell'azione di governo viene a dipendere, in ultima analisi, dalle differenze naturali tra gli individui e dalla selezione di coloro che dispongono delle 'giuste' capacità per essere cittadini ${ }^{40}$. Ora, nel momento in cui Hobbes tematizza la naturale uguaglianza degli uomini, questa soluzione non è più disponibile. Non si può fare affidamento sulla verità della natura per assicurare la bontà dell'azione dei governanti ma bisogna ricorrere a un'autorità creata dagli individui stessi. Ma questa mossa rende problematico ogni tentativo di rilevarne la contrarietà ai principî di giustizia. $\mathrm{E}$ infatti, Hobbes elimina il problema dell'eventuale ingiustizia del sovrano secondo la massima: «auctoritas, non veritas facit legem»" ${ }^{41}$ Nel quadro

${ }^{38}$ Sul tema, cfr. J. Sommerville, The Social Contract (Contract of Government), in G. Klosko (ed.), The Oxford Handbook of History of Political Philosophy, Oxford University Press, Oxford 2011, pp. 573-585.

39 Sulle novità della nozione hobbesiana di rappresentanza, rimane classico Pitkin, The Concept of Representation, cit. Per una discussione recente, cfr. D. Runciman, Hobbes's Theory of Representation: Anti-democratic or Proto-democratic?, in I. Shapiro et al., Political Representation, Cambridge University Press, Cambridge 2009, pp. 15-34.

${ }_{40} \mathrm{Nel}$ senso che, per Aristotele, un vasto insieme di schiavi non sarebbe idoneo a esercitare funzioni di governo, stante l'inferiorità per natura degli schiavi.

${ }^{41}$ La massima figura nell'edizione latina del Leviatano del 1688. Vedi Hobbes, Leviatano, cit., parte II, capp. 26, 21, p. 448. 
dell'impianto hobbesiano non è possibile garantire la bontà dell'azione del sovrano perché non sussistono standard di giustizia indipendenti dalla sua autorità. Il potere del sovrano è illimitato e le sue leggi non traggono forza dal conformarsi a una legge naturale anteriore ma dalla sua volontà, che è espressione della volontà dell'intero corpo politico. In questo modo, Hobbes legittima teoricamente lo Stato assoluto.

Da quanto detto, sembrerebbe che Hobbes non attribuisca alcuno spazio alla democrazia. Tuttavia, una simile conclusione sarebbe affrettata. Infatti, come numerosi commentatori hanno rilevato ${ }^{42}$, il pactum unionis, che istituisce il sovrano, è un accordo concluso tra individui uguali fra di loro, quindi sembra presupporre una logica di tipo orizzontale, in contrasto con il 'verticalismo' dei modelli medievali. Questa considerazione sembra rinforzare la tesi, che Hobbes stesso aveva espresso nei giovanili Elementi di legge naturale e politica, che la forma di governo democratica è anteriore alla monarchia e all'aristocrazia, perché il conferimento di potere a un singolo o a pochi presuppone un precedente accordo della maggioranza ${ }^{43}$.

Ciò non significa peraltro che la teoria hobbesiana sia democratica come pure è stato sostenuto. L'idea, piuttosto, è che il principio ugualitario che è alla base della forma di governo democratica gode di una priorità teorico-genetica, nella misura in cui per fondare il potere non si può non ricorrere a una procedura 'dal basso', secondo uno schema familiare ai teorici medievali dall'interpretazione della lex regia de imperio ${ }^{44}$. Più ancora, la rilevanza di Hobbes per la storia della democrazia diventa evidente se si adotta una prospettiva più larga: è la svolta materialista e individualista rappresentata dal contrattualismo hobbesiano che pone le condizioni perché le concezioni democratiche moderne possano svilupparsi.

\section{La Glorious Revolution e Locke}

Dopo la restaurazione della monarchia degli Stuart sorsero rapidamente nuove tensioni fra il sovrano e il Parlamento, impegnato nel ten-

${ }^{42}$ Da ultimo, vedi S. Petrucciani, Democrazia, Einaudi, Torino 2014, pp. 44-45.

43 Vedi Th. Hobbes, Elementi di legge naturale e politica (ed. or. 1640), a cura di A. Pacchi, La Nuova Italia, Firenze 1968, parte II, cap. 2, 1, pp. 177-178. Cfr. anche: Th. Hobbes, De cive. Elementi filosofici sul cittadino (ed. or. 1642), a cura di T. Magri, Editori Riuniti, Roma 1979, capp. 7, 1 sgg., pp. 145 sgg.; Id., Leviatano, cit., parte II, capp. 19, 1, pp. 303-305.

${ }_{44}$ Cfr. R. Tuck, Hobbes and Democracy, in A. Brett, J. Tully, H. Hamilton-Bleakley (eds.), Rethinking the Foundations of Modern Political Thought, Cambridge University Press, Cambridge 2006, pp. 171-190, e le critiche di K. Hoekstra, A Lion in the House: Hobbes and Democracy, in Rethinking the Foundations of Modern Political Thought, cit., pp. 219-235. Cfr. anche J. Martel, Subverting the Leviathan: Reading Thomas Hobbes as a Radical Democrat, Columbia University Press, New York 2007. 
tativo di difesa delle proprie prerogative politiche dalle mire accentratrici della monarchia. Tuttavia, questa volta non si giunse alla guerra civile: si ebbe invece una sollevazione meno cruenta ${ }^{45}$, che diverrà nota con il nome di Glorious Revolution: il re Giacomo II fuggì mentre il Parlamento, nel 1688, conferiva i poteri a Guglielmo d'Orange, che aveva sposato la figlia di Giacomo II, Mary Stuart. Al tempo stesso, il Parlamento fissava con il Bill of Rights i limiti del potere del sovrano ${ }^{46}$.

Questo documento, punto di approdo di una lunga tradizione costituzionalista che ha il suo più remoto antesignano nella Magna Charta Libertatum del 1215 - la 'dichiarazione' con la quale il re Giovanni Senzaterra sanciva i diritti dei propri feudatari -, oltre a confermare il diritto alla libertà personale, fissava alcuni principî che diventeranno cardini dello Stato liberale moderno: i membri del Parlamento sono scelti con libere elezioni, nel Parlamento deve regnare la massima libertà di parola e di discussione, il Re non può disapplicare le leggi approvate dal Parlamento, il Re non può imporre nuove tasse senza il consenso del Parlamento.

Dalla Glorious Revolution emergeva in sostanza uno Stato moderno nel quale la nascente classe borghese trovava un canale di rappresentanza politica nella Camera dei Comuni, i cui membri venivano eletti con metodo censitario - mentre la Camera dei Lord era composta da rappresentanti dall'aristocrazia e delle gerarchie ecclesiastiche.

Le coordinate fondamentali del sistema politico che segue alla Glorious Revolution si ritrovano puntualmente nel pensiero di John Locke (1632-1704) ${ }^{47}$. Dopo aver escluso, nel Primo trattato sul governo, una fondazione puramente teologica del potere politico, accettando così implicitamente il punto di vista razionalistico hobbesiano, Locke, nel Secondo trattato sul governo, pubblicato nel 1689 ma scritto alcuni anni prima, propone una nuova giustificazione contrattualista del potere. In primo luogo, Locke offre una caratterizzazione dello stato di natura molto differente da quella di Hobbes. Mentre per Hobbes lo stato prepolitico era una condizione conflittuale, Locke ritiene che lo stato di natura

${ }^{45}$ La Glorious Revolution non fu totalmente pacifica: molti cattolici vi persero la vita.

${ }^{46}$ Cfr. ancora: Hill, The Century of Revolution, 1603-1714, cit. Il testo del Bill of Rights è disponibile in traduzione italiana: The Bill of Rights, introduzione di E. Wicks, Liberilibri, Macerata 2010.

47 Sul pensiero politico di Locke, cfr.: J. Dunn, Il pensiero politico di Locke (ed. or. 1979), il Mulino, Bologna 1992; R. Ashcraft, Revolutionary Politics and Locke's Two Treatises of Government, Princeton University Press, Princeton 1986; A. Tuckness, Locke and the Legislative Point of View: Toleration, Contested Principles, and Law, Princeton University Press, Princeton 2002. 
presenti un ordine proprio, che deriva, in ultima analisi, dall'assetto dato da Dio con le sue leggi ${ }^{4}$.

In particolare, nello stato di natura - tratteggiato da Locke in modo più realistico di quanto non facesse Hobbes, anche per la maggiore familiarità con i racconti di viaggio che dipingevano la vita degli indiani del Nuovo Mondo ${ }^{49}$ - gli individui, che si trovano in una situazione di uguaglianza, intesa come assenza di gerarchie ${ }^{50}$, posseggono già alcuni diritti inalienabili, che il potere politico dovrà confermare e tutelare. Essi sono detentori del diritto alla vita, alla libertà personale e alla proprietà di quei beni che sono frutto del loro lavoro - la proprietà è infatti una proiezione sul mondo esterno - dato da Dio agli uomini per il loro sostentamento - della fondamentale auto-appartenenza che contraddistingue gli individui ${ }^{51}$. Tuttavia, tali diritti non sono, nello stato di natura, adeguatamente tutelati, per l'assenza di giudici terzi che possano sanzionarne le violazioni. Ragione per cui si rende necessario uscire dallo stato di natura ed entrare nella società civile ${ }^{52}$.

Vale la pena notare come l'enfasi lockiana sulla proprietà acquisita tramite il lavoro valga a legittimare le rivendicazioni della nascente borghesia, dal momento che le fortune dei proto-borghesi derivavano dalla recinzione e successivo sfruttamento di terre sottratte all'uso comune. Questo punto si lega a un fondamentale elemento dell'antropologia politica lockiana: l'uguaglianza spirituale, davanti a Dio, può coesistere con la disuguaglianza economica ${ }^{53}$. Locke si spinge sino a dire che l'accumulazione di beni diventa possibile nel momento in cui, grazie all'invenzione della moneta, si scongiura il rischio del loro deperimento, con ciò giustificando ante litteram l'accumulazione di capitale ${ }^{54}$.

${ }^{48}$ Locke aveva dedicato al tema delle leggi di natura un suo saggio giovanile, J. Locke, Saggi sulla legge di natura, Laterza, Roma-Bari 1973. Le più importanti opere politiche sono i due Trattati sul governo, J. Locke, Due trattati sul governo e altri scritti politici (e il Patriarca di Robert Filmer), a cura di L. Pareyson, Utet, Torino 1982.

49 Vedi Locke, Secondo trattato sul governo (ed. or. 1689), cit., cap. VIII, 102, pp. 301-302. Cfr. anche J. Tully, Rediscovering America: The Two Treatises and Aboriginal Rights, in Id., An Approach to Political Philosophy: Locke in Contexts, Cambridge University Press, Cambridge 1993, pp. 137-176.

${ }^{5}$ Vedi Locke, Secondo trattato sul governo, cit., cap. II, 4, p. 229.

${ }^{51}$ Ivi, cap. II, 6, pp. 231-232.

${ }^{52}$ Ivi, cap. IX, pp. 318-323.

53 Cfr.: J. Waldron, God, Locke, and Equality: Christian Foundations in Locke's Political Thought, Cambridge University Press, Cambridge 2002, pp. 116-117, 226227; J. Israel, Una rivoluzione della mente. L'illuminismo radicale e le origini intellettuali della democrazia moderna (ed. or. 2009), Einaudi, Torino 2011, p. 87.

${ }^{54}$ Ivi, cap. V, trad. it. cit., pp. 247-263. L'interpretazione proto-capitalistica del pensiero di Locke è stata sviluppata in particolare da Crawford Macpherson. Cfr. C.B. Macpherson, Libertà e proprietà alle origini della società borghese (ed. or. 1962), 
L'ingresso nella società civile si compie attraverso un patto con il quale gli individui si accordano per cedere i propri diritti al corpo politico, che diventa sovrano ${ }^{55}$. Tale sovranità si manifesta nel potere di fare le leggi. Questo risiede presso il popolo, che può delegarlo a propri rappresentanti eletti. Il corpo politico però non potrà violare i diritti naturali alla vita, alla libertà e alla proprietà, ma anzi si assume il compito di garantirli56 ${ }^{5}$. Il potere legislativo è distinto e sovraordinato al potere esecutivo, vale a dire il potere di applicare le leggi e punire i trasgressori e al potere federativo, cioè il potere di gestione della politica estera, entrambi esercitati da un fiduciario delegato a ciò dal legislativo ${ }^{57}$.

Da queste premesse derivano i caratteri specifici che lo Stato lockiano possiede rispetto a quello hobbesiano. In primo luogo, non si tratta di uno Stato assoluto, dato che il potere del sovrano è limitato dai diritti naturali; inoltre, il potere legislativo viene esercitato da un Parlamento eletto con metodo censitario che rappresenta il popolo sovrano e deve comunque rispettare i diritti naturali degli individui; il re, invece, che esercita il potere esecutivo e federativo, è investito dal legislativo tramite uno specifico patto.

Il modello lockiano, che avrà poi un'influenza determinante per gli sviluppi della democrazia moderna, mostra che si può evitare che la premessa dell'uguaglianza naturale degli uomini sfoci in una teorizzazione dell'assolutismo di stampo hobbesiano, a condizione che si concepiscano gli individui come già soggetti alle leggi di natura e quindi disciplinati/ disciplinabili ${ }^{5}$. Quindi, l'uguaglianza naturale è compatibile con la garanzia della giustizia dell'azione di governo a condizione che venga attivato un orizzonte normativo sovraordinato modellato sul profilo del cittadino proprietario.

\section{Spinoza}

Negli stessi anni in cui in Inghilterra si compiva un passo fondamentale verso la modernità politica, sintetizzato nel modello lockiano, nel contesto delle Province Unite d'Olanda, percorso da fortissime

Isedi, Milano 1973. Interpretazioni più moderne della dottrina lockiana della proprietà, tese a correggere gli anacronismi delle letture precedenti, si possono trovare in: J. Tully, A Discourse on Property. John Locke and His Adversaries, Cambridge University Press, Cambridge 1980; G. Sreenivasan, The Limits of Lockean Rights in Property, Oxford University Press, Oxford 1995.

55 Vedi Locke, Secondo trattato sul governo, cit., cap. VIII, pp. 297-317.

${ }^{56}$ Ivi, cap. XI, pp. 326-336.

57 Ivi, cap. XII-XIII, pp. 337-349.

${ }^{8} \mathrm{Su}$ questi temi, cfr. E. Santoro, Autonomia individuale, libertà e diritti. Una critica dell'antropologia liberale, Ets, Pisa 1999. 
tensioni sociali, economiche, politiche e religiose, matura la riflessione di Baruch Spinoza (1632-1677) ${ }^{59}$. Spinoza, tuttavia, benché erediti dagli autori contemporanei - segnatamente da Hobbes - concetti e problemi tipici del suo tempo, li elabora in una direzione divergente rispetto all'asse prevalente.

Questo assunto vale in particolare per la filosofia politica. Nel Trattato teologico-politico, infatti, Spinoza descrive una condizione naturale non molto dissimile da quella hobbesiana, nella quale il diritto di ciascun individuo si estende fin dove si estende la sua potentia ${ }^{60}$. Questo stato di cose rende necessario un patto costitutivo dello Stato, finalizzato alla costruzione della pace e dell'armonia ${ }^{61}$. Tuttavia, con questo patto i diritti individuali non sono trasferiti in capo a un sovrano monocratico, come nel caso hobbesiano, ma all'intera comunità, che pertanto darà vita a una democrazia $^{62}$. In questo modo, Spinoza rifiuta la mossa cruciale di Hobbes che consisteva nel derivare dal rilevamento dell'insostenibilità della situazione naturale, la necessità di una riconduzione del conflitto politico all'unità del sovrano. Analogamente, per quanto riguarda il ruolo politico della religione, mentre Hobbes, come abbiamo visto, ne sottolineava soprattutto la portata politica in vista del rafforzamento delle istituzioni, Spinoza critica la fede superstiziosa e difende la tolleranza e la libertà di coscienza, proponendo una reinterpretazione complessiva della religione in chiave emancipatrice e democratica.

Nel successivo Trattato politico, rimasto incompiuto, Spinoza abbandona del tutto la cornice contrattualista, per delineare una 'fisiologia' del politico che articola la disamina delle diverse forme di governo sulla base delle fondamentali coordinate delle sue concezioni metafisiche e antropologiche. Ne emerge una teoria realista e materialistica, che si richiama in più punti all'eredità machiavelliana ${ }^{63} \mathrm{e}$ che riconosce nella moltitudine (non nel popolo hobbesiano) il fondamentale soggetto politico detentore

${ }_{59} \mathrm{Su}$ Spinoza politico si possono vedere: A. Matheron, Individu et communauté chez Spinoza, Les Editions de Minuit, Paris 1969; D.J. Den Uyl, Power, State and Freedom: An interpretation of Spinoza's Political Philosophy, Van Gorcum \& Company, Assen 1983; E. Balibar, Spinoza e la politica (ed. or. 1990), Manifestolibri, Roma 1996. Per un'interpretazione recente del ruolo delle dottrine di Spinoza nella storia del pensiero democratico, cfr. J. Israel, Radical Enlightenment: Philosophy and the Making of Modernity 1650-1750, Oxford University Press, Oxford 2002; Id., Una rivoluzione della mente, cit.

${ }^{60}$ Vedi B. Spinoza, Trattato teologico-politico (ed. or. 1670), in Id., Tutte le opere, a cura di A. Sangiacomo, Bompiani, Milano 2010, cap. 16, 2-4, pp. 1003-1007. Vedi anche Id., Trattato politico, (ed. or. 1677), cap. 2, 4, in Id., Tutte le opere, cit. p. 1637.

${ }^{61}$ Vedi B. Spinoza, Trattato teologico-politico, cit., cap. 16, 5, pp. 1007-1009.

${ }^{62}$ Ivi, cap. 16, 8-11, pp. 1013-1017.

${ }^{63}$ Sul nesso Machiavelli-Spinoza, cfr. F. Del Lucchese, Tumulti e indignatio. Conflitto, diritto e moltitudine in Machiavelli e Spinoza, Ghibli, Milano 2004. 
del potere. La moltitudine, infatti, «condotta come da una sola mente» ${ }^{64}$, determina il diritto dei poteri sovrani dello Stato.

Questo punto di vista conduce a un superamento della tradizionale tricotomia delle forme di governo. Spinoza sottolinea il momento della democratizzazione complessiva della società: monarchia e aristocrazia sono viste convergere verso un assetto nel quale l'imperium è in mano alla multitudo, senza peraltro cristallizzarsi in una forma definitiva. La democrazia, pertanto, lungi dall'essere una forma di governo fra le altre, «esprime la logica profonda della condizione 'civile' dell'umanità» ${ }^{65}$. Con ciò siamo alle soglie di una concezione 'radicale' ${ }^{66}$ della democrazia che, per la prima volta, si distacca consapevolmente dalle teorie medievali del governo misto.

\section{Montesquieu}

All'inizio del XVIII secolo il sistema inglese diventa il modello di riferimento in ambito europeo. La fortuna di questo modello è particolarmente evidente ne Lo spirito delle leggi di Charles-Louis de Secondat, barone di Montesquieu (1689-1755) ${ }^{67}$. L'attenzione che Montesquieu pone verso le istituzioni politiche inglesi è tanto più significativa in quanto esse vengono contrapposte implicitamente alla monarchia assoluta di Luigi XIV. Il difetto capitale del centralismo assolutista è quello di produrre un assetto instabile, privando di rappresentanza politica componenti significative della società. A questo difetto il modello inglese pone rimedio attraverso un'articolazione istituzionale bilanciata.

Lo Spirito delle leggi si presenta come una vasta indagine 'sociologica' sulle tre principali forme di governo: monarchia, repubblica e dispotismo. Montesquieu prende in esame comparativamente assetti politici distanti nel tempo e nello spazio. In questo modo, dovrebbe emergere il nesso esistente - invariabile, al di sotto del mutare degli elementi contingenti - tra ordinamento giuridico e politico e fattori di natura economica, religiosa, climatica e di costume.

Mentre la monarchia è la forma di governo nella quale il principe governa in accordo con le leggi e il dispotismo - storicamente associato al mondo orientale ma nel quale si legge, in filigrana, la critica dell'assolutismo contemporaneo - è la forma di governo nella quale il detentore del potere governa arbitrariamente attraverso la paura, la repubblica è

${ }^{64}$ Vedi Spinoza, Trattato politico (ed. or. 1677), cit., cap. 3, 2, p. 1651.

${ }_{65}$ P. Costa, Civitas. Storia della cittadinanza in Europa, vol. 1, Dalla civiltà comunale al Settecento, Laterza, Roma-Bari 1999, p. 231.

${ }^{66}$ Il riferimento è a Israel, Una rivoluzione della mente, cit.

${ }^{67} \mathrm{Su}$ Montesquieu, si può consultare J. Shklar, Montesquieu (ed. or. 1989), il Mulino, Bologna 1990. 
la forma di governo nella quale il potere sovrano è detenuto dal popolo. Montesquieu distingue inoltre tra due forme di repubblica, la repubblica aristocratica e la repubblica democratica, che si regge sulla virtù civica del popolo e sull'amore dell'uguaglianza.

Tuttavia, per Montesquieu la forma di governo repubblicana è legata al passato e a comunità politiche di ridotta estensione. Il suo limite principale consiste nel fatto di non garantire la libertà politica dei cittadini. La libertà politica, infatti, non consiste nel fare ciò che si vuole ma nel fare ciò che le leggi permettono, vale a dire, ciò che si deve volere ${ }^{68}$. La forma di governo che garantisce la maggiore libertà in questo senso, è la monarchia limitata, che, sulla base dell'esperienza inglese, viene definita attraverso la tesi della separazione dei poteri, ovvero la tesi secondo la quale i tre poteri fondamentali dello Stato - potere legislativo, potere esecutivo e potere giudiziario - devono fare capo a organi diversi e autonomi, capaci di limitarsi vicendevolmente. Questo assetto di potere è il solo che garantisca la libertà degli individui perché previene il rischio che il titolare di uno dei poteri possa abusarne.

In questo modo, il potere legislativo, il cui detentore è il popolo verrà in concreto esercitato dai suoi rappresentanti, eletti da tutti i cittadini che sono proprietari, senza vincolo di mandato ${ }^{69}$, all'interno di un Parlamento bicamerale. Il potere esecutivo verrà esercitato dal re, sotto il controllo del Parlamento. Infine, il potere giudiziario sarà appannaggio di giudici indipendenti, che si limiteranno ad applicare le leggi approvate dal Parlamento. Così, aggirata «l'anomalia» spinoziana ${ }^{70}$, il modello liberale di derivazione lockiana si appresta a diventare il sigillo della modernità politica, ripristinando la continuità con la dottrina del governo misto, nel segno di una concezione della libertà come adeguamento a un orizzonte normativo preesistente.

\section{Rousseau}

Benché le opere politiche maggiori di Jean-Jacques Rousseau (17121778) appaiano a pochi anni di distanza dallo Spirito delle leggi una grande distanza teorica le separa dall'opera di Montesquieu ${ }^{71}$. L'intento

${ }^{68}$ Vedi Montesquieu, Lo spirito delle leggi (ed. or. 1748), in Id., Tutte le opere [1721-1754], a cura di D. Felice, Bompiani, Milano 2014, lib. XI, cap. 3, p. 1217.

${ }^{69}$ Vale a dire senza essere vincolati dalle istruzioni specifiche di coloro che li hanno eletti, come avveniva in relazione alla rappresentanza di interessi di ascendenza medievale.

$7^{70}$ L'implicito riferimento è qui alla famosa interpretazione di Antonio Negri. Cfr. A. Negri, Spinoza. L'anomalia selvaggia. Spinoza sovversivo. Democrazia ed eternità in Spinoza, Deriveapprodi, Roma 1998.

${ }^{71} \mathrm{Su}$ Rousseau, cfr.: A. Ferrara, Modernità e autenticità. Saggio sul pensiero sociale ed etico di J.J. Rousseau, Armando, Roma 1989; R. Wokler, Rousseau (ed. 
di Rousseau è infatti opposto: mentre Montesquieu si proponeva di riflettere su quali assetti istituzionali fossero più idonei alla garanzia della libertà, Rousseau elabora attraverso gli strumenti concettuali forniti dal contrattualismo una moderna teoria politica, basata sull'idea della sovranità del popolo.

Il punto di partenza del discorso di Rousseau è una caratterizzazione dello stato di natura opposta a quella di Hobbes: per Rousseau, infatti, lo stato di natura è una condizione felice nella quale ciascun individuo possiede i mezzi per soddisfare i propri bisogni, sotto la guida infallibile delle leggi della natura ${ }^{72}$. Questa caratterizzazione, d'altra parte, non ha i connotati di realismo proto-antropologico che ha in Locke, né l'astrattezza assiomatica che possiede in Hobbes, assomigliando piuttosto al vagheggiamento di una perduta età dell'oro.

Tale condizione però si ribalta, in conseguenza del progresso che conduce alla scoperta dell'agricoltura e all'istituzione della proprietà, in uno stato sommamente infelice nel quale esplode la conflittualità fra gli individui ${ }^{73}$. L'infelicità che è propria della vita associata deve essere cancellata dalla politica. Si noti che, mentre in Hobbes lo stato di natura si contrapponeva direttamente alla società civile istituita dalla politica, nel discorso di Rousseau viene aggiunto uno stadio intermedio fra lo stato di natura e la società politica: la società civile come società civilizzata. La società civile rappresenta però un sovvertimento della felicità naturale $\mathrm{e}$ produce l'amplificazione delle differenze naturali fra gli individui. Diventa necessario, per ritornare alla felicità originaria, annullare le differenze che si sono prodotte.

Lo strumento idoneo per conseguire questo risultato può essere soltanto un patto tra tutti gli individui. Ma a questo punto si presenta una difficoltà: se la vita associata ha amplificato a dismisura le differenze naturali sussistenti fra gli individui, un patto del genere non potrebbe che rivelarsi iniquo, segnato dal predominio del più forte sul più debole. Diventa necessario quindi ristabilire l'uguaglianza naturale. Questa necessità non si poneva per Hobbes, per il quale gli individui, prima del patto, sono sostanzialmente uguali, né per Locke, per il quale l'uguaglianza che conta è quella di fronte alla legge, che permette l'esistenza di disuguaglianze economiche rilevanti.

or. 1995), il Mulino, Bologna 2001; N.J.H. Dent, Rousseau, Routledge, London 2005; J. Cohen, Rousseau: A Free Community of Equals, Oxford University Press, Oxford 2010. Gli scritti politici di Rousseau si possono leggere in J.J. Rousseau, Scritti politici, 3 voll., a cura di M. Garin, Laterza, Roma-Bari 1971.

${ }^{72}$ Vedi J.J. Rousseau, Discorso sull'origine e i fondamenti dell'ineguaglianza tra gli uomini (ed. or. 1755), parte I, in Id., Scritti politici, cit., vol. I, pp. 142-172.

${ }_{73}$ Ivi, parte II, pp. 173-205. 
Per ottenere questo esito Rousseau ritiene che gli individui debbano procedere a una completa alienazione dei propri diritti all'atto del loro ingresso nella società politica. In pratica, gli individui, per istituire la comunità politica, devono rinunciare a tutti i loro diritti naturali - a differenza di quanto avviene nel contrattualismo lockiano - a favore del corpo politico ${ }^{74}$. Peraltro, essi verranno loro restituiti sotto forma di diritti civili, secondo uno schema non troppo dissimile da quello spinoziano. La comunità politica risultante è una repubblica, nella quale la sovranità è attribuita al popolo, unico detentore del potere di approvare le leggi. La legge è espressione della volontà generale, che è la volontà della comunità ed è naturalmente diretta al bene comune. Essa non coincide con la somma delle volontà particolari, né con la volontà di tutti, ma le trascende, in quanto la sua aggregazione è stata resa possibile dalla rimozione delle differenze soggettive ${ }^{75}$. Questo passaggio è cruciale, in quanto stabilisce una differenza di livelli fra ciò che gli uomini effettivamente vogliono e ciò che devono volere. È questo scarto che assicura la razionalità delle decisioni che il corpo politico andrà ad assumere.

Dal punto di vista istituzionale, Rousseau sostiene che la sovranità non può essere rappresentata, in quanto non si può volere per altri ${ }^{76}$. Ciò non significa tuttavia che l'unica forma di governo legittima sia quella democratico diretta - tipica degli Stati di estensione territoriale ridotta perché il governo può essere esercitato anche da un'aristocrazia o persino da un monarca, a patto che questi soggetti si limitino a far eseguire le leggi approvate dal popolo ${ }^{77}$. Sta di fatto, però, che l'adozione del sistema rappresentativo mette a repentaglio la sovranità popolare. Per questo motivo, la delega può essere ammessa al più come mero espediente tecnico, volto a fronteggiare l'impossibilità del governo diretto da parte del popolo entro Stati territorialmente estesi, ma sempre con l'introduzione di meccanismi diretti a prevedere forme di controllo sull'attività del delegato ${ }^{78}$.

In definitiva, la riflessione politica di Rousseau, alla vigilia della rivoluzione francese, ribadisce, in altre forme, l'assunto caratteristico della teoria democratica, assunto che abbiamo trovato canonizzato già nella Politica di Aristotele: per sottrarsi al rischio di una degenerazione nella tirannide della maggioranza e quindi per mettere in condizione la politica

${ }^{74}$ Vedi J.J. Rousseau, Contratto sociale (ed. or. 1762), lib. I, cap. 6, in Id., Scritti politici, cit., vol. II, pp. 92-94.

75 Ivi, lib. II, cap. 3, pp. 104-105.

${ }^{76}$ Ivi, lib. III, cap. 15, pp. 162-165. La volontà del popolo può essere tuttavia espressa da commissari.

77 La disamina delle diverse forme di governo è oggetto del Libro III del Contratto sociale.

${ }^{78}$ Vedi J.J. Rousseau, Considerazioni sul governo della Polonia (ed. or. 1782 ), cap. 7, in Id., Scritti politici, cit., vol. III, pp. 200-216. 
di perseguire il bene comune è necessario introdurre qualche meccanismo idoneo a condizionare l'output politico. Tale meccanismo può essere relativo alla selezione dei detentori del potere, come nello stesso Aristotele e nei teorici del governo misto, oppure, quando l'ipotesi di uguaglianza antropologica renda tale selezione impossibile, alla previsione di un orizzonte normativo cui le decisioni politiche devono conformarsi. 



\section{Capitolo terzo \\ L'Ottocento}

\section{La rivoluzione americana}

Alla fine del Settecento si verificano due eventi che modificheranno profondamente la storia delle istituzioni politiche. Si tratta della rivoluzione americana e della rivoluzione francese.

Come è noto, nella seconda metà del secolo, esplodono le tensioni che contrapponevano le colonie americane alla madrepatria britannica. Queste tensioni, soprattutto di carattere economico, sfociarono in un vasto conflitto, cui presero parte anche la Francia, la Spagna e le Province Unite di Olanda: la guerra di indipendenza americana. Al termine di questo conflitto, la Gran Bretagna dovette riconoscere la nascita di un nuovo soggetto politico: gli Stati Uniti d'America. I documenti che segnano le tappe di questo processo sono la Dichiarazione di indipendenza (1776), la Costituzione federale (1787) - che fa seguito a numerose Costituzioni dei singoli Stati, prima fra tutte la Costituzione della Virginia - e il Bill of Rights (1791), che ne rappresenta i primi dieci Emendamenti ${ }^{1}$.

Gli elementi salienti della vicenda rivoluzionaria americana consistono, da un lato, nell'ascendenza giusnaturalistica: la premessa che legittima la Dichiarazione di indipendenza è il darsi di leggi naturali autoevidenti che rinviano alla volontà di Dio. Questa ascendenza si precisa secondo

${ }^{1}$ Sulla rivoluzione americana, cfr. N. Matteucci, La rivoluzione americana: una rivoluzione costituzionale, il Mulino, Bologna 1987; A. Taylor, Rivoluzioni americane. Una storia continentale, 1750-1804 (ed. or. 2017), Einaudi, Torino 2017. Il testo della Dichiarazione di indipendenza è leggibile in La Dichiarazione d'indipendenza degli Stati Uniti d'America, a cura di T. Bonazzi, Marsilio, Venezia 2001. Per la Costituzione federale e il Bill of Rights, vedi La Costituzione degli Stati Uniti. Storia, testo inglese, nuova traduzione, commento e note, a cura di F. Tonello, Mondadori, Milano 2010. 
coordinate lockiane: l'apparato istituzionale - che nella tripartizione di Presidente, Congresso, Corte Suprema riproduce il modello inglese di separazione dei poteri, Re, Parlamento, Giudici - è preordinato alla tutela dei diritti inviolabili degli individui - Vita, Libertà, Ricerca della felicità, che richiamano la triade lockiana: Vita, Libertà, Proprietà ${ }^{2}$

Al tempo stesso, il diritto di autogoverno da parte del popolo, enunciato nella Dichiarazione di Indipendenza, introduce un importante elemento riconducibile alla tradizione repubblicana (vedi cap. 2, par. 4) che si rende apprezzabile anche nel valore attribuito al pluralismo interno. Questi due assi si compongono nell'architettura repubblicana e federale del nuovo Stato. Il modello federale, imperniato sul bilanciamento tra i governi dei singoli Stati e il governo centrale raddoppia la separazione dei poteri che si realizza attraverso il balance of power di Presidente, Congresso, Corte Suprema ${ }^{3}$.

Il nuovo Stato si incentra, come detto, sul protagonismo del popolo - dove il riferimento al popolo acquista risonanze bibliche memori della vicenda dei Puritani inglesi che erano fuggiti in Nord America nel XVII secolo. Il legame tra popolo e potere legislativo viene però mediato dall'autorità della Costituzione: dalla prima metà dell'Ottocento si riconoscerà che la Corte Suprema può dichiarare l'invalidità delle leggi approvate dal Congresso - e dunque volute dal popolo - quando esse siano in contrasto con il dettato costituzionale.

Per altro verso, il nuovo Stato è una repubblica e non una democrazia. Questa differenza lessicale si collega al tradizionale pregiudizio secondo il quale la democrazia si sostanzia solo in una forma di governo diretto da parte del popolo, come tale impraticabile in Stati di grandi dimensioni. Al contrario, gli Stati Uniti sono una repubblica, possiedono una forma di governo nella quale la sovranità popolare si esprime in forme rappresentative, in contrasto con la concezione di Rousseau secondo la quale la sovranità popolare non può essere rappresentata. Nel complesso, l'assetto del nuovo Stato sembra conformarsi, per il tramite lockiano, all'archetipo tradizionale del governo misto.

Questo punto si lega al dibattito sul tema della rappresentanza. In alcuni articoli pubblicati nel 1787 e nel 1788 nel corso del dibattito sulla forma istituzionale del nuovo soggetto politico - e successivamente raccolti nel volume Il Federalista insieme ai contributi di Alexander Hamilton e John $\mathrm{Jay}^{4}$ - James Madison (1751-1836), uno degli ideologi dell'indipendenza e

${ }^{2}$ Sul tema, cfr. B. Casalini, Nei limiti del compasso: Locke e le origini della cultura politica e costituzionale americana, Mimesis, Milano-Udine 2002.

3 Sul versante repubblicano della rivoluzione, cfr. B. Bailyn, The Ideological Origins of the American Revolution, Harvard University Press, Cambridge, Mass. 1992.

4 Vedi A. Hamilton, J. Jay, J. Madison, The Federalist Papers (ed. or. 1788), trad. it. Il Federalista, a cura di G. Sacerdoti Mariani, Giappichelli, Torino 1997. 
futuro Presidente, critica aspramente la democrazia diretta e sostiene che la sovranità popolare può esprimersi soltanto in forme rappresentative. Secondo Madison - che riprendeva molto da vicino alcuni luoghi classici del dibattito antidemocratico, da Platone in poi - il rischio maggiore cui le democrazie tradizionali andavano soggette era quello della contrapposizione tra fazioni, che si traduceva in conflittualità prolungata $\mathrm{e}$, in ultima analisi, nella tirannide della maggioranza. A questi inconvenienti è necessario porre rimedio attraverso opportuni accorgimenti istituzionali, volti a rendere compatibili governo popolare e salvaguardia dei diritti. Il principale tra questi accorgimenti è il meccanismo elettivo unito all'istituto della rappresentanza ${ }^{5}$.

Madison si opponeva a una forma di rappresentanza nella quale il rappresentante si fa portavoce degli interessi particolari della categoria di soggetti che lo ha eletto. Madison, in polemica con Thomas Jefferson (1743-1826) - l'autore della Dichiarazione di indipendenza e terzo Presidente degli Stati Uniti -, ha in mente piuttosto una concezione aristocratica della rappresentanza, nella quale il rappresentante è esponente dell'élite illuminata della nazione e opera come filtro rispetto alle istanze provenienti dalla base, permettendo l'unificazione del corpo politico e la preservazione del pluralismo e prevenendo la radicalizzazione del conflitto. Questa idea, peraltro, si raccorda, nel dibattito dei Federalisti, alla duplice intuizione secondo la quale l'effetto selettivo è in generale prodotto dal funzionamento stesso del sistema elettorale, specialmente all'interno di collegi di grandi dimensioni, ed è associato alla prosperità economica ${ }^{6}$.

In questo modo, pertanto, viene consacrato il principio aristotelico, ereditato dai teorici del governo misto, in base al quale il popolo può esprimersi politicamente in forme non distruttive soltanto quando sia accompagnato da meccanismi di correzione e irreggimentazione della sua volontà. Questo effetto selettivo si produce in ragione della scelta dei rappresentanti, più che per effetto delle prescrizioni di un orizzonte normativo già dato, esplicitando così l'assunto sotteso al sistema lockiano, nel quale l'orizzonte della Legge naturale si modella sul profilo del cittadino borghese.

${ }^{5}$ Su questi temi, cfr. Manin, Principî del governo rappresentativo, cit., pp. 114-146. È opportuno ricordare che Madison accoglieva la tradizione precedente che associava il sorteggio dei magistrati alla democrazia e caratterizzava il sistema elettivo come non-democratico, per cui, anche da questo punto di vista, la selezione dei rappresentanti tramite elezioni vale a distinguere la repubblica dalla democrazia.

${ }^{6}$ Quest'ultimo punto è esplicito nel saggio 35 dei Federalist Papers, scritto da Hamilton. Cass Sunstein interpreta queste considerazioni come un'anticipazione dei principî-cardine della democrazia deliberativa. Vedi C.R. Sunstein, \#Republic. La democrazia nell'epoca dei social media, il Mulino, Bologna 2017, pp. 64-68. 


\section{La rivoluzione francese}

L'altro grande evento che segna la fine del XVIII secolo è la rivoluzione francese. Il dato di partenza, peraltro, è molto differente. L'assetto politico degli Stati Uniti discende da quello britannico, mentre il tessuto sociale non conosce l'articolazione in ceti tipica degli Stati europei. Viceversa, la società francese era segnata da profonde spaccature e disuguaglianze, nel contesto di uno Stato assoluto. Nel caso della rivoluzione francese, ma non di quella americana, si tratta quindi di introdurre una cesura profonda rispetto a un sistema preesistente. Questa necessità comporta una discontinuità istituzionale, non solo politica, ben più marcata ${ }^{7}$.

D'altra parte, proprio l'esperienza dello Stato assoluto aveva contribuito, in un certo senso, a preparare il terreno alla rivoluzione indebolendo i legami comunitari presenti all'interno del tessuto sociale francese. Infatti, il secolare sforzo della monarchia in direzione di un accentramento dei poteri aveva alimentato la cultura individualista che avrebbe poi fatto da sfondo alla rivoluzione. La rivoluzione completa questo secolare processo di trasformazione: dopo che l'esplosione rivoluzionaria aveva spazzato via le strutture dello Stato assoluto, l'Assemblea costituente intraprende una vasta riforma della società, eliminando ogni vestigia di articolazione cetuale, insieme ai privilegi della nobiltà e del clero. In questo modo, la fine dello Stato assoluto, ancora legato a una concezione organica della politica, in cui la gerarchia dei poteri corrisponde a un assetto naturale, lascia il passo all'edificazione di un moderno Stato rappresentativo, in cui il potere viene legittimato dal basso.

Il background individualista si ritrovava già perfettamente espresso nella Dichiarazione dei diritti dell'uomo e del cittadino del 1789, il grande documento che sintetizza la prima fase della vicenda rivoluzionaria ${ }^{8}$. In particolare, la Dichiarazione del 1789 afferma l'uguaglianza naturale degli uomini (art. 1), l'esistenza di diritti innati - tra i quali vengono menzionati il diritto alla vita, alla libertà, alla proprietà e alla resistenza contro l'oppressione - che l'associazione politica deve tutelare (art. 2),

7 Sulla rivoluzione francese, cfr. la nuova ricostruzione di Jonathan Israel, J. Israel, La rivoluzione francese. Una storia intellettuale dai Diritti delluomo a Robespierre (ed. or. 2015), Einaudi, Torino 2016, che applica le idee sviluppate nella trilogia precedente: Israel, Radical Enlightenment, cit.; Id., Enlightenment Contested, Enlightenment Contested: Philosophy, Modernity, and the Emancipation of Man 1670-1752, Oxford University Press, Oxford 2008; Id., Democratic Enlightenment: Philosophy, Revolution, and Human Rights 1750-179o, Oxford University Press, Oxford 2013.

${ }^{8}$ Dichiarazione dei Diritti dell'Uomo e del Cittadino (ed. or. 1789), introduzione di T.E. Frosini, Liberilibri, Macerata 2008. Sulla Dichiarazione, cfr. S. Rials, La déclaration des droits de l'homme et du citoyen, Hachette, Paris 1988. 
che la sovranità risiede nella Nazione (art. 3), che la legge è espressione - in accordo con Rousseau - della volontà generale, alla formazione della quale tutti i cittadini possono contribuire, in maniera diretta o tramite i propri rappresentanti (art. 6), che nessuno può essere accusato o detenuto se non nei casi e nelle forme previsti dalla legge (art. 7), che vige la presunzione di innocenza (art. 9), la libertà di religione, di parola, di stampa e la separazione dei poteri (art. 16).

Salta agli occhi il protagonismo del nuovo soggetto consacrato dalla Dichiarazione, vale a dire, la Nazione, presentato come detentore della sovranità e depositario di quella volontà generale che rousseauianamente trova espressione nella legge. L'identificazione della volontà generale con la volontà della Nazione, come espressa dai suoi rappresentanti, apre però il problema delle modalità nelle quali si concretizza la rappresentanza stessa. Da questo punto di vista, si presenta una situazione che possiede diversi punti di contatto con quella statunitense.

Da una parte, infatti, Emmanuel-Joseph Sieyès (1748-1836), nei dibattiti che avevano preceduto l'approvazione della Costituzione del 1791 - in cui il re conservava il potere esecutivo mentre il potere legislativo era affidato a un'assemblea eletta secondo criteri censitari -, aveva proposto un'idea della rappresentanza nella quale il rappresentante non è legato dalle istruzioni ricevute dalla base che lo ha eletto. Ogni rappresentante, al di là delle appartenenze personali, del proprio status e delle proprie ricchezze, rappresenta la volontà dell'intera Nazione, per questo motivo non può essere vincolato dal perseguimento di interessi particolari. Ciò non significa che l'elezione dei rappresentanti debba prescindere da requisiti censitari, ché anzi, implicita nel funzionamento della delega politica è l'idea che la loro investitura si risolva in una sorta di 'divisione del lavoro' tra $\mathrm{i}$ «cittadini attivi» - in sostanza, i francesi maschi proprietari - e i «cittadini passivi»9.

In questo modo, diventa possibile adattare il sistema rappresentativo innestandolo sul lessico teorico rousseauiano. Infatti, tramite la personificazione della Nazione si concretizza la distinzione tra volontà generale e volontà di tutti. Di conseguenza, la volontà generale, invariabilmente diretta al perseguimento del bene comune, viene a identificarsi con la volontà desumibile dall'operato dei rappresentanti della Nazione e quindi, in ultima analisi, con quella dei cittadini qualificati dai requisiti censitari.

9 Su Sieyès, cfr.: S. Mannoni, Une et indivisible. Storia dell'accentramento amministrativo in Francia, vol. I, La formazione del sistema (ed. or. 1661-1815), Giuffrè, Milano 1994, pp. 261-331; M. Goldoni, La dottrina costituzionale di Sieyès, Firenze University Press, Firenze 2009. Gli scritti di Sieyès si possono leggere in E.-J. Sieyès, Opere e testimonianze politiche, a cura di G. Troisi Spagnolo, Giuffrè, Milano 1993. 
Dall'altra, Maximilien de Robespierre (1758-1794), nel contesto del superamento repubblicano e democratico della Costituzione del $1791^{10}$, si schiera a favore di una concezione opposta a quella di Sieyès e molto più vicina a quella di Rousseau, nella quale i rappresentanti operano come commissari incaricati di esprimere la volontà soggiacente del popolo sovrano, privi quindi di un'autonoma facoltà di scelta e sottoposti al controllo popolare. Il presupposto di questa concezione è che, se i rappresentanti fossero chiamati a esprimere in maniera autonoma la volontà di tutta la Nazione, dovremmo ipotizzare l'esistenza di una volontà astratta cui l'azione concreta dei rappresentanti stessi si dovrebbe attenere, in contrasto con l'idea che la sovranità risiede nel popolo. Per tale motivo, Robespierre ritiene che la volontà del popolo, che si manifesta, sempre seguendo Rousseau, nell'Assemblea legislativa, non si lasci ingabbiare dentro le forme del potere costituito ma conservi la potenzialità per sovvertire ciascun ordine dato. Di conseguenza, ogni tentativo di distinguere tra categorie di cittadini diverse, con lo scopo di enucleare quale concezione del bene comune debba in concreto essere perseguita, va respinta, con ciò annullando la portata aristocratica della teoria sieyésiana della rappresentanza.

La parabola del Terrore rivoluzionario sembrerà dimostrare l'inadeguatezza della soluzione giacobina del problema della rappresentanza. Con la chiusura della parentesi rivoluzionaria e l'inizio della Restaurazione, la soluzione di Sieyès diventerà l'unica risposta accettabile all'interrogativo su come la Nazione debba essere rappresentata. Una soluzione che passa attraverso la ripresa del suffragio censitario e apre le porte all'affermazione politica della borghesia.

\section{Dopo la rivoluzione}

Al termine dell'avventura napoleonica le sorti d'Europa sono decise dalle potenze vincitrici nel Congresso di Vienna. Si apre la fase della Restaurazione $^{11}$. In generale, però le monarchie post-rivoluzionarie si

${ }^{10}$ Come è noto, dopo l'esecuzione di Luigi XVI e la proclamazione della repubblica nel 1792, la Convenzione, l'organo che ha sostituito l'Assemblea nazionale, approva una nuova Costituzione repubblicana e democratica. Questa Costituzione, però, che prevedeva il suffragio universale maschile e un cospicuo programma sociale - diritti all'istruzione e all'assistenza - non entrerà mai in vigore, giacché dal luglio del 1793 il governo rivoluzionario si trasforma in una sorta di dittatura della fazione giacobina attraverso il Comitato di Salute Pubblica, il cui leader diventerà in breve Robespierre. Un'antologia degli scritti di Robespierre è M. Robespierre, La Rivoluzione giacobina, a cura di U. Cerroni, Editori Riuniti, Roma 1967. Sulle concezioni della cittadinanza durante il periodo rivoluzionario, cfr. P. Costa, Civitas. Storia della cittadinanza in Europa, vol. 2, L'età delle rivoluzioni, Laterza, Roma-Bari 2000, cap. I.

${ }^{11}$ Sulla portata filosofico-politica della Restaurazione, cfr. S. Chignola, Il tempo rovesciato. La Restaurazione e il governo della democrazia, il Mulino, Bologna 
caratterizzano per il fatto di essere monarchie costituzionali, in cui il potere legislativo è esercitato, in tutto o in parte, da assemblee elette con metodo censitario. Così avviene in Francia, nel Regno Unito e successivamente anche in Italia e in Prussia, mentre la monarchia assoluta si conserva in Austria e in Russia. Il potere legislativo risulta strutturato secondo un modello a due livelli, nel quale una camera alta o Senato, i cui componenti sono designati dal potere esecutivo, fronteggia, esercitando una funzione di filtro o di decantazione, una camera bassa in cui siedono deputati espressi dall'elettorato.

La reale trionfatrice della stagione rivoluzionaria fu senz'altro la classe borghese che, quasi completamente priva di influenza politica durante l'assolutismo, si affermò come classe politicamente egemone nel contesto dei nuovi Stati liberali. Il successo della borghesia inaugura una nuova vicenda storica e politica, che avrà come tema l'allargamento del suffragio, inizialmente ristretto secondo rigorosi criterî censitari. Le frequenti sollevazioni che percorreranno l'Ottocento avranno come obiettivo, quindi, quello di sottrarre alla borghesia il controllo del potere legislativo, portando alla ribalta le problematiche e le aspirazioni di un nuovo soggetto politico, il proletariato.

Negli Stati liberali, la dimensione della salvaguardia dei diritti individuali dell'arbitrio dei governanti - dimensione che rappresenta uno dei lasciti della rivoluzione francese - prevale sull'istanza democratica. $\mathrm{Ne}$ emerge un modello di cittadino che è esemplato sul proprietario borghese, difeso dall'invadenza del potere esecutivo da una sfera di diritti che si vogliono naturali ma che, in sostanza, sono definiti dalla legge (prodotta dalle assemblee legislative, a loro volta espressione degli interessi della borghesia). Al tempo stesso, emergono diverse maniere di ricostruire il significato dell'esperienze rivoluzionaria alla luce del nuovo contesto politico e sociale.

\section{Constant e Tocqueville}

In Francia l'esordio del XIX secolo è segnato dagli scritti controrivoluzionari di autori come Joseph de Maistre, Louis-Gabriel Ambrose de Bonald e Félicité-Robert de Lamennais. Tuttavia, accanto al pensiero controrivoluzionario fioriscono anche altri tentativi di ripensare la vicenda della rivoluzione. A questo proposito occorre ricordare il contributo di Benjamin Constant (1760-1825). Constant ritiene che il principio della sovranità popolare sia ormai irrinunciabile. Tuttavia, per evitare gli eccessi

2011. Per una disamina delle soluzioni istituzionali, cfr. D. Novarese, Le istituzioni politiche della Restaurazione, in Meriggi, Tedoldi (a cura di), Storia delle istituzioni politiche, cit., pp. 103-127. 
del Terrore rivoluzionario diventa necessario concepire dei limiti entro i quali la potestà legislativa deve essere contenuta affinché non si traduca nell'esercizio di un potere illimitato.

Tali limiti sono costituiti dai diritti inviolabili degli individui che, sanciti da una legge generale e astratta, definiscono una sfera privata inaccessibile al potere politico. Secondo Constant ne deriva che la libertà dei moderni è differente dalla libertà degli antichi: per questi ultimi la libertà consisteva in una forma di partecipazione, quale quella che si poteva ritrovare nell'Atene democratica. Per i moderni, viceversa, la libertà consiste in una forma di protezione dallo Stato. In questo modo, Constant distingueva implicitamente l'esperienza repubblicana della seconda fase rivoluzionaria dall'impulso iniziale, compatibile con una traduzione e implementazione liberale ${ }^{12}$.

Nel solco del liberalismo si colloca anche Alexis de Tocqueville $(1805-1859)^{13}$. Tocqueville è un autore nodale per la riflessione sulla democrazia nell'Ottocento. Anzi, si può dire che con la sua opera $L a$ democrazia in America, scritta dopo un lungo soggiorno negli Stati Uniti, la democrazia e le sue peculiari problematiche diventano un tema centrale del pensiero politico. Da notare che con la parola 'democrazia' Tocqueville si riferisce alla democrazia rappresentativa moderna, congedandosi dalla tradizione, ancora viva al tempo delle rivoluzioni americana e francese, secondo la quale democratiche in senso proprio erano soltanto le città-Stato, mentre nel caso degli Stati rappresentativi contemporanei si doveva parlare di repubbliche.

Il punto di partenza di Tocqueville è dato dalla convinzione che la 'rivoluzione democratica' sia il punto di approdo di una vicenda secolare - in cui la monarchia assoluta aveva esercitato un ruolo centrale - di erosione delle differenze di status in direzione dell'uguaglianza delle condizioni. Negli Stati moderni l'articolazione in ceti lascia il posto all'uguaglianza di fronte alla legge e lo spirito democratico alimenta una vera e propria passione per l'uguaglianza. La democrazia, quindi, non è più una forma di governo fra le altre: è un modo in cui si struttura la società. Al tempo stesso, questo processo secolare ha visto l'ininterrotto rafforzamento del potere centrale, che adesso si contrappone direttamente alla molteplicità

${ }^{12}$ Su Constant, cfr. M. Barberis, Benjamin Constant. Rivoluzione, costituzione, progresso, il Mulino, Bologna 1988. In traduzione italiana, si possono vedere: B. Constant, Principî di politica (ed. or. 1815), a cura di di U. Cerroni, Editori Riuniti, Roma 1970; Id., La libertà degli antichi, paragonata a quella dei moderni (ed. or. 1819), a cura di G. Paoletti, Einaudi, Torino 2005.

${ }_{13} \mathrm{Su}$ Tocqueville, cfr.: Ch. Welch, de Tocqueville, Oxford University Press, Oxford 1999; F.M. De Sanctis, Tocqueville: democrazia e rivoluzione, Editoriale Scientifica, Napoli 2000; L. Jaume, Tocqueville. Les sources aristocratiques de la liberté, Fayard, Paris 2008; L. Re, Il liberalismo coloniale di Alexis de Tocqueville, Giappichelli, Torino 2012. 
degli individui, strappati ai legami comunitari che in passato li nascondevano all'occhio del sovrano ${ }^{14}$.

Ciò comporta una nuova mobilità sociale - anche individui di nascita umile possono arricchirsi - ma comporta anche la tendenza verso un individualismo sempre più sfrenato - caratteristica decisiva della società americana - e l'assenza di quelle strutture che, pur nell'ambito di un ordine fondato sul privilegio, garantivano una certa misura di libertà. Il tratto caratterizzante dell'antropologia tocquevilleana è dato dal fatto che la passione per l'uguaglianza si traduce in impulso acquisitivo volto al perseguimento di piaceri che accrescono la separazione fra gli individui, imponendo modelli di riferimento esteriori ${ }^{15}$.

In definitiva, l'individualismo esercita una pressione verso la mediocrità e il conformismo. La stessa democrazia rischia allora di trasformarsi in una tirannide della maggioranza, in sostanza in una forma di governo nella quale i non proprietari - negli Stati Uniti, a differenza che negli Stati europei, non sussistevano limiti censitari per l'ammissione al diritto di voto -, che sono in numero maggiore dei proprietari, governano per mano di deputati sempre più sensibili all'opinione popolare ${ }^{16}$. Questa pressione, però, è controbilanciata in America dai fattori che incentivano il legame sociale: il pluralismo, esaltato dall'articolazione federale; l'istituzione della giuria ${ }^{17}$; la partecipazione civica; l'associazionismo ${ }^{18}$. È la pratica dell'autogoverno, inscritta nell'ethos dei coloni americani, a schermarli dalle tendenze espansive del potere centrale, controbilanciando la spinta conformizzante ${ }^{19}$.

Ciò che sarebbe necessario per scongiurare la deriva dispotica insita nella democrazia è una sorta di 'ispessimento' dei nuovi rapporti che hanno preso il posto della vecchia articolazione cetuale. Proprio ciò che sembra mancare in Europa, dove invece si assiste a una polverizzazione del tessuto sociale cui fa da contraltare l'accrescimento dei poteri della macchina statale ${ }^{20}$. Il risultato è una mole sempre crescente di leggi che invadono gli spazi di autonomia, con l'obiettivo di smussare i profili in-

14 È il tema portante della seconda grande opera di Tocqueville, L'antico regime e la rivoluzione (ed. or. 1856), in Id., Scritti politici, vol. I, a cura di N. Matteucci, Utet, Torino 1969.

${ }^{15}$ Sull'antropologia di Tocqueville, cfr. E. Pulcini, Lïndividuo senza passioni. Individualismo moderno e perdita del legame sociale, Boringhieri, Torino 2001, pp. 127 sgg.

${ }^{16}$ Vedi A. de Tocqueville, La democrazia in America (ed. or. 1835-40), a cura di M. Tesini, 2 voll., Città Aperta, Troina 2005, vol. I, parte II, cap. 7, pp. 295-313.

${ }^{17}$ Ivi, vol. I, parte II, cap. 8, pp. 314-332.

${ }^{18}$ Ivi, vol. II, parte II, capp. 4-5, pp. 125-135.

${ }^{19}$ Ivi, vol. I, parte I, cap. 5, pp. 100-113.

${ }^{20}$ Cfr. anche A. de Tocqueville, Il pauperismo, a cura di M. Tesini, Edizioni Lavoro, Roma 1998. 
dividuali, rendendo i soggetti disciplinati ${ }^{21}$. L'apparato politico e amministrativo genera, allora, una nuova forma di dispotismo, un dispotismo mite, non più fondato sull'imposizione che viene dall'alto, ma su un'opera capillare di ottundimento delle passioni pubbliche, di svuotamento dei tratti individuali e di soddisfacimento dei «piaceri piccoli e volgari» che assopiscono le masse $\mathrm{e}^{22}$.

In questo modo, Tocqueville coglie perfettamente statu nascenti le assunzioni implicite della democrazia liberale, individuando il nesso profondo che lega istituzioni e società. Se tali assunzioni non sono soddisfatte, viene meno la possibilità di pensare all'esistenza di una volontà generale, preordinata al perseguimento del bene comune, cui si sostituisce la sommatoria degli impulsi acquisitivi degli elettori.

\section{Bentham e John Stuart Mill}

Nel Regno Unito il pensiero politico della restaurazione si salda alla tradizione illuminista precedente. E la peculiare espressione dell'illuminismo inglese è data dall'utilitarismo, corrente di cui Jeremy Bentham (1748-1832) è comunemente identificato come il primo esponente ${ }^{23}$.

Secondo l'approccio utilitarista le norme pratiche - etiche e giuridiche - devono essere scelte in modo da massimizzare l'utilità - concepita come una quantità misurabile di piacere - dei destinatari. Questo comporta un generale ripensamento del compito delle istituzioni. Per esempio, Bentham sostiene che, in ambito giuridico, l'Inghilterra debba abbandonare la secolare tradizione di common law per darsi leggi scritte, razionalmente formulate in modo da produrre le conseguenze più «felici» ${ }^{24}$.

L'eredità benthamiana si ritrova nel maggior pensatore politico inglese dell'Ottocento, John Stuart Mill (1806-1873) ${ }^{25}$. Mill condivide l'impostazione utilitarista che aveva assimilato, oltre che da Bentham,

${ }^{21}$ A questo proposito cfr. anche gli scritti che Tocqueville dedica alla riforma del sistema carcerario: A. de Tocqueville, Scritti penitenziari, a cura di L. Re, Edizioni di Storia e Letteratura, Roma 2002.

${ }^{22}$ Vedi Tocqueville, La democrazia in America, cit., vol. II, parte IV, capp. 5-7, pp. 360-390.

${ }^{23}$ Sul pensiero politico di Bentham, cfr. Ph. Schofield, Utility and Democracy: The Political Thought of Jeremy Bentham, Oxford University Press, New York 2006.

${ }^{24}$ Cfr.: J. Bentham, Un frammento sul governo (ed. or. 1766), a cura di S. Marcucci, Giuffrè, Milano 1990; Id., Introduzione ai principî della morale e della legislazione (ed. or. 1789), a cura di E. Lecaldano, Utet, Torino 1998.

${ }^{25} \mathrm{Su}$ Mill politico, cfr.: N. Urbinati, A. Zakaras (eds.), J.S. Mill's Political Thought: A Bicentennial Reassessment, Cambridge University Press, Cambridge 2007; D. Miller, J.S. Mill: Moral, Social, and Political Thought, Polity Press, Cambridge 2010; D. Brink, Mill's Progressive Principles, Clarendon Press, Oxford 2013. 
da suo padre James Mill che di Bentham era stato collaboratore. Per tale motivo, ritiene che la produzione legislativa dovrebbe essere improntata a criteri di razionalità: le leggi dovrebbero essere stese da un'apposita Commissione legislativa e approvate da un Parlamento eletto secondo criteri rappresentativi ${ }^{26}$. Quanto al voto, Mill ritiene che esso spetti a tutti i cittadini che pagano le tasse, incluse le donne, e che i rappresentanti debbano essere investiti di un mandato libero ${ }^{27}$.

$\mathrm{Al}$ tempo stesso, avendo letto attentamente Tocqueville ${ }^{28}$, Mill si mostra particolarmente sensibile ai pericoli degenerativi cui sono esposte le società democratiche. Inoltre, è convinto che la partecipazione politica debba essere coniugata a un'attenta educazione delle masse e che una condizione essenziale della democrazia risieda nell'equilibrio fra apertura verso tutte le istanze e valutazione competente delle conseguenze delle scelte politiche. Per questo motivo, sostiene anche che il principio rappresentativo vada corretto allo scopo di scongiurare il pericolo di una legislazione di classe attribuendo un peso differenziato ai voti espressi ai membri di determinati gruppi sociali ${ }^{29}$.

L'obiettivo è quello di permettere alle persone «dotate di qualità superiori» di esercitare un'influenza maggiore. Tuttavia, in prima approssimazione, il voto verrà quantificato sulla base della ricchezza ${ }^{30}$. Quindi, in definitiva, il liberalismo utilitarista di Mill - che si esprime anche nella sua nota teoria della libertà come autonomia, che può essere limitata solo per impedire a un individuo di nuocere agli altri ${ }^{31}$ - non è troppo differente dal liberalismo mainstream - da Locke a Constant che auspicava l'adozione di un criterio censitario nella determinazione del diritto di voto e si focalizzava sulla difesa dei diritti individuali dalle interferenze da parte dello Stato.

\section{Kant}

Negli Stati di area tedesca prevale nel secondo Settecento una versione dell'illuminismo secondo la quale il progresso politico e sociale deve avvenire non attraverso una frattura rivoluzionaria e una completa renovatio della società ma secondando l'attività riformatrice di un so-

${ }^{26}$ Vedi J.S. Mill, Considerazioni sul governo rappresentativo (ed. or. 1861), a cura di M. Prospero, Editori Riuniti, Roma 1997, cap. 5, pp. 71-88.

${ }^{27}$ Ivi, cap. 8, pp. 127-144.

${ }^{28}$ I saggi di Mill su Tocqueville sono raccolti in J.S. Mill, L'America e la democrazia, a cura di P. Adamo, Bompiani, Milano 2005.

${ }^{29}$ Vedi Mill, Considerazioni sul governo rappresentativo, cit., pp. $134 \mathrm{sgg}$.

${ }^{30}$ Ivi, pp. 135-136.

${ }^{31}$ Cfr. J.S. Mill, Sulla libertà (ed. or. 1859), a cura di G. Mollica, Bompiani, Milano 2000. 
vrano illuminato, come nell'esempio di Federico II di Prussia. Questo atteggiamento si ripercuoterà sulla valutazione della rivoluzione francese, che, pur inizialmente salutata come affermazione degli ideali di riforma, verrà poi fermamente respinta nel momento in cui sfocerà nel Terrore.

Questi motivi sono presenti in Immanuel Kant (1724-1804), il più importante pensatore illuminista tedesco e uno dei più grandi filosofi di tutti i tempi ${ }^{32}$. Kant ritiene che le leggi dello Stato siano dirette unicamente a costringere il comportamento esterno degli individui allo scopo di rendere possibile l'armonizzazione delle condotte, a contenere le propensioni egoistiche che ostacolano la naturale socievolezza umana, senza incidere sulla sfera interna, che invece è dominio della morale ${ }^{33}$. A questo principio si collegano la libertà religiosa e il diritto di manifestare liberamente la propria opinione.

Per quanto riguarda la fondazione del potere politico, Kant si inserisce nella tradizione contrattualista, ritenendo hobbesianamente che sia necessario un passaggio «artificiale» che consenta di trasformare l'originario stato di guerra in una condizione pacifica. D'altra parte, in continuità con l'insegnamento di Locke, lo stato prepolitico non è una condizione non-giuridica, perché in esso sono già presenti rapporti di diritto privato fondati su leggi razionali che regolano relazioni di tipo economico. Tali rapporti sono ancora provvisori, a causa dell'assenza di strutture coattive; ne segue che, per renderli definitivi, si rende necessario uscire dallo stato di natura. Per questa ragione, la nozione kantiana di contratto è affatto peculiare: per Kant il contratto è un'idea della ragione, non un fatto, un accordo empirico che potrebbe aver avuto luogo nel passato, ma una sorta di espediente razionale per chiarire il passaggio da forme di convivenza prepolitiche a forme di convivenza regolate dal diritto. Non un evento storico, ma un principio normativo che sancisce la doverosità dell'uscita dallo stato di natura. Ciò implica anche che l'elemento della volontà non sia centrale come negli altri pensatori contrattualisti, mentre emerge piuttosto il principio del dover essere razionale ${ }^{34}$.

L'impronta razionale è all'opera anche nel definire la relazione tra governanti e governati. Per un verso, infatti, Kant distingue tra Stato di diritto, che ha forma repubblicana, e Stato dispotico, caratterizzato dall'arbitrio.

${ }^{32}$ Su Kant politico, cfr.: W. Kersting, Wohlgeordnete Freiheit. Immanuel Kants Rechts- und Staatsphilosophie, de Gruyter, Berlin 1984; F. Gonnelli, La filosofia politica di Kant, Laterza, Roma-Bari 1996; A. Ripstein, Force and Freedom: Kant's Legal and Political Philosophy, Harvard University Press, Cambridge, Mass. 2009.

33 Vedi I. Kant, Metafisica dei costumi (ed. or. 1797), a cura di G. Landolfi Petrone, Bompiani, Milano 2006, «Introduzione alla Metafisica dei costumi», I, pp. 27-29.

34 Vedi I. Kant, Sopra il detto comune: «questo può essere giusto in teoria ma non vale per la pratica» (ed. or. 1793), in Id., Scritti politici e filosofia della storia e del diritto, a cura di G. Solari et al., Utet, Torino 1956, p. 262. 
Lo Stato di diritto è fondato sulla libertà, l'uguaglianza e l'autonomia dei cittadini. La libertà dei cittadini comporta l'illegittimità del governo paternalistico. Nello Stato di diritto il popolo è detentore della sovranità, che viene espressa attraverso la rappresentanza politica e che si estrinseca nel potere legislativo ${ }^{35}$. Il contratto che segna l'uscita dallo stato di natura vincola anche il legislatore a operare come se autore della legge fosse l'intero corpo politico. Per parte propria, il corpo politico è tenuto a prestare il proprio assenso a una legge che sia espressione della ragione. In questo modo, l'architettura dello Stato kantiano tiene unite ragione e volontà. Per quanto concerne il potere esecutivo, esso è detenuto da un reggitore - per questo motivo anche una monarchia configura un regime repubblicano, nel senso di Kant. Il potere giudiziario, infine, è indipendente ${ }^{36}$.

Il razionalismo kantiano implica che la democrazia sia considerata in maniera ambivalente: da un lato Kant ritiene che l'esercizio della sovranità popolare possa avvenire in forme rappresentative ${ }^{37}$ - per quanto la rappresentanza che Kant ha in mente contempli la distinzione, già introdotta da Sieyès, tra cittadini attivi e passivi ${ }^{38}$-, dall'altro, la democrazia degenera in dispotismo - qui chiaramente Kant aveva presente l'esperienza del Terrore rivoluzionario - nel momento in cui viene vanificata la separazione dei poteri e il legislatore si identifica con il reggitore ${ }^{39}$. In pratica, è l'espediente 'formale' della rappresentanza che, introducendo una distanza tra volontà politica e volontà empirica, ne consente la razionalizzazione.

La negazione kantiana del diritto di resistenza non può che confermare questa impostazione. Infatti, a differenza di quanto aveva sostenuto Locke, persino contro un sovrano che abusi del proprio potere, non è configurabile in capo ai sudditi un diritto di resistenza, perché nel momento in cui si apre una guerra civile che oppone i sostenitori del re ai suoi oppositori non è possibile individuare alcuna autorità legittimata a decidere il conflitto. La resistenza contro il sovrano è quindi un evento che non può essere integrato nell'architettura razionale della politica ${ }^{40}$.

\section{Fichte e Hegel}

Come si è visto, l'impostazione razionalistica kantiana influenza profondamente la valutazione degli eventi contemporanei, rendendo inevita-

35 Ivi, pp. 254 sgg.

${ }^{36}$ Vedi Kant, Metafisica dei costumi, cit., Dottrina del diritto, parte II, sez. I, 43-49, pp. 229-243.

${ }^{37}$ Ivi, Dottrina del diritto, parte II, sez. I, 52, pp. 291-295.

${ }^{38}$ Ivi, Dottrina del diritto, parte II, sez. I, 46, pp. 235-237.

39 Vedi I. Kant, Per la pace perpetua (ed. or. 1795), in Id., Scritti politici, cit., pp. 294-297.

40 Vedi Kant, Sopra il detto comune, cit., pp. 263-266. 
bile una presa di distanza dall'esito della rivoluzione, pur in presenza di una sincera condivisione dei suoi ideali ispiratori. Questo tipo di reazione si ritrova anche negli scritti del filosofo che dell'opera di Kant si era proposto di diventare il continuatore, Johann Gottlieb Fichte (1762-1814). Fichte, infatti, sostiene la legittimità teorica della rivoluzione francese, ma ritiene anche che la democrazia diretta si risolva, in sostanza, in una forma di violenza e che l'unica forma di governo che garantisca la libertà sia quella basata sulla rappresentanza ${ }^{41}$.

In questa fase, la proposta fichtiana si distingue da quella kantiana per il fatto di respingere il principio di separazione dei poteri e per una originale innovazione istituzionale: l'eforato, una magistratura elettiva con il compito di controllare l'azione dei governanti. Questa innovazione è importante perché conferma che la partecipazione popolare alle attività di governo richiede un filtro e una mediazione, assicurata anche dal fatto che gli efori devono essere scelti fra i cittadini più dotti ${ }^{42}$.

Nel decennio seguente, tuttavia, l'esperienza della dittatura napoleonica e poi dell'occupazione della Prussia, successiva alla battaglia di Jena, spingerà Fichte ad accentuare il profilo nazionalistico della sua riflessione. Ciò determina una progressiva rivalutazione dell'articolazione interna alla società, di cui lo Stato deve farsi organizzatore e garante, oltrepassando quindi il ruolo di mero regolatore degli arbitrî esterni che la rilettura kantiana del contrattualismo gli assegnava, e una maggiore enfasi sulla specificità culturale tedesca vista in rapporto alla realizzazione del destino umano di libertà 43 .

L'itinerario intellettuale di Fichte procede dal problema di 'fare i conti' con la rivoluzione francese in direzione di una sostanziale liquidazione dell'impianto individualista del contrattualismo e della riscoperta della 'sostanza' culturale della società. Questo percorso è ancora più evidente in Georg Wilhelm Friedrich Hegel (1770-1831) ${ }^{44}$.

${ }^{41}$ Cfr. J.G. Fichte, Contributo per rettificare i giudizi del pubblico sulla rivoluzione francese (ed. or. 1793), in Id., Sulla rivoluzione francese, a cura di V.E. Alfieri, Laterza, Roma-Bari 1974. Sul pensiero politico di Fichte, cfr. C. De Pascale, Etica e diritto. La filosofia pratica di Fichte e le sue ascendenze kantiane, il Mulino, Bologna 1995 .

${ }_{42}$ Vedi J.G. Fichte, Fondamenti del diritto naturale (ed. or. 1796), a cura di L. Fonnesu, Laterza, Roma-Bari 1994, parte I, sez. III, cap. 3, pp. 135-165. Cfr. anche G. Rametta, Politica e democrazia nell'idealismo tedesco, in Duso (a cura di), Oltre la democrazia, cit., pp. 175-208: 191-192.

${ }_{43}$ Cfr. J.G. Fichte, Discorsi alla nazione tedesca (ed. or. 1807-1808), a cura di G. Rametta, Laterza, Roma-Bari 2003.

${ }_{44}$ Su Hegel politico, cfr.: Sh. Avineri, La teoria hegeliana dello Stato (ed. or. 1972), Laterza, Roma-Bari 1973; C. Cesa, Hegel filosofo politico, Guida, Napoli 1976; G. Preterossi, I luoghi della politica. Figure istituzionali della filosofia del diritto hegeliana, Guerini e Associati, Milano 1992; Th. Brooks, Hegel's Political Philoso- 
Hegel ritiene che il razionalismo moderno abbia condotto a un completo fraintendimento del fondamento del potere politico. Lo Stato non è il risultato di una costruzione artificiale. Di qui il rifiuto del contrattualismo che già Kant aveva contribuito a screditare: se lo Stato già non esistesse, l'accordo dei singoli non potrebbe istituirlo. La concezione contrattualista, infatti, è basata sull'incomprensione della nozione di libertà che non può esaurirsi nella capacità di concludere negozî giuridici o di autodeterminarsi individualmente ma rimanda al tessuto di relazioni in cui $\mathrm{i}$ soggetti sono naturalmente immersi ${ }^{45}$.

Per Hegel lo Stato è una totalità organica in cui il tutto è superiore alle parti. Lo Stato moderno è il frutto di processi storici di emancipazione sociale: dalla polis alla codificazione romana, al mondo feudale, fino alla modernità e alla rivoluzione. La consapevolezza di questa dimensione storica è un elemento imprescindibile nella valutazione degli eventi e delle istituzioni. L'esperienza della rivoluzione francese lascia intravedere la dimensione astratta e autodistruttiva dell'idea di sovranità popolare. Nel periodo del Terrore si assiste allo scatenamento di una nozione di libertà così astratta da non tollerare alcuna differenziazione al suo interno, traducendosi nell'apoteosi della morte ${ }^{46}$. $\mathrm{Al}$ contrario, la vera libertà risiede in un ordinamento concreto, nel quale l'assetto costituzionale è il portato di un'articolazione sociale sottostante. Per questo motivo, la vera libertà, intesa come possibilità di essere inseriti in un sistema di rapporti sociali oggettivi che consentano l'autorealizzazione, può aversi soltanto nel contesto degli Stati moderni. Lo Stato rappresenta una totalità costituzionale già in atto, un'idea etica che si manifesta attraverso la mediazione della società civile. Per questo motivo, l'astratta concezione di un popolo indifferenziato detentore della sovranità è insoddisfacente.

Diversamente dai contrattualisti, che ponevano la società civile come esito di un artificio - il patto - prima del quale non esisteva propriamente il popolo, Hegel, nella costruzione della Filosofia del diritto, colloca la società civile come uno stadio intermedio fra la famiglia e lo Stato - in ciò recuperando la lezione della Politica di Aristotele. Più precisamente, la società civile corrisponde - e qui forse si può avvertire un'eco della concezione kantiana secondo la quale il contratto politico è preceduto da forme di coesistenza prepolitica - al sistema delle relazioni coordinate da strumenti di diritto privato, su cui si innestano le prime forme di amministrazione dirette a governare le disuguaglianze generate dalle

phy: A Systematic Reading of the Philosophy of Right, Edinburgh University Press, Edinburgh 2012.

45 Vedi G.W.F. Hegel, Le maniere scientifiche di trattare il diritto naturale (ed. or. 1803), a cura di C. Sabbatini, Bompiani, Milano 2016, pp. 305-306, 399-401.

${ }^{46}$ Vedi G.W.F. Hegel, Fenomenologia dello spirito (ed. or. 1807), a cura. di V. Cicero, Rusconi, Milano 1995, VI, B, III, pp. 783-799. 
dinamiche economiche e la regolamentazione delle corporazioni ${ }^{47}$. Invece, lo Stato in senso proprio è rappresentato unicamente da quelle modalità di associazione politica nelle quali la libertà soggettiva si manifesta concretamente nella fisionomia delle istituzioni. Lo Stato ricapitola in sé le forme di associazione precedenti. Per questo Hegel ritiene che lo Stato, che assume la veste di una moderna monarchia costituzionale, in cui vige la separazione dei poteri, debba al contempo conservare traccia della propria articolazione in ceti e che questi ultimi debbano trovare una forma di rappresentanza nelle strutture statuali ${ }^{48}$.

Ciò comporta anche la liquidazione delle categorie filosofico-politiche della modernità. Categorie come «sovranità popolare», «popolo», «repubblica» e «democrazia» vengono analizzate fino a rivelarne le contraddizioni interne ${ }^{49}$. Emerge, così, che l'intero discorso democratico si regge su una finzione insostenibile, quella della volontà generale, che condivide le stesse ambiguità della nozione di popolo: come il popolo non possiede un'identità autonoma prima del suo esistere all'interno dello Stato, ma è concepibile soltanto come «massa amorfa», così la volontà dei molti può diventare volontà comune solo per tramite delle istituzioni.

\section{Marx}

La concezione hegeliana della società civile come prius rispetto allo Stato, la cui fisionomia è plasmata dai rapporti di lavoro e di produzione è lo sfondo imprescindibile su cui si staglia il pensiero politico di Karl Marx $(1818-1883)^{50}$. Da Hegel, Marx eredita anzitutto la convinzione che dello Stato non si può parlare prescindendo dalla dimensione 'sostanziale' dei rapporti che hanno luogo all'interno della società civile. Anche per Marx, come per Hegel, l'astrazione contrattualista che ricollegava la nascita dello Stato all'accordo delle volontà individuali è insostenibile. Per questo l'armamentario concettuale del pensiero liberale va ricondotto al contesto socioeconomico che lo ha generato ${ }^{51}$. Al tempo stesso, la direzione della riflessione marxiana è molto diversa da quella seguita da Hegel e ci sottopone un modo di reagire all'ascesa dello Stato borghese dell'Ottocento

${ }^{47}$ Sul tema della società civile in Hegel, cfr. G. Cesarale, La mediazione che sparisce. La società civile in Hegel, Carocci, Roma 2009.

${ }^{48}$ Vedi G.W.F. Hegel, Lineamenti di filosofia del diritto (ed. or. 1821), a cura di G. Marini, Laterza, Roma-Bari 1987, parte III, sez. III, A, I, c, 301-311, pp. 240-249.

${ }^{49}$ Ivi, parte III, sez. III, A, I, a, 279, pp. 224-227.

${ }^{5} \mathrm{Su}$ Marx, si possono vedere: Sh. Avineri, Il pensiero politico e sociale di Karl Marx (ed. or. 1968), il Mulino, Bologna 1972; A. Wood, Karl Marx, Routledge, London 2004; T. Carver (ed.), The Cambridge Companion to Marx, Cambridge University Press, Cambridge 2008; S. Petrucciani, Marx, Carocci, Roma 2009.

${ }^{51}$ Cfr. K. Marx, Sulla questione ebraica (ed. or. 1843), a cura di D. Fusaro, Bompiani, Milano 2007. 
opposto rispetto al pensiero liberale. Marx, infatti, teorizza la necessità di una trasformazione complessiva della società e della politica nel segno di un ribaltamento dei rapporti di forza tipici dello Stato borghese.

Il punto di partenza è la concezione della società civile, che Marx deriva da Hegel. La società civile rappresenta una sorta di nuovo stato di natura, teatro di conflitti di tipo economico. In sostanza, alla guerra perpetua tra individui diagnosticata da Hobbes, Marx, sviluppando le scarne notazioni hegeliane della Filosofia del diritto ${ }^{52}$, sostituisce la lotta fra le classi. Questo conflitto ha un'origine «naturale», in quanto è radicato in dinamiche spontanee, ma ha anche una coloritura politica, in quanto gli assetti giuridici e politici che vigono nella società sono un prodotto, una sovrastruttura, rispetto alla struttura economica sottostante. Si genera quindi una frattura fra le profonde disuguaglianze economiche e l'uguaglianza formale vigente sul piano giuridico e politico.

Per questo motivo, lo scollamento tra società civile e Stato non può essere colmato attraverso le forme di rappresentanza organica di derivazione cetuale immaginate da Hegel, che ripropongono una logica premoderna, né confidando nel rafforzamento degli strumenti democratici - es. nell'ampliamento del suffragio, come pure Marx aveva inizialmente pensato ${ }^{53}$. Questo perché le strutture dello Stato liberale sono state plasmate dai rapporti di forza economici, che vedono il predominio della classe borghese; pertanto, la condizione del proletariato non può essere modificata se non scardinando quei rapporti di forza e instaurando assetti politici nuovi, vale a dire rimuovendo l'antitesi stessa tra società civile e Stato. In ultima analisi, la direzione che Marx addita è quella di una presa del potere da parte della classe operaia, che successivamente darà luogo a una società pacificata, senza classi, senza proprietà privata e senza Stato ${ }^{54}$.

Ma se l'approdo finale del percorso immaginato da Marx dovrà essere una società senza Stato, non è immediatamente comprensibile quali debbano essere le tappe intermedie che conducono a questo esito ${ }^{55}$. A questo proposito, non è semplice estrarre dagli scritti di Marx indicazioni chiare. Si può tuttavia ricordare che, in alcuni contributi dell'ultima fase del suo pensiero, Marx additava le soluzioni istituzionali elaborate durante il periodo della Comune di Parigi, nel 1870, come un modello cui ispirarsi per

${ }^{52}$ Cfr. Hegel, Lineamenti di filosofia del diritto, cit., parte III, sez. II, C, a, 243245, pp. 187-189.

${ }_{53}$ Cfr. K. Marx, Critica della filosofia hegeliana del diritto pubblico (ed. or. 1843), a cura di M. Prospero, Editori Riuniti, Roma 2016. Sull'interpretazione marxiana di Hegel, cfr. Sh. Avineri, Il pensiero politico e sociale di Karl Marx, cap. 1.

${ }_{54}$ Cfr. K. Marx, F. Engels, Il manifesto del partito comunista (ed. or. 1847), a cura di B. Bongiovanni, Einaudi, Torino 2014.

55 Il tema è stato al centro di un vastissimo dibattito fra gli autori che si legano all'eredità marxiana. Cfr. D. Zolo, La teoria comunista dell'estinzione dello Stato, De Donato, Bari 1974. 
sovvertire le logiche dello Stato liberale ${ }^{56}$. Dunque, sostanzialmente una forma di democrazia 'rousseauiana', in cui delegati revocabili, vincolati dalle istruzioni della base che li ha designati, rendono possibile l'espressione della volontà generale, azzerando, in sostanza, la mediazione delle istituzioni politiche, sociali, economiche ${ }^{57}$.

$\mathrm{Al}$ di là delle controversie interpretative, è chiaro che Marx, per sfuggire al paradigma liberal-rappresentativo, considerato insostenibile, si rifugia nella duplice utopia di un mondo pacificato, cui fa da premessa la riattualizzazione della democrazia diretta. Qui, si potrebbe obiettare che, per rendere possibile la democrazia diretta, bisognerebbe mettere fuori gioco l'operatività di tutte le strutture socioeconomiche intermedie rispetto alla partecipazione politica degli individui. Ma questo risultato potrebbe darsi soltanto se lo Stato, inteso hegelianamente come totalità delle relazioni, fosse già estinto.

Da questo punto di vista, le considerazioni di Marx si contrappongono a quelle di Hegel e di Tocqueville, che avevano visto nella deriva giacobina della rivoluzione l'apogeo del dispotismo. Sul piano, invece, della critica dello Stato rappresentativo liberale le critiche marxiane si allineano a quelle dei suoi predecessori, lasciando un'eredità difficilmente eludibile alla riflessione successiva.

${ }^{56}$ Cfr. K. Marx, La guerra civile in Francia (ed. or. 1871), a cura di P. Togliatti, Editori Riuniti, Roma 1974.

57 Vedi anche l'interpretazione di Pietro Costa, che sottolinea la continuità con la critica giovanile della democrazia liberale. Vedi P. Costa, Civitas. Storia della cittadinanza in Europa, vol. 3, La civiltà liberale, Laterza, Roma-Bari 2001, pp. 275-276. 


\section{Capitolo quarto \\ Il Novecento}

\section{Tra Ottocento e Novecento}

Il XIX secolo in Europa è tutto punteggiato dall'esplosione di moti rivoluzionari di breve durata - i moti del 1820-21, quelli del 1830-31, i moti del 1848-49, le Guerre di Indipendenza in Grecia, Polonia e Italia, la rivolta di Parigi nel 1870. L'effetto complessivo di questi eventi sulle strutture dello Stato liberale è quello di un progressivo allargamento del suffragio, che per la prima volta diventa universale in molte realtà europee ${ }^{1}$.

L'allargamento del suffragio porta con sé altre profonde trasformazioni. La conseguenza più immediata è che il vecchio Stato monoclasse - in cui soltanto la classe borghese partecipava attivamente alle attività politiche - si trasforma in Stato pluriclasse. Come conseguenza, la stessa classe politica si rinnova con l'ingresso di figure tratte dalle professioni intellettuali, che con il passare del tempo sostituiscono i notabili provenienti dall'aristocrazia terriera. Questo avvicendamento riguarda, in primo luogo, le camere dei deputati; in progresso di tempo, tuttavia, anche le camere alte, originariamente composte da membri designati dal sovrano, diventeranno più simili alle camere basse $^{2}$. Il potere giudiziario accentua le proprie funzioni di garanzia. Tutte queste trasformazioni si ripercuotono anche sulle politiche pubbliche. L'apparato amministrativo si amplia, parallelamente all'aumento dei compiti dello Stato, non più impegnato soltanto nella difesa dei diritti dei proprietari ma sempre

${ }^{1}$ Su questa vicenda e, più in generale, sulle istituzioni del secondo Ottocento, vedi M. Meriggi, Dal liberalismo alla democrazia (1848-1914), in Meriggi, Tedoldi (a cura di), Storia delle istituzioni politiche, cit., pp. 129-148.

${ }^{2}$ Ivi, pp. 131 sgg.

Leonardo Marchettoni, Breve storia della democrazia: da Atene al populismo, ISBN 978-88-6453760-3 (print) ISBN 978-88-6453-761-o (online PDF) ISBN 978-88-6453-762-7 (online EPUB), (C) 2018 Firenze University Press 
più attivo nella sfera sociale: erogazione di prestazioni di assistenza pubblica, previdenza, istruzione, servizi ${ }^{3}$.

Nel contempo, e correlativamente, anche altri soggetti acquistano visibilità. Le associazioni operaie guadagnano rilevanza, soprattutto in Inghilterra, fino a divenire interlocutori delle amministrazioni nella negoziazione della disciplina sul lavoro. Si genera una nuova galassia di movimenti articolata intorno alle organizzazioni sindacali, che assumono i principî del socialismo marxista, declinandoli in chiave moderata e pragmatica.

Nel 1875 nasce il primo Partito moderno, il Partito Socialdemocratico tedesco, in cui diventa rapidamente prevalente l'ispirazione marxista, seguito da analoghe formazioni in Francia, in Italia (in cui il Partito Socialista nasce nel 1892) e in Inghilterra (con la fondazione del Partito Laburista nel 1900). Il Partito Socialdemocratico è il primo partito di massa, vale a dire il primo partito dotato di un'organizzazione stabile, radicato sul territorio, provvisto di funzionari professionalizzati, organizzato intorno a un programma che lo distingue dalle altre formazioni politiche e cui gli eletti devono attenersi ${ }^{4}$.

Nello stesso periodo aumenta la diffusione dei giornali quotidiani, grazie a innovazioni tecniche come la stampa in rotativa e il telegrafo. Questo fenomeno comporta la creazione di un nuovo spazio pubblico per la discussione e la diffusione di opinioni. Al contempo, l'espansione dei quotidiani segna anche una trasformazione profonda nelle loro caratteristiche: il quotidiano inizia a ospitare inserzioni pubblicitarie; nella sua gestione si fa strada una mentalità aziendalistica; in molti casi i quotidiani vengono controllati da grandi gruppi economici, potenzialmente interessati al contenuto delle notizie che vengono pubblicate ${ }^{5}$.

\section{Weber}

Negli anni a cavallo tra l'Ottocento e il primo Novecento si incomincia anche a riflettere sui mutati rapporti di forza in seno alla società. Come si è visto nel capitolo precedente, la riflessione di autori come Tocqueville e Marx aveva già messo in luce le aporie del modello liberale di democrazia. All'inizio del nuovo secolo queste aporie erano rese ancora più evidenti dall'allargamento del suffragio, dalle modificazioni del sistema politico e dall'ampliamento delle funzioni dell'apparato amministrativo.

3 Ivi, pp. 139 sgg. Sulle trasformazioni dell'amministrazione cfr. anche L. Mannori, B. Sordi, Storia del diritto amministrativo, Laterza, Roma-Bari 2013. Più in generale, sulla genesi della democrazia di massa, cfr. B. De Giovanni, Alle origini della democrazia di massa, Editoriale Scientifica, Napoli 2013.

4 Vedi D. della Porta, I partiti politici, il Mulino, Bologna 2015, cap. 2.

${ }^{5}$ Cfr. J. Habermas, Storia e critica dell'opinione pubblica (ed. or. 1962, 1990), a cura di M. Carpitella, Laterza, Roma-Bari 2002. 
Uno dei primi pensatori a riflettere criticamente su questo complesso di problemi è sicuramente Max Weber (1864-1920) ${ }^{6}$. Questa riflessione è direttamente collegata al modo in cui Weber ricostruisce la genesi del sistema sociale ed economico moderno. Attraverso la ricognizione diretta delle metamorfosi sociali connesse all'evoluzione dell'agricoltura prussiana, Weber matura una visione del capitalismo alternativa a quella marxiana, nella quale la dimensione 'valoriale' assume una rilevanza inedita'.

Weber nota come l'avvento del capitalismo non sia riconducibile unicamente al conflitto socioeconomico, ma sia connesso a un processo di autodisciplinamento da parte dei soggetti attraverso il lavoro, reso possibile dalla forma mentis imposta dalla religione protestante ${ }^{8}$. Tale processo è connesso alla razionalizzazione complessiva del mondo - cui Weber si riferisce con la locuzione «disincantamento del mondo» ${ }^{-}-$che travolge i legami personali connessi alle differenze di status sostituendo a essi un «cosmo oggettivo» di relazioni sociali coattive.

Il disincantamento del mondo comporta che vengano a mancare quadri di riferimento capaci di guidare le scelte individuali. In un mondo sempre più dominato dalla ragione tecnica e scientifica, si svuota lo stesso problema della ricerca di senso tramite il confronto tra le diverse visioni del mondo. Questa situazione si proietta anche sul piano politico, perché i valori liberali tradizionali non possono più essere giustificati in maniera indipendente, come si era cercato di fare nel passato: vengono meno le stesse precondizioni per ritenere che si diano leggi di condotta o principî dell'agire autonomamente fondati ${ }^{10}$.

In questa situazione, la difesa del liberalismo può avvenire solo su un piano procedurale, in ragione della sua importanza come strumento per permettere il confronto tra i diversi valori e le diverse visioni del mondo. Il meccanismo procedurale più raccomandabile è dato dalla democrazia rappresentativa. Weber, infatti, ritiene la democrazia diretta appropriata solo nel caso di gruppi sociali di estensione territoriale ridotta ed elevata

${ }^{6} \mathrm{Su}$ Weber, si possono vedere: W.J. Mommsen, Max Weber e la politica tedesca (ed. or. 1974), il Mulino, Bologna 1993; W. Scluchter, Il paradosso della razionalizzazione. Studi su Max Weber (ed. or. 1980), Liguori, Napoli 1987; D. Beetham, La teoria politica di Max Weber (ed. or. 1985), il Mulino, Bologna 1989; D. D’Andrea, L'incubo degli ultimi uomini. Etica e politica in Max Weber, Carocci, Roma 2005.

7 Cfr. gli scritti giovanili raccolti in M. Weber, Dalla terra alla fabbrica. Scritti sui lavoratori agricoli e lo Stato nazionale (1892-1897), a cura di F. Ferraresi e S. Mezzadra, Laterza, Roma-Bari 2005.

8 È questa la tesi portante dell'opera più nota di M. Weber, L'etica protestante e lo spirito del capitalismo (ed. or. 1904-1905), in Id., Sociologia delle religioni, 2 voll., a cura di C. Sebastiani, Utet, Torino 1976.

9 Vedi M. Weber, La scienza come professione (ed. or. 1919), a cura di P. Volonté, Rusconi, Milano 1997, p. 89.

${ }^{10}$ Vedi Beetham, La teoria politica di Max Weber, cit., pp. 10 sgg. 
omogeneità sociale, sia perché in questo caso non si rendono necessarie prestazioni amministrative articolate - che le istituzioni delle democrazie dirette non potrebbero assolvere -, sia perché soltanto queste condizioni sono compatibili con meccanismi di rappresentanza - che escludono la formazione di compromessi fra i rappresentanti ${ }^{11}$.

Le ricadute che questo processo proietta sugli assetti istituzionali sono amplissime. Riguardo allo Stato, Weber ritiene che le sue origini vadano di pari passo con l'ascesa del capitalismo. Lo Stato si definisce sulla base della capacità di monopolizzare l'uso legittimo della forza entro un determinato territorio ${ }^{12}$. Tale uso è legittimo in virtù del riferimento alla legge. Caratteristica dello Stato moderno è l'estensione dell'apparato amministrativo, organizzato secondo criteri di razionalità che lo apparentano in una certa misura al sistema produttivo capitalistico. L'amministrazione è affidata a un insieme di funzionari - la burocrazia - che rispondono alla legge in base al principio di legalità. L'operato di tali funzionari non è legittimato democraticamente ma in virtù della loro competenza tecnica ${ }^{13}$.

Lo Stato moderno si affida alle strutture burocratiche, che minacciano di espropriare la politica dai suoi compiti. L'espansione del dominio burocratico è irreversibile, tanto più in un contesto nel quale la domanda di coordinamento da parte del sistema economico e di prestazioni sociali da parte della cittadinanza è in costante aumento. Il rischio però è che questa espansione vada a restringere gli spazi di libertà soggettiva che ancora residuano, nel senso che gli imperativi tecnici che presiedono all'azione della burocrazia si pongono in contrasto con la possibilità di una scelta eticamente libera ${ }^{14}$.

Come rimedio all'eccessiva burocratizzazione dello Stato Weber, scartata l'opzione socialista che non risolverebbe il problema, ma anzi rischierebbe di condurre a un maggiore ampliamento dell'apparato amministrativo, auspica un rinnovamento e una nuova educazione politica della classe dirigente. Soprattutto, reputa necessario operare in direzione dell'irrobustimento delle istituzioni democratiche, in modo da accrescere

${ }^{11}$ Vedi M. Weber, Sistema elettorale e democrazia in Germania (ed. or. 1917), in Id., Scritti politici, introduzione di A. Bolaffi, trad. di A. Cariolato e E. Fongaro, Donzelli, Roma 1998, pp. 84 sgg. Cfr. anche la Sociologia del dominio nella nuova ricostruzione presentata in M. Weber, Economia e società. Dominio. L'economia in rapporto agli ordinamenti e alle forze sociali, a cura di M. Palma, Donzelli, Roma 2012.

${ }_{12}$ Vedi M. Weber, La politica come professione (ed. or. 1919), in Id., Scritti politici, cit., p. 178.

${ }^{13}$ Sui caratteri della razionalità burocratica, cfr. Beetham, La teoria politica di Max Weber, cit., cap. 3.

${ }^{14}$ Vedi D’Andrea, L’incubo degli ultimi uomini, cit., pp. $150 \mathrm{sgg}$. 
la rappresentatività del Parlamento. Quest'ultimo dovrebbe diventare l'organo nel quale si combatte la lotta tra i valori ${ }^{15}$.

Tuttavia, anche nel dibattito parlamentare la discussione sui valori deve misurarsi con l'invadenza delle logiche razionalistiche, rappresentate dall'organizzazione dei moderni partiti di massa. Le modificazioni del sistema politico conseguenti all'estensione del suffragio hanno reso i parlamenti inidonei a costituire una sede adeguata per il dibattito intorno alle scelte politiche, assegnando questo compito soprattutto ai partiti. I moderni partiti di massa, però, tendono a strutturarsi secondo principî burocratici che pregiudicano la loro attitudine a farsi interpreti della dimensione assiologica

Ma l'ascesa dei partiti non comporta necessariamente una deriva burocratica. Negli ultimi anni della sua vita, Weber riflette sulle caratteristiche che l'uomo politico deve possedere nell'epoca dei partiti di massa. Il partito di massa tende inevitabilmente a enfatizzare il ruolo del leader politico. A questo proposito, Weber sottolinea la rilevanza del carisma che contraddistingue il capo, capace di misurarsi con convinzione e responsabilità con i problemi reali, evitando la burocratizzazione della politica e, in definitiva, superando le secche di un modello di legittimazione legal-procedurale ${ }^{16}$.

La riflessione di Weber occupa una posizione nodale nella storia della democrazia. Weber, non solo mette perfettamente a fuoco le criticità che le trasformazioni della società e del sistema statuale pongono al modello politico liberale, ma arriva fino a cogliere l'insufficienza dell'interpretazione procedurale che pure aveva contribuito a delineare.

\section{La crisi della sovranità popolare e l'elitismo}

La conquista del suffragio universale aveva posto il problema del modo in cui l'immissione delle nuove masse avrebbe modificato gli equilibri democratici. In risposta, Weber aveva sostenuto che, per evitare la burocratizzazione della politica conseguente all'allargamento del suffragio è necessario investire sulle qualità soggettive dei leader politici. A questo problema la corrente che sarebbe stata detta dell'elitismo fornisce una risposta radicale: in tutti i sistemi democratici l'insieme di persone che tiene in mano le redini della politica è una ristretta minoranza. L'estensione del suffragio non sposta questo dato fondamentale, anzi lo rende

${ }_{15}$ Cfr. M. Weber, Parlamento e governo (ed. or. 1918), a cura di F. Fusillo, Laterza, Roma-Bari 1982. Cfr. anche Beetham, La teoria politica di Max Weber, cit., cap. 4.

${ }^{16}$ Vedi Weber, La politica come professione, cit. Cfr. anche: Mommsen, Max Weber e la politica tedesca, cit., pp. 293-338; Beetham, La teoria politica di Max Weber, cit., cap. 8; D’Andrea, L'incubo degli ultimi uomini, cit., pp. 236-49; F. Tuccari, Carisma e leadership nel pensiero di Max Weber, FrancoAngeli, Milano 1991, pp. 239-270. 
ancora più evidente. La minoranza che governa è una élite che dispone dei mezzi - economici, sociali, culturali - per esercitare il potere ${ }^{17}$.

Questo punto di vista viene esposto chiaramente da Gaetano Mosca (1858-1941), che sottolinea come il potere politico sia espressione del volere e degli interessi di una minoranza omogenea e organizzata - la classe politica ${ }^{18}$. Negli stessi anni l'economista Vilfredo Pareto (1848-1923) riflette sui cicli di riproduzione delle élite che assicurano il ricambio della classe dirigente e l'equilibrio sociale ${ }^{19}$. Infine, Robert Michels (1876-1936) diagnostica il nesso sottostante tra organizzazione e oligarchia all'interno dei sistemi democratici elaborando la «legge ferrea dell'oligarchia», che afferma che nelle democrazie moderne è inevitabile il rafforzamento dei partiti e dei gruppi ristretti che li dirigono ${ }^{20}$.

La demitizzazione elitista della democrazia costituisce la base della concezione procedurale elaborata da Joseph Alois Schumpeter (1883-1950) nelle sue opere principali e specialmente in Capitalismo, socialismo, democrazia. Per Schumpeter, che aveva una formazione da economista, la democrazia è un «accorgimento istituzionale» che permette a gruppi ristretti di acquisire il potere politico «mediante una competizione per il voto popolare ${ }^{21}$ : la democrazia è un metodo, un insieme di procedure che consente di produrre decisioni politiche, legittimandole tramite il riferimento alla volontà degli elettori. Pertanto, non c'è un nesso che vada dalla volontà popolare alle decisioni politiche di coloro che governano. Queste ultime sono completamente svincolate dalle scelte dei cittadini, ai quali, per contro, viene attribuito un ruolo limitato, esposti come sono alle lusinghe dei politici di professione e dei gruppi economici.

Questo punto si lega alla critica che Schumpeter svolge della «dottrina classica della democrazia», vale a dire la concezione secondo la quale la democrazia consiste in un insieme di accorgimenti istituzionali diretti a realizzare il bene comune attraverso l'operato di individui eletti che mettono in atto la volontà popolare ${ }^{22}$. Questa concezione, sostiene Schumpeter, possiede diversi punti deboli. In primo luogo, non esiste qualcosa come

${ }^{17}$ Sulla teoria delle élite, cfr. G. Parry, Le élites politiche (ed. or. 1969), il Mulino, Bologna 1972.

${ }^{18}$ Cfr.: G. Mosca, Sulla teorica dei governi e sul governo parlamentare (ed. or. 1884); Id., Elementi di scienza politica (ed. or. 1895, 1922), entrambi in G. Mosca, Scritti politici, 2 voll., a cura di G. Sola, Utet, Torino 1982.

19 Cfr. V. Pareto, Trattato di sociologia generale (ed. or. 1916), a cura di G. Busino, Utet, Torino 1988.

${ }^{20}$ Cfr. R. Michels, La sociologia del partito politico nella democrazia moderna (ed. or. 1911), il Mulino, Bologna 1966.

${ }^{21}$ Vedi J.A. Schumpeter, Capitalismo, socialismo, democrazia (ed. or. 1942),

Etas, Milano 1994, p. 257.

${ }^{22}$ Ivi, pp. 239 sgg. 
una volontà popolare. Esistono soltanto tante volontà particolari, definite da interessi specifici. Tali volontà sono solo limitatamente razionali: sulla loro formazione incidono pesantemente la pubblicità e altri strumenti di persuasione ${ }^{23}$. Pertanto, non ha senso neppure postulare una concezione intersoggettiva di bene comune, per il semplice fatto che le persone hanno concezioni diverse e irriducibili di ciò che la vita e la società dovrebbero essere $^{24}$. L'unico compito che la somma delle volontà particolari può assolvere è quello di legittimare le leadership politiche.

La concezione di Schumpeter è discutibile sotto molti punti di vista (per limitarsi a uno solo: se la volontà dei cittadini è artefatta dai messaggi della pubblicità, quali ragioni ci sono per confidare nella capacità degli elettori di scegliere i leader politici più capaci? $\left.{ }^{25}\right)$. Qui, però, merita concentrarsi su un diverso profilo, che ci consente di rileggere il percorso sin qui svolto e di impostare i passi successivi.

Abbiamo visto nel primo capitolo che gli esordi della riflessione sulla democrazia sono segnati dall'esigenza di rispondere alla critica relativa agli esiti potenzialmente distruttivi del governo popolare. A tale critica Aristotele replicava sottolineando che anche un vasto insieme di persone può disporre delle competenze necessarie a esercitare le funzioni di governo, purché tale insieme sia adeguatamente 'qualificato'. In epoca moderna, la questione relativa alla capacità del popolo di governare si lega al problema della legittimazione del potere politico e a quello della fondazione di un ordine sociale giusto. Nel corso dell'Ottocento, la moderna democrazia rappresentativa non è più una forma di governo fra le altre, ma è diventata una sorta di orizzonte della modernità. Autori come Tocqueville e Marx non sono impegnati a difendere o fondare la democrazia ma a svelarne le interne aporie. Questo percorso conosce un ulteriore snodo con l'avvento della democrazia di massa, su cui si esercita la riflessione di Weber. Weber, da un lato reinterpreta la democrazia in senso procedurale, vale a dire non come una forma di governo designata per permettere l'espressione della volontà popolare, ma come un dispositivo tecnico volto a rendere possibile il funzionamento dello Stato. Dall'altro, abbina a questa caratterizzazione una teoria normativa riguardo al 'contenuto' di senso che il leader carismatico deve veicolare.

Adesso, Schumpeter propone una concezione completamente appiattita sulla dimensione descrittiva, in cui la democrazia figura come metodo per conseguire certi risultati, una concezione nella quale l'unico scarto tra il piano dell'essere e quello del dover essere è dato dall'assunzione -

${ }^{23}$ Ivi, pp. 244-252.

${ }^{24}$ Ivi, pp. 239-242.

${ }^{25} \mathrm{Su}$ questo punto, cfr. D. Zolo, Il principato democratico. Per una teoria realistica della democrazia, Feltrinelli, Milano 1992, pp. 171-174. 
difficilmente giustificabile - secondo la quale la competizione democratica rappresenta il migliore accorgimento istituzionale per legittimare i governanti ${ }^{26}$. In tutto questo la democrazia è passata a essere, da una forma di governo da giustificare, a un insieme di accorgimenti istituzionali da studiare, da descrivere e, al limite, da emendare.

Si tratta di un'impostazione che segna l'avvio della riflessione più squisitamente politologica sulla democrazia ${ }^{27}$, che occuperà gran parte del dibattito nella seconda metà del Novecento ${ }^{28}$.

\section{Dopo la prima guerra mondiale}

Dopo la prima guerra mondiale le strutture dello Stato liberale ottocentesco tendono a sgretolarsi. Nei principali paesi dell'Europa Occidentale viene introdotto il suffragio universale mentre cadono gli imperi tedesco e austriaco. In Russia il regime zarista viene abbattuto con la forza. La rivoluzione russa porta con sé la novità del primo Stato in cui si realizzano i principî del marxismo, a partire dalla gestione collettivistica dell'economia e della società. Nel resto d'Europa l'eco della rivoluzione di ottobre si traduce in un'ondata di sommovimenti popolari che generano una reazione di segno opposto che in molti casi sfocia in autoritarismi e dittature.

In Germania, dopo un breve episodio rivoluzionario, viene approvata nel 1919 una nuova Costituzione che presenta rilevanti profili di novità: si tratta della Costituzione di Weimar, alla cui stesura aveva contribuito fra gli altri Max Weber. Gli elementi più significativi sono due: l'architettura istituzionale e la dotazione di diritti. Vediamoli brevemente in successione $^{29}$.

Partendo dal primo profilo, se è indiscutibile che la nuova carta ponga al centro della formazione della volontà politica il Parlamento, essa prevede anche la figura di un Presidente della Repubblica eletto direttamente dal popolo e la presenza di istituti di democrazia diretta come il referendum

${ }^{26}$ Cfr. ivi, pp. 111-114.

${ }^{27}$ Non che profili politologici fossero assenti negli autori classici - a partire da Aristotele.

${ }^{28}$ Cfr. le opere di due autori significativamente influenzati da Schumpeter: Dahl, La democrazia e i suoi critici, cit.; G. Sartori, The Theory of Democracy Revisited, 2 voll., Chatham House Publishers, Chatham 1987. A Schumpeter si richiamano anche i fautori di un approccio 'economico' alla democrazia. Cfr.: A. Downs, Teoria economica della democrazia (ed. or. 1957), il Mulino, Bologna 1989; J.M. Buchanan, G. Tullock, The Calculus of Consent. Logical Foundations of Constitutional Democracy, University of Michigan Press, Ann Arbour 1962.

${ }_{29}$ Per una disamina accurata, cfr. M. Stolleis, Geschichte des öffentlichen Rechts in Deutschland, vol. 3, Staats- und Verwaltungsrechtswissenschaft in Republik und Diktatur 1914-1945, C.H. Beck, München 1999, pp. 90-124. 
e le leggi di iniziativa popolare. Questo assetto misto dà adito a una vasta riflessione intorno ai limiti della democrazia parlamentare, dibattito che era stato sostanzialmente avviato dagli ultimi scritti di Weber ${ }^{30}$ e che vedrà tra i suoi protagonisti Carl Schmitt (1888-1985) e Hans Kelsen (18811973). Schmitt difenderà la tesi dell'opposizione fra parlamentarismo e democrazia, essendo il primo fondato sul pluralismo e la discussione, la seconda sull'omogeneità e la rimozione dell'estraneo ${ }^{31}$; Kelsen, invece, sosterrà la centralità del Parlamento e dei partiti come espressione del pluralismo sociale, pluralismo che attraverso la dialettica parlamentare può trovare sfogo in forme di conflittualità non distruttiva ${ }^{32}$.

Il secondo elemento distintivo della Costituzione di Weimar è dato dal fatto di prevedere una consistente dotazione di diritti sociali, come il diritto all'istruzione e il diritto al lavoro, che si aggiungevano ai tradizionali diritti civili e politici. Questa caratteristica andava a riformare la legislazione sociale prussiana, varata negli anni Ottanta dell'Ottocento, che contemplava schemi assicurativi obbligatori contro la malattia, gli infortuni, la vecchiaia o l'invalidità. In questo modo, l'elargizione di prestazioni positive da parte dello Stato non configurava più una concessione dall'alto ma, per la prima volta, con la parziale eccezione della Costituzione giacobina del 1793, rispondeva a un diritto di cui erano titolari i cittadini ${ }^{33}$.

Il tema dei diritti sociali si collega al tema dell'intervento degli Stati in materia economica. Fino alla fine del XIX secolo la tesi di una separazione netta tra Stato, società ed economia non era messa in discussione. Con l'allargamento del suffragio nella seconda metà dell'Ottocento, lo Stato tende ad assumere un ruolo diretto in materia economica, in primo luogo in Prussia, in concomitanza con i primi esempi di legislazione sociale. Questa tendenza si intensifica con la prima guerra mondiale e soprattutto dopo la grande crisi del 1929. Si inizia a ritenere che l'intervento dello Stato in economia debba diventare strutturale, per scongiurare il ripetersi di crisi economiche. Questa intuizione viene sviluppata in modo originale negli Stati Uniti con il New Deal, il programma di riforme socioeconomiche varato dal presidente Franklin Delano Roosevelt nel 1933 per contrastare

${ }^{30}$ Cfr.: Weber, Parlamento e governo, cit.; Id., Il Presidente del Reich (ed. or. 1919), in Id., Scritti politici, cit., pp. 231-236.

${ }^{31}$ Cfr. C. Schmitt, Dottrina della Costituzione (ed. or. 1928), a cura di A. Caracciolo, Giuffrè, Milano 1984. Su Schmitt, cfr.: G. Preterossi, Carl Schmitt e la tradizione moderna, Laterza, Roma-Bari 1996; S. Pietropaoli, Schmitt, Carocci, Roma 2012.

${ }^{32}$ Cfr. soprattutto i saggi raccolti in H. Kelsen, La democrazia, a cura di M. Barberis, il Mulino, Bologna 1984.

${ }_{33}$ Su questi temi cfr.: J. Alber, Dalla carità allo Stato sociale (ed. or. 1982), il Mulino, Bologna 1986; M. Ferrera, Modelli di solidarietà. Politica e riforme sociali nelle democrazie, il Mulino, Bologna 1993; C. Giorgi, Le istituzioni del welfare, in Meriggi, Tedoldi (a cura di), Storia delle istituzioni politiche, cit., pp. 169-94. 
gli effetti della recessione. Il nucleo di questo approccio si può ritrovare nella concezione economica sviluppata da John Maynard Keynes (18831946), secondo il quale per sostenere la ripresa della domanda è necessario ampliare la spesa pubblica ${ }^{34}$.

Frattanto in Europa, con il fascismo in Italia, il nazismo in Germania e il comunismo in Unione Sovietica, si fa strada una nuova forma di Stato che soppianta lo Stato di diritto: lo Stato totalitario. Gli Stati totalitari sono caratterizzati: $a$ ) da un'ideologia totalizzante, vale a dire un complesso di credenze attinenti non solo alla politica ma alla società stessa e al posto degli individui in essa; $b$ ) da un partito unico che controlla l'apparato statale; $c$ ) dal monopolio dei mezzi di comunicazione; $d$ ) dal ricorso indiscriminato a strumenti di controllo polizieschi; $e$ ) dalla direzione centralizzata dell'economia. In essi non è più pensabile l'esistenza di una legge astratta in funzione di limite ai potenziali abusi del potere e di un corpo legislativo che rappresenta, se non in senso metaforico, la volontà dei cittadini ${ }^{35}$.

\section{Il secondo dopoguerra}

La tragedia della seconda guerra mondiale produsse l'effetto di indurre una grande trasformazione negli assetti politici allo scopo di impedire l'insorgenza di nuove forme di totalitarismo e di assicurare un futuro di pace.

Sul piano interno la novità più rilevante è data dall'avvento dello Stato Costituzionale. Di costituzione nel senso di legge fondamentale, che fissa le regole più importanti dello Stato ed enuncia i diritti degli individui, si inizia a parlare dalla seconda metà del Settecento. Tuttavia, le costituzioni rivoluzionarie e poi quelle ottocentesche possedevano un ruolo più politico che giuridico, avevano la funzione di marcare un importante snodo storico ma non vincolavano il potere legislativo. Per questo si dice abitualmente che le costituzioni ottocentesche erano costituzioni flessibili. Al contrario, le costituzioni del secondo dopoguerra nascono come costituzioni rigide, che possono essere modificate solo attraverso procedure aggravate e tali da prevalere su leggi ordinarie contrastanti - che, come sostenuto da Kelsen, per questo motivo possono essere dichiarate invalide da un apposito Tribunale costituzionale. In altre parole, il dettato costituzionale diventa un limite per il potere legislativo ${ }^{36}$.

34 Cfr. J.M. Keynes, Teoria generale dell'occupazione, dell'interesse e della moneta (ed. or. 1936), a cura di T. Cozzi, Utet, Torino 2006.

35 Sugli Stati totalitari, cfr. A. Meniconi, Le istituzioni politiche del totalitarismo: Italia, Germania, Unione Sovietica, in Meriggi, Tedoldi (a cura di), Storia delle istituzioni politiche, cit., pp. 195-218.

${ }^{36} \mathrm{Cfr}$. i saggi raccolti in H. Kelsen, La giustizia costituzionale, a cura di C. Geraci, Giuffrè, Milano 1981. 
In questo modo, l'idea illuministica e poi ottocentesca della sovranità popolare come fondamento del potere politico, idea che si esprimeva nel primato del legislativo, cede il passo, ancora in accordo con la concezione di Kelsen, al punto di vista secondo il quale il potere dello Stato si fonda sulla sovranità della Costituzione. Ne deriva l'identificazione dello Stato con l'ordinamento giuridico - vale a dire, l'insieme di norme che rinviano alla Costituzione - mentre il Parlamento diventa il luogo in cui vengono dibattute le istanze provenienti dalla società ${ }^{37}$.

Per quanto riguarda il contenuto, le costituzioni del dopoguerra si presentano come costituzioni lunghe, che abbinano dettagliate regole organizzative a un nutrito catalogo di diritti, civili, politici e sociali. Il complesso di tali diritti affida al legislatore un programma che richiede di essere attuato, dal momento che per rendere effettivo il diritto alla salute, all'istruzione $\mathrm{o}$ al lavoro è necessario mettere in piedi un adeguato sistema di norme di settore e un apparato di infrastrutture amministrative ad hoc. La proiezione dello Stato nel campo sociale genera una nuova immagine della cittadinanza, nella quale la dotazione di diritti sociali si affianca a quella di diritti civili e politici, a comporre un nuovo modello di individuo, differente da quello tipizzato dal liberalismo tradizionale ${ }^{38}$.

Sul piano internazionale la novità più rilevante è rappresentata dalla nascita dell'Organizzazione delle Nazioni Unite nel 1945 a San Francisco. La costituzione dell'ONU segna soltanto il momento iniziale di una fase ancora in corso marcata dal tentativo di creare documenti e istituzioni internazionali capaci di garantire la pace, la sicurezza e la cooperazione fra le nazioni: basti pensare, da un lato a documenti come la Dichiarazione Universale dei Diritti dell'Uomo del 1948 e alle numerose convenzioni internazionali - per esempio sulla tortura, sul genocidio, sulle discriminazioni razziali e di genere, sullo statuto dei rifugiati ecc. - dall'altro, a istituzioni come la FAO (Organizzazione delle Nazioni Unite per l'Alimentazione e l'Agricoltura), l'UNICEF (Fondo delle Nazioni Unite per l'Infanzia), l'ILO (Organizzazione Internazionale del Lavoro), l'UNESCO (Organizzazione delle Nazioni Unite per l'Educazione, la Scienza e la Cultura), il WHO (Organizzazione Mondiale della Sanità), la Banca Mondiale, il Fondo Monetario Internazionale, il WTO (l'Organizzazione Mondiale per il Commercio), l'Alto Commissariato per i Diritti Umani, l'Alto Commissariato per i Rifugiati ecc. ${ }^{39}$

${ }_{37}$ Cfr. H. Kelsen, Teoria generale del diritto e dello Stato (ed. or. 1961), a cura di S. Cotta e G. Treves, Edizioni di Comunità, Milano 1963.

${ }^{8}$ Cfr. Th. H. Marshall, Cittadinanza e classe sociale (ed. or. 1963), a cura di P. Maranini, Utet, Torino 1976. Sul tema, cfr. anche D. Zolo (a cura di), La cittadinanza. Appartenenza, identità, diritti, Laterza, Roma-Bari 1994.

39 Per una panoramica sulle istituzioni internazionali, cfr. R. Belloni, M. Moschella, D. Sicurelli (a cura di), Le organizzazioni internazionali. Struttura, funzioni, 
Questi documenti e queste istituzioni presuppongono la formazione di un insieme di principî normativi aventi carattere universale. Inoltre, presuppongono anche la limitazione della sovranità degli Stati aderenti - come è esplicitato dall'art. 11 della Costituzione italiana - nel senso che gli organismi sovranazionali possono svolgere la funzione che è loro affidata solo in quanto gli Stati riconoscano la loro autorità e accettino di limitare di conseguenza il proprio potere. Ma questo trasferimento di sovranità comporta un problema significativo per la teoria democratica, in quanto ne risulta che porzioni della sovranità statale siano state affidate a organismi non dotati di legittimazione democratica. Questo problema si pone con ancora maggiore evidenza nel caso di organizzazioni e documenti regionali, come la Nato, la Convenzione Europea dei Diritti Umani e soprattutto l'Unione Europea, che implicano un trasferimento di poteri più significativo ${ }^{4}$.

Su alcuni aspetti delle democrazie costituzionali ritornerò nel capitolo successivo. Intanto, nelle prossime sezioni introdurrò le coordinate teoriche di alcuni autori della seconda metà del Novecento che risulteranno essenziali per apprezzare i contenuti del dibattito sui presupposti, sulle finalità e sui limiti delle democrazie costituzionali.

\section{Rawls}

La prima prospettiva su cui intendo soffermarmi è quella di John Rawls (1921-2002). Rawls è stato il maggior filosofo della politica di lingua inglese del Novecento. La sua opera più famosa, Una teoria della giustizia, venne pubblicata nel 1971 e rappresenta un tentativo di fondare la legittimità del Welfare State utilizzando l'armamentario teorico del contrattualismo ${ }^{41}$. Questa scelta possiede anche un significato metodologico: nell'opzione contrattualista Rawls vede una possibilità per aggirare il conflitto fra ideologie totalizzanti - come socialismo e utilitarismo definendo le coordinate di una teoria minima di ciò che è giusto.

È importante dire subito che questa riformulazione introduce un sostanziale cambiamento: non si tratta più di legittimare il potere politico, come nei contrattualisti classici, ma di dare un fondamento a principî di

impatto, il Mulino, Bologna 2013. Più in particolare sull'ONU, cfr. A. Polsi, Storia dell'ONU, Laterza, Roma-Bari 2009.

$4^{\circ}$ Cfr. R. Santaniello, Capire l'Unione europea. Politiche, diritto, economia, il Mulino, Bologna 2016.

${ }^{41} \mathrm{Su}$ Rawls, cfr.: S. Freeman, Rawls, Routledge, London 2007; S. Maffettone, Introduzione a Rawls, Laterza, Roma-Bari 2010. Su Rawls e la democrazia, cfr. A. Gutman, Rawls on the Relationship between Liberalism and Democracy, in S. Freeman (ed.), The Cambridge Companion to Rawls, Cambridge, University Press, Cambridge 2003, pp. 168-199. 
giustizia sociale coerenti con la prassi degli Stati contemporanei. Rawls sostiene che la giustizia va concepita come equità, vale a dire come il risultato di un accordo stretto in maniera assolutamente equa per tutti coloro che vi partecipano.

Rawls immagina che un insieme di individui, connotati antropologicamente come decisori razionali, si trovi in una situazione astratta in cui i dettagli riguardo alle rispettive dotazioni individuali - non solo lo status sociale ma anche i particolari relativi alle proprie doti naturali e anche alla propria concezione del bene - sono coperti da un velo di ignoranza ${ }^{42}$. In questa situazione, sostiene Rawls, sarebbe razionale accordarsi su principî di giustizia che tutelassero i meno avvantaggiati, dal momento che non è possibile sapere in anticipo quale posizione ciascuno andrà a occupare in concreto in seno alla società. Questa risposta conduce a due principî di giustizia. Primo: ogni persona ha un uguale diritto al più esteso schema di uguali libertà fondamentali compatibilmente con un simile schema di libertà per gli altri. Secondo: le ineguaglianze sociali ed economiche devono essere combinate in modo da essere (a) ragionevolmente previste a vantaggio di ciascuno; (b) collegate a cariche e posizioni aperte a tutti ${ }^{43}$. Rawls fornisce così un fondamento teorico alle politiche redistributive degli Stati democratici senza abbracciare il principio radicalmente egualitario di un'identica assegnazione di beni per tutti gli individui.

La Teoria della giustizia di Rawls sollevò un amplissimo dibattito. Oltre alle critiche 'neoliberali' di Nozick - di cui parlerò nella prossima sezione - merita qui ricordare le critiche che i pensatori detti 'comunitaristi' - come Alaisdair McIntyre, Michael Sandel, Charles Taylor - rivolsero a Rawls tra la fine degli anni Settanta e i primi anni Ottanta. In estrema sintesi, i comunitaristi criticavano soprattutto un aspetto della proposta rawlsiana: il fatto che la concezione del soggetto politico che Rawls metteva in campo era troppo astratta, quasi ricalcata sul modello di individuo che ci viene consegnato dalla tradizione del liberalismo, mentre le persone in carne e ossa non sono soltanto dei decisori razionali ma spesso agiscono sulla base delle credenze e dei valori della comunità cui appartengono ${ }^{44}$.

In risposta Rawls nel suo libro successivo, Liberalismo politico del 1993, accettava in parte le critiche, riconoscendo che la proposta della sua

${ }^{42}$ Vedi J. Rawls, Una teoria della giustizia (ed. or. 1971, 1999), a cura di S. Maffettone, Feltrinelli, Milano 2008, cap. III.

43 Ivi, p. 76.

${ }_{44}$ Cfr.: A. MacIntyre, Dopo la virtù. Saggio di teoria morale (ed. or. 1981), Feltrinelli, Milano 1988; M.J. Sandel, Il liberalismo e i limiti della giustizia (ed. or. 1982), Feltrinelli, Milano 1994; Ch. Taylor, Radici dell'io. La costruzione dell'identità moderna (ed. or. 1989), Feltrinelli 1993. Cfr. anche i saggi contenuti in A. Ferrara (a cura di), Comunitarismo e liberalismo, Editori Riuniti, Roma 2000. 
teoria della giustizia si inscriveva nella tradizione liberale. Tuttavia, ribadiva anche la validità dei principî di giustizia che aveva difeso nell'opera precedente ${ }^{45}$. In particolare, la strategia seguita da Rawls in Liberalismo politico si incentrava sulla distinzione tra giusto e bene: le società contemporanee - molto di più nel 1993 rispetto all'inizio degli anni Settanta, quando Rawls aveva pubblicato la Teoria della giustizia - vedono la compresenza di una pluralità di concezioni del bene, di cui la concezione liberale costituisce solo un esempio. Nondimeno, sostiene Rawls, tutte le concezioni del bene ragionevoli - vale a dire, che concepiscono la società come un equo sistema di cooperazione, in cui nessuno vive alle spalle degli altri e cerca di imporre le proprie credenze e i propri valori - devono convergere verso un identico insieme di principî di giustizia, che diventa dunque oggetto di un overlapping consensus, un consenso per sovrapposizione. Cambia dunque il modo di giustificare i principî di giustizia, non cambia invece il loro contenuto ${ }^{46}$.

\section{Hayek, Nozick e la tradizione neoliberale}

La teoria della giustizia rawlsiana definisce le coordinate di base di un nuovo progetto politico, delineando una concezione che, da una parte, affonda le sue radici, evidenti nella caratterizzazione antropologica dei soggetti, nella tradizione liberale - da Locke a Mill, da Constant a Tocqueville, per citare solo alcuni nomi - ma che, d'altra parte, accoglie istanze di giustizia sociale di ascendenza socialdemocratica.

Alla tradizione liberale si riallaccia però anche un diverso filone di pensiero, che tende invece a esiti apertamente conservatori. Si tratta dell'orientamento che si può chiamare neoliberale, neoliberista o libertario, per distinguerlo dal liberalismo precedente ${ }^{47}$. Questo orientamento ha il proprio ispiratore più illustre nell'economista e filosofo austriaco Friedrich August von Hayek (1899-1992). Hayek nelle sue opere formula una penetrante critica al socialismo e allo Stato sociale, che del primo considera erede $^{48}$. In particolare, Hayek coglie con precisione l'aporia costitutiva del progetto statuale novecentesco: l'intervento dello Stato nel settore socioeconomico presuppone l'esistenza di un insieme molto vasto di norme pratiche, cui i comportamenti individuali sono subordinati, senza che ci siano motivi sufficienti per ritenere che un «codice etico completo»

45 Cfr. J. Rawls, Liberalismo politico (ed. or. 1993, 1996, 2005), a cura di S. Veca, Einaudi, Torino 2012.

${ }^{46}$ Ivi, lez. IV.

47 Cfr. E. Mack, Libertarianism, Polity Press, Cambridge 2018.

${ }^{48}$ Cfr. la prefazione che Hayek scrisse per l'edizione americana di F.A. Hayek, La via della schiavitù (ed. or. 1944, 1956), a cura di D. Antiseri e R. De Mucci, Rusconi, Milano 1995. 
sia adeguatamente condiviso ${ }^{49}$. Questa critica si precisa in Legge, legislazione e libertà (1973-79), in cui Hayek riconduce l'idea della pianificazione economica alla tendenza costruttivista che ipotizza l'esistenza di un soggetto onnisciente ed esterno alla società. Hayek ritiene che questo presupposto sia insostenibile: non è possibile possedere le conoscenze necessarie per dirigere l'evoluzione del sistema sociale. Stigmatizza di conseguenza la complessificazione e burocratizzazione della macchina statale che comporta una riduzione delle libertà individuali, elogiando, per contro, le capacità autoregolative del sistema economico ${ }^{50}$.

Nel 1974, quando Hayek stava ultimando Legge, legislazione e libertà, viene pubblicato Anarchia, Stato e utopia, l'opera più nota di Robert Nozick (1938-2002), il testo che rappresenta la risposta neoliberale a Una teoria

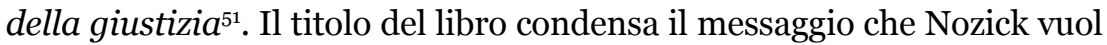
veicolare: da una situazione di anarchia è possibile sollevarsi all'ordine dello Stato assecondando le dinamiche del mercato; il risultato è una forma di Stato differente dallo Stato sociale, lo Stato minimo. Tale modello, tuttavia, è pienamente soddisfacente, tanto da configurare una plausibile concretizzazione dello Stato ideale, cui tende la tradizione utopica.

Il punto di partenza della teoria di Nozick è la nozione lockiana di autoappartenenza: gli individui sono caratterizzati dalla proprietà assoluta della propria persona che fa di loro dei fini in sé e che fonda la loro capacità di acquistare diritti sulle cose nel mondo. Ne segue che l'unica distribuzione legittima dei beni è quella che segue al libero scambio, anche nel caso in cui l'esito conclusivo sia segnato da forti diseguaglianze, e che lo Stato non ha alcun titolo per azionare politiche redistributive della ricchezza. Da qui la conclusione che lo Stato deve limitarsi a predisporre quelle infrastrutture minime che consentono la protezione dei diritti civili e politici, senza attivarsi nel campo sociale. Nella sostanza, quindi, Nozick riprendeva il messaggio politico hayekiano privandolo della cornice epistemologica che ne forniva la motivazione più profonda.

Peraltro, le ricette neoliberali á la Nozick hanno conosciuto un successo politico quasi immediato. A partire dalla seconda metà degli anni Settanta si apre, infatti, una fase di ridimensionamento dello Stato sociale, innescata, fra le altre cose, dalle crisi petrolifere del 1973-74 e del 1979. La congiuntura economica dà adito a una riflessione più vasta che tematizza i limiti dell'intervento dello Stato nel settore socioeconomico, teorizzando

49 Ivi, p. 108.

${ }^{50}$ F.A. von Hayek, Legge, legislazione e libertà. Critica dell'economia pianificata (ed. or. 1973-1979), a cura di A. Petroni e S. Monti Bragadin, il Saggiatore, Milano 1994. Su Hayek, cfr. F. Tedesco, Hayek, Laterza, Roma-Bari 2005.

${ }^{51}$ R. Nozick, Anarchia, Stato e Utopia (ed. or. 1974), presentazione di S. Maffettone, il Saggiatore, Milano 2008. Su Nozick, cfr. R. Bader, Robert Nozick, Bloomsbury, London 2013. 
la contrazione delle istituzioni del Welfare State per 'esonerare' il sistema politico da una domanda di intervento divenuta ormai ingestibile ${ }^{52}$. Tale tendenza si consolida nella prima metà degli anni Ottanta con la presidenza di Ronald Reagan negli Stati Uniti e con il premierato di Margaret Thatcher nel Regno Unito, basati sulla riduzione dell'imposizione fiscale, sulla deregulation del mercato del lavoro e sulla riduzione delle politiche sociali.

\section{La teoria critica e Habermas}

Le trasformazioni prodottesi in seno al sistema politico-economicosociale e il tentativo di comprendere i mutamenti in si riflettono in Germania in un'importante intensificazione del dibattito filosofico-politico. Tra i maggiori protagonisti di questo dibattito si situa certamente Jürgen Habermas (Düsseldorf, 1929).

Habermas si era formato all'interno della Scuola di Francoforte che, sin dagli anni Trenta, aveva avviato un originale percorso di rielaborazione delle categorie del pensiero marxista in rapporto al contesto delle società di massa. In questo modo, autori come Max Horkheimer (1895-1973), Theodor Wiesengrund Adorno (1903-1969) e Herbert Marcuse (18981979) avevano riflettuto criticamente prima sulle trasformazioni della statualità culminate nel nazismo e poi, più in generale, sul rapporto tra politica ed economia e sulle caratteristiche delle società tardo-capitalistiche. A proposito di queste ultime, i Francofortesi avevano messo in luce come in esse il dominio - Herrschaft, categoria chiave della sociologia politica, già elaborata da Weber - non si manifesti apertamente in forme violente ma operi come mercificazione degli ambiti di esistenza sociale, attraverso la manipolazione psicologica e l'industria culturale ${ }^{53}$.

${ }^{2}$ Cfr. il rapporto di Michael Crozier, Samuel Huntington e Jōji Watanuki alla Commissione trilaterale. Cfr. M. Crozier, S.P. Huntington, J. Watanuki, La crisi della democrazia. Rapporto sulla governabilità delle democrazie alla Commissione trilaterale (ed. or. 1975), FrancoAngeli, Milano 1977.

${ }_{53}$ Cfr.: M. Horkheimer, Th.W. Adorno, Dialettica dell'illuminismo (ed. or. 1944), introduzione di C. Galli, Einaudi, Torino 1997; M. Horkheimer, Filosofia e teoria critica, a cura di S. Petrucciani, Einaudi, Torino 2003; Th. W. Adorno, Minima moralia. Meditazioni della vita offesa (ed. or. 1951), a cura di L. Ceppa, Einaudi, Torino 1994; H. Marcuse, Ragione e rivoluzione. Hegel e il sorgere della teoria sociale (ed. or. 1941), introduzione di C. Galli, il Mulino, Bologna 1998; Id., Eros e civiltà (ed. or. 1955), Einaudi, Torino 1967; Id., L’uomo a una dimensione. L'ideologia di una società industriale avanzata (ed. or. 1964), Einaudi, Torino 1967. Sulla scuola di Francoforte, cfr. F. Rush (ed.), The Cambridge Companion to Critical Theory, Cambridge University Pess, Cambridge 2008. Su Adorno, cfr. S. Petrucciani, A lezione da Adorno. Filosofia, società, estetica, Manifestolibri, Roma 2017. Su Marcuse, cfr.: G. Palombella, Ragione e immaginazione. Herbert Marcuse 1928-1955, De Donato, Bari 1982; R. Laudani, Politica come movimento. Il pensiero di Herbert Marcuse, il Mulino, Bologna 2005. 
E proprio l'approfondimento delle modalità nelle quali le logiche di potere si presentano nella società e nella politica nell'età moderna e contemporanea è uno dei tratti caratteristici della riflessione di Habermas. In particolare, in Storia e critica dell'opinione pubblica oggetto dell'indagine habermasiana è la parabola della sfera pubblica ${ }^{54}$ : quest'ultima vede la propria genesi nel XVII secolo, a partire dalle gazzette e dai dispacci mercantili, contemporaneamente all'affermazione dell'economia capitalistica. Si sviluppa nel corso del XVIII secolo, parallelamente alla fioritura del dibattito artistico e culturale, e si costituisce come luogo privilegiato nel quale la borghesia sottopone al vaglio della ragione l'operato dei governanti ${ }^{5}$.

Nel corso del XIX secolo emergono le contraddizioni su cui la funzione politica della sfera pubblica si fonda. I privati cittadini possono esercitare una funzione critica solo nella misura in cui dispongono di tempo e risorse da investire nella propria formazione culturale. Né, d'altra parte, l'allargamento della sfera pubblica ai non proprietari, tra la fine del XIX e l'inizio del XX secolo, con la diffusione, prima dei giornali, successivamente della radio e della televisione, determina la creazione di una nuova opinione critica maggiormente inclusiva. Si verifica, invece una progressiva involuzione, nella quale l'argomentazione razionale viene surrogata dalle logiche manipolative del linguaggio pubblicitario e i partiti politici si trasformano in aziende pubblicitarie ${ }^{56}$.

In definitiva, il pericolo che Habermas addita è quello dell'invasione degli ambiti in cui si esplica l'agire comunicativo - cioè tutte quelle forme di interazione orientate all'intesa, in cui si rende possibile un riconoscimento intersoggettivo non violento - da parte delle logiche tipiche dell'agire strumentale, imposto dalla razionalizzazione e dalla spersonalizzazione dei legami sociali. Il problema principale è rappresentato dal fatto che il sistema socioeconomico, organizzato secondo principî di integrazione razionale, tende a colonizzare il mondo della vita, vale a dire l'ambito nel quale dovrebbe dispiegarsi l'agire comunicativo - come quando la pubblicità, con le sue logiche commerciali, si inserisce nello spazio consacrato alla discussione pubblica dei mezzi di comunicazione di massa ${ }^{57}$.

Nelle sue opere più mature - in particolare in Fatti e norme del 1992 - Habermas ha sostenuto che il diritto può svolgere una funzione di 'cer-

${ }^{54} \mathrm{Su}$ Habermas, cfr.: S. White (ed.), The Cambridge Companion to Habermas, Cambridge University Press, Cambridge 1995; M. Rosenfeld, A. Arato (eds.), $\mathrm{Ha}$ bermas on Law and Democracy, University of California Press, Berkeley 1998; S. Petrucciani, Introduzione ad Habermas, Laterza, Roma-Bari 2000.

${ }_{55}$ Vedi Habermas, Storia e critica dell'opinione pubblica, cit., capp. I-III.

${ }^{56}$ Ivi, capp. V-VI.

${ }^{57}$ Cfr. J. Habermas, Teoria dell'agire comunicativo (ed. or. 1981), 2 voll., a cura di G.E. Rusconi, il Mulino, Bologna 1986. 
niera' tra sistema sociale e mondo della vita, nel senso di poter prefigurare una serie di modalità nelle quali l'assunzione dei ruoli sociali avviene in forme non strumentali. Attraverso le categorie del diritto viene regolata l'interazione tra sistema e mondo della vita: le istanze provenienti dal sistema acquistano, grazie al medium giuridico, una formulazione adeguata agli standard comunicativi ${ }^{58}$. Il diritto, d'altro canto, può esercitare questa funzione perché rappresenta il risultato di un secolare processo di sedimentazione di una precedente vicenda etica e politica. Al tempo stesso, la politica democratica abbisogna per potersi esplicare delle procedure e delle garanzie apprestate dai diritti soggettivi. Pertanto, tra diritti e democrazia si stabilisce un rapporto di cooriginarietà: i diritti, che rendono possibile l'esercizio della sovranità popolare e l'integrazione sociale, si sono affermati attraverso le lotte democratiche; la democrazia stessa, dall'altro lato, è pensabile e attuabile solo a partire da un orizzonte di diritti civili, politici e sociali59.

In questo modo, Habermas riesce a comporre il dissidio tra il polo delle garanzie liberali - i diritti soggettivi - e il polo della sovranità popolare. Questa composizione, però, avviene su un piano procedurale: le norme che regolano la dialettica democratica e ne forniscono le condizioni di validità nel senso che una deliberazione democratica, per ritenersi valida non deve contravvenire a esse - presuppongono - da un lato, e ne sono una specificazione, dall'altro - un più fondamentale principio di universalizzazione - il Principio del discorso o Principio $D-$, di ascendenza kantiana, che stabilisce che «sono valide soltanto le norme di azione che tutti i potenziali interessati potrebbero approvare partecipando a discorsi razionali» ${ }^{60}$.

La teoria habermasiana della democrazia recupera e approfondisce quindi la concezione proceduralista prefigurata da Weber ${ }^{61}$ e difesa in seguito da Schumpeter, senza peraltro rinunciare a un consistente apporto normativo, nella misura in cui promette di enucleare regole per distinguere tra deliberazioni 'valide' e deliberazioni 'invalide'. Allo stesso tempo, sostenendo che tali regole sono il precipitato di una vicenda secolare di lotte emancipative e rappresentano, per così dire, il 'distillato' delle grandi tradizioni etico-politiche, diventa possibile ricostruire un

${ }^{58} \mathrm{Su}$ questo punto, cfr. le osservazioni critiche di Virginio Marzocchi in V. Marzocchi, Per un'etica pubblica. Giustificare la democrazia, Liguori, Napoli 2000, capp. 5-6.

59 Vedi J. Habermas, Fatti e norme. Contributi a una teoria discorsiva del diritto e della democrazia (ed. or. 1992), a cura di L. Ceppa, Guerini e Associati, Milano 1996, p. 128.

${ }^{60}$ Ivi, p. 131.

${ }^{61}$ Sul nesso tra la teoria democratica di Habermas e la sociologia politica di Weber, cfr. J.P. McCormick, Weber, Habermas and Transformations of the European State: Constitutional, Social, and Supranational Democracy, Cambridge University Press, Cambridge 2007. 
orizzonte di norme procedurali atto a costringere la mutevole realtà delle decisioni democratiche.

\section{Foucault}

Il modello dello Stato sociale, quale emergeva dall'esperienza del secondo dopoguerra, riceve però anche le contestazioni dei settori più radicali della Sinistra, legati all'eredità del marxismo. In Francia, per esempio, questo atteggiamento è evidente sin dagli anni Sessanta negli scritti di Louis Althusser (1918-1981). In estrema sintesi, si può dire che le critiche di Althusser mettono in risalto le trasformazioni cui vanno incontro le relazioni di dominio all'interno degli Stati contemporanei e il ruolo svolto dagli 'apparati ideologici di Stato', cioè quelle strutture - come scuola, chiesa e famiglia - che riproducono le relazioni di potere esistenti ${ }^{62}$.

Sempre in Francia, nel decennio successivo Michel Foucault (19261984), già compagno di studi di Althusser, elabora, con il concetto di 'governamentalità', un modo nuovo di problematizzare l'azione dello Stato ${ }^{63}$. Foucault, nell'ambito di una ricchissima ricognizione critica intorno alla genesi di quella costellazione di concetti, politiche e pratiche che fa da sfondo all'età presente, si sofferma a indagare le tecniche e le tattiche con cui si esprime la dominazione. Questa opzione comporta un ribaltamento di prospettiva: non sono tanto le grandi istituzioni a testimoniare l'esercizio del potere, né sono i trattati dei filosofi o dei giuristi a esplicitarne le modalità. Il potere si perpetua soprattutto in una miriade di pratiche e di tecniche minute che governano il funzionamento di istituti come manicomi, fabbriche, collegi, ospedali, prigioni e che sono codificate in manuali e regolamenti che ne organizzano l'attività ${ }^{64}$.

Sul finire degli anni Settanta, nei corsi tenuti al Collège de France, Foucault si dedica a un'esplorazione del potere che lo metta in relazione con i processi di costruzione di soggettività. Il soggetto non è pensabile se non in connessione con i rapporti di potere entro i quali viene costruito il suo profilo e che conferiscono a esso visibilità sociale e politica. Ne deriva un rinnovamento completo del lessico politico che svela la finzione che si cela dietro nozioni come sovranità e consenso ${ }^{65}$.

${ }^{62}$ Di Althusser, cfr. soprattutto L. Althusser, Per Marx (ed. or. 1965), nota introduttiva di C. Luporini, Editori Riuniti, Roma 1974. Cfr. anche i saggi raccolti in Id., Lo Stato e i suoi apparati, a cura di R. Finelli, Editori Riuniti, Roma 1997.

${ }_{63}^{6}$ Su Foucault, cfr. M. Iofrida, D. Melegari, Foucault, Carocci, Roma 2017.

${ }^{64}$ Cfr.: M. Foucault, Storia della follia nell'età classica (ed. or. 1961), Bur, Milano 2011; Id., Nascita della clinica (ed. or. 1963), Einaudi, Torino 1965; Id., Sorvegliare e punire (ed. or. 1975), Einaudi, Torino 1976.

${ }^{65}$ Cfr. M. Foucault, «Bisogna difendere la società» (ed. or. 1997), a cura di M. Bertani e A. Fontana, Feltrinelli, Milano 1998. 
L'ubiquità del potere comporta da un lato l'impossibilità di una progettualità politica che faccia astrazione dai contesti particolari. Dall'altro, induce a pensare l'emergere stesso dello Stato sociale moderno nei termini di un complesso di strategie atte a 'governare' il corpo molteplice della 'popolazione', in termini 'governamentali', appunto ${ }^{66}$. Ma questo punto di vista è inconciliabile con ogni tentativo di fondare la legittimità dello Stato democratico. La ricerca di Foucault suggerisce piuttosto la possibilità di attestarsi su una serie di linee di resistenza, intese come posizioni che limitano l'esercizio del potere.

Riassumendo, abbiamo visto come nel Novecento il modello liberaldemocratico venga sottoposto a una significativa torsione. Il problema di sfondo è il fatto del pluralismo, che impedisce di perpetuare l'illusione moderna della possibilità della composizione dei diversi interessi e delle diverse visioni del mondo nel segno di una volontà generale preordinata al bene comune. Di fonte a questa impasse si aprono due strade: da una parte, un numero significativo di autori - da Weber a Schumpeter - enfatizza la dimensione procedurale del modello democratico, sostenendo che le differenze devono essere composte nel rispetto di un insieme di regole tecniche; dall'altra, autori ascrivibili alla tradizione liberale - come Rawls - tendono a istituire un assetto dualistico, distinguendo tra il piano universale della giustizia e quello particolare delle diverse concezioni del bene. Queste due strade si trovano, per così dire, sintetizzate nella proposta di Habermas, che fornisce una ricostruzione 'discorsiva' delle regole democratiche, connettendo sovranità popolare e diritti fondamentali e sottolineando come l'una e gli altri siano il precipitato di una originaria sostanza etica.

Tuttavia, questa via di uscita presenta alcune difficoltà. Per un verso, come ha fatto notare in particolare Hayek, uno Stato democratico che si ponga finalità di intervento sociale presta il fianco alla critica secondo la quale un programma di intervento di questo tipo dovrebbe godere di un consenso trasversale, che in una condizione di elevato pluralismo non è possibile presumere. Per un altro, come l'approccio foucaultiano dimostra, qualsiasi strategia di welfare presuppone l'implementazione di tecniche e pratiche governamentali, cui si accompagna un inevitabile portato di esclusione e di disciplinamento ${ }^{67}$.

${ }^{66}$ Cfr. M. Foucault, Nascita della biopolitica (ed. or. 2004), a cura di M. Senellart, Feltrinelli, Milano 2005.

${ }_{7}^{67}$ Questa osservazione non implica l'esistenza di una convergenza tra neoliberalismo e approccio foucaultiano, convergenza che pure è stata tematizzata in alcuni studi recenti: cfr. D. Zamora, M.C. Behrent (eds.), Foucault and Neoliberalism, Polity Press, Cambridge 2015. Sul tema degli esiti disciplinanti delle politiche di welfare, cfr. anche I.M. Young, Le politiche della differenza, Feltrinelli, Milano 1996. 
La conclusione è quindi che il tentativo di rimodulare lo schema della teoria democratica adattandolo alle condizioni presenti si regge su un equilibrio instabile. Nel momento in cui, al termine del XX secolo, il pluralismo sociale sottostante si farà ancora più radicale, i presupposti di quel tentativo salteranno, lasciando via libera alla possibilità di definire un nuovo assetto. 



\section{Capitolo quinto \\ La democrazia costituzionale}

\section{I limiti della democrazia}

All'indomani della rivoluzione francese la legge diventò la fonte del diritto per eccellenza, manifestazione della sovranità popolare: la volontà del popolo sovrano trova espressione nella legge generale e astratta, approvata dai parlamenti in cui siedono i suoi rappresentanti. Questa centralità si conferma nell'Ottocento, collegandosi al mito della Nazione. L’idea dell'illimitatezza della sovranità popolare si rivela però pericolosa, nel momento in cui la Nazione, romanticamente intesa, diventa comunità di destino, Popolo unito da vincoli prepolitici. Infatti, se si ammette che il soggetto depositario della sovranità è una comunità unita da vincoli anteriori alla politica e se, d'altra parte, tale sovranità non può essere limitata da meccanismi interni al quadro istituzionale, si ottiene che l'intero apparato statale viene subordinato al perseguimento di fini anteriori a quelli che emergono nel discorso pubblico. La conseguenza è l'ascesa dei totalitarismi che conducono al sovvertimento dei principî dello Stato di diritto'.

Per evitare la deriva totalitaria gli Stati del dopoguerra vengono riedificati come democrazie costituzionali, dotate di una Costituzione rigida, che non può essere modificata dalla legge ordinaria. Per esempio, nel caso della Costituzione italiana, la Costituzione stessa prevede limiti alla propria revisione, sia per quanto riguarda il contenuto della stessa - la forma repubblicana e, si ritiene generalmente, i principî supremi dell'or-

${ }^{1}$ Su nazioni e nazionalismo, cfr.: B. Anderson, Comunità immaginate. Origini e fortuna dei nazionalismi (ed. or. 1983, 1991), prefazione di M. D'Eramo, Laterza, Roma-Bari 2018; E. Gellner, Nazioni e nazionalismo (ed. or. 1983), Editori Riuniti, Roma 1992.

Leonardo Marchettoni, Breve storia della democrazia: da Atene al populismo, ISBN 978-88-6453760-3 (print) ISBN 978-88-6453-761-o (online PDF) ISBN 978-88-6453-762-7 (online EPUB),

(C) 2018 Firenze University Press 
dinamento costituzionale non possono essere oggetto di revisione - sia per quanto riguarda le forme - le leggi di revisione costituzionale devono essere approvate con un procedimento aggravato, da maggioranze più estese di quelle abituali ${ }^{2}$.

È importante notare che il trapasso dallo Stato di diritto di matrice ottocentesca allo Stato costituzionale si accompagna all'archiviazione dell'idea di sovranità popolare illimitata come fondamento del potere politico. In altre parole, l'azione dello Stato non necessita più di un ancoramento nella nozione metafisica di volontà generale come espressione della sovranità del popolo per legittimarsi, dal momento che la garanzia di legittimità politica e di validità giuridica degli atti della macchina statale va rintracciata nella loro conformità alla Costituzione, quale vertice dell'ordinamento, che diventa esso stesso depositario della sovranità. Ciò significa che all'interno del quadro degli Stati costituzionali, il corpo politico può esercitare il potere legislativo soltanto nel rispetto dei contenuti e dei valori inscritti nella Costituzione.

Questo trapasso, del resto, è conforme alle trasformazioni avvenute nel pensiero politico tra Ottocento e Novecento, quando le riflessioni di autori come Tocqueville e Marx, prima, Weber e Schumpeter, poi, avevano contribuito a svelare il carattere chimerico della nozione di volontà popolare che era stata assunta come architrave del pensiero politico moderno. Il modello a due livelli dello Stato costituzionale è invece perfettamente in linea con la separazione rawlsiana tra i principî di giustizia e le concezioni sostantive della vita buona, che replica la dicotomia tra principî costituzionali e scelte politiche dei singoli governi. Ma anche la teoria discorsiva della democrazia di Habermas sembra riconducibile a questo paradigma, nella misura in cui assume che le regole che presiedono allo svolgimento del dibattito democratico mirino ad assicurare il pieno coinvolgimento di tutti gli interessati e quindi, possano essere ritenute oggettivamente giuste rispetto alle concezioni soggettive del bene ${ }^{3}$.

Chiaramente, questo modello richiede un controllo di tipo giurisdizionale sul contenuto della legge: questo controllo - che affianca il controllo svolto dal giudice amministrativo sugli atti del potere esecutivo, che sostanzia il principio di legalità - viene riservato a un apposito tribunale costituzionale, come la Corte Costituzionale italiana. Le costituzioni rigide definiscono un assetto nel quale la maggioranza parlamentare incontra dei limiti nell'esercizio del proprio potere, anche limiti di principio che

${ }^{2}$ Limiti molto più penetranti alla revisione costituzionale sono previsti, per esempio, dal Grundgesetz tedesco, secondo il quale, non solo i diritti fondamentali ma anche le norme attinenti all'articolazione federale non sono rivedibili.

3 Sull'assetto dualista sotteso agli approcci di Rawls e Habermas, cfr. G. Palombella, È possibile una legalità globale? Il Rule of law e la governance del mondo, il Mulino, Bologna 2012, pp. 49-55. 
sono indipendenti dal valore numerico della maggioranza - persino l'unanimità dei parlamentari non potrebbe apportare certe modifiche alla Costituzione, per esempio, modifiche che introducessero discriminazioni immotivate o limitassero i diritti fondamentali. Queste norme definiscono, per così dire, le regole del gioco democratico, la cornice entro la quale esso si deve svolgere.

Quest'ultimo punto induce a riflettere brevemente sul nesso ambivalente che lega diritti e democrazia. Chiaramente, i diritti si pongono in primo luogo come argini all'abuso del potere. In quanto tali devono essere collocati in una posizione sovraordinata rispetto al sistema democratico e devono essere connotati da un carattere 'astratto'. Al tempo stesso, costituiscono un prodotto della politica e quindi, entro un sistema democratico, di una comunità specifica. In questo senso, possiedono inevitabilmente un carattere più 'concreto' e particolaristico, eventualmente legato alla difesa di interessi di parte. Anche per questo motivo, si distingue spesso tra diritti umani - astratti, sovraordinati all'ordinamento - e diritti fondamentali. Evidentemente, questi due profili sono in tensione fra loro e non è semplice capire in che modo sia possibile, volta per volta, ricucire il loro dissidio ${ }^{4}$.

\section{I diritti fondamentali}

Le costituzioni moderne contengono - generalmente nella prima parte - un catalogo più o meno ampio di diritti fondamentali. L'inserimento di questo catalogo comporta un'apertura dell'ordinamento statuale al riconoscimento di posizioni soggettive che travalicano l'ordinamento stesso, corrispondendo a previsioni normative formulate da documenti sovranazionali o internazionali - la Dichiarazione Universale dei Diritti dell'Uomo, la Convenzione Europea dei Diritti dell'Uomo, ora la Carta dei Diritti Fondamentali dell'Unione Europea (Carta di Nizza). Emblematico è, a questo riguardo, l'art. 2 della Costituzione italiana che recita: «La Repubblica riconosce e garantisce i diritti inviolabili dell'uomo [...]» (corsivo mio). Il fatto che si riconoscano e garantiscano norme che non hanno origine nell'ordinamento comporta che i relativi articoli siano immodificabili5.

I diritti fondamentali, d'altra parte, sono ripartiti in diverse categorie $^{6}$. La categoria più antica è quella dei diritti civili. I diritti civili - come

${ }_{4}$ Per un'approfondita elaborazione della distinzione tra diritti umani e diritti fondamentali, cfr. G. Palombella, Dopo la certezza. Il diritto in equilibrio tra giustizia e democrazia, Dedalo, Bari 2006, cap. 1.

5 Cfr. G. Zagrebelsky, Il diritto mite. Legge diritti giustizia, Einaudi, Torino 1992, cap. 3 .

${ }^{6}$ Sull'evoluzione del sistema dei diritti, cfr.: A. Facchi, Breve storia dei diritti umani. Dai diritti dell'uomo ai diritti delle donne, il Mulino, Bologna 2013; E. Pariotti, I diritti umani. Concetto, teoria, evoluzione, Cedam, Milano 2013. 
il diritto alla vita, il diritto alla libertà, il diritto di proprietà, la libertà religiosa, la libertà di opinione - riguardano il rapporto fra l'individuo e lo Stato, assegnando al primo una sfera di libertà che non può essere invasa dal secondo se non con le modalità previste dalla legge. Le loro prime formulazioni datano agli albori della tradizione liberale: vengono teorizzati da Locke come limiti al potere del sovrano nel Secondo trattato sul governo e contestualmente vengono sanciti nel Bill of Rights.

La categoria successiva è quella dei diritti politici, che riguardano la libertà positiva di partecipare alla gestione dello Stato. I diritti politici che non comprendono soltanto il diritto di voto, attivo e passivo, ma anche i diritti strumentali rispetto alla partecipazione politica, come il diritto di riunione e quello di associazione - vengono solennemente proclamati nelle dichiarazioni rivoluzionarie: la Dichiarazione dei Diritti dell'Uomo e del Cittadino stabilisce che ogni cittadino ha diritto di contribuire alla formazione della legge. Il secolo che è contraddistinto da lotte per la loro espansione è l'Ottocento, al termine del quale si creeranno le condizioni per l'estensione universale del suffragio.

Un discorso a parte meritano i diritti sociali. I diritti sociali sanciscono che gli individui hanno titolo a prestazioni positive da parte dello Stato come il diritto all'istruzione, il diritto alla salute, il diritto al lavoro. Dopo una fuggevole anticipazione con la Costituzione francese del 1793, i diritti sociali si sono diffusi nel Novecento, a partire dalla Costituzione di Weimar, fino alle dichiarazioni internazionali e alle costituzioni democratiche del secondo dopoguerra7.

Dal momento che richiedono una prestazione attiva da parte dello Stato - il quale dovrà evidentemente predisporre le strutture idonee a impartire, per esempio, un'istruzione gratuita - i diritti sociali sembrano differenziarsi dai diritti civili e politici. Conseguentemente, si è avvertito il bisogno di giustificarne l'inclusione nel catalogo dei diritti fondamentali. A questo proposito, si possono seguire due strategie: da un lato, alcuni autori, come Habermas, ritengono che la previsione dei diritti sociali possa essere giustificata strumentalmente, perché i diritti sociali forniscono quelle precondizioni indispensabili per il godimento dei diritti civili e politici: non è possibile esercitare realmente la propria libertà di opinione o partecipare consapevolmente alle scelte politiche se non si ha accesso ad adeguate prestazioni socioeconomiche (in termini di istruzione, salute, lavoro ecc.) ${ }^{8}$.

Diversamente, altri autori come Luigi Ferrajoli ritengono che i diritti sociali rappresentino ormai un fine in sé, nel senso di costituire un

7 Per un approfondimento, storico e concettuale, sui diritti sociali, cfr. Th. Casadei, I diritti sociali. Un percorso filosofico-giuridico, Firenze University Press, Firenze 2012.

${ }^{8}$ Vedi Habermas, Fatti e norme, cit., p. 149. 
obiettivo autonomo che lo Stato si propone di conseguire, al di là della ricaduta positiva che il godimento dei diritti sociali può comportare sugli altri diritti. Secondo Ferrajoli il riconoscimento di diritti sociali rappresenta, per così dire, la conseguenza di una trasformazione antropologica profonda: nel mondo contemporaneo cose precedentemente impossibili sono diventate socialmente possibili - per esempio, la cura di molte malattie - mentre altre che prima erano individualmente possibili sono diventate impossibili - come la sopravvivenza al di fuori dei legami con la civiltà. I diritti sociali sarebbero pertanto il contrassegno della rinnovata socialità degli esseri umani ${ }^{9}$.

Diritti civili, politici e sociali sono le categorie di diritti più antichi. A partire dal secondo dopoguerra sono state immaginate nuove categorie di diritti: i diritti di terza generazione - la prima generazione dei diritti raccoglie i diritti civili e politici, mentre la seconda generazione comprende i diritti sociali -, formata dai diritti collettivi, alla pace, all'autodeterminazione, a un ambiente non inquinato ecc., e persino quelli di quarta generazione, come i diritti all'integrità genetica e all'autonomia cognitiva. Questi esempi testimoniano che lo strumento del diritto soggettivo possiede una perdurante vitalità che lo rende atto a fronteggiare le sfide poste dal mondo contemporaneo ${ }^{10}$.

\section{Costituzioni e pluralismo}

Gli Stati totalitari sposavano un'ideologia monista, nel senso di fondarsi su un insieme di principî e convinzioni fra di loro coesi anteriori allo Stato - concernenti, per esempio, il popolo, il ruolo del partito, una certa visione della storia - e tali da delineare un'identità fortemente esclusiva. All'indomani della seconda guerra mondiale i nuovi Stati si dettero costituzioni che scongiurassero il ripetersi di quelle vicende. Per questo motivo, le costituzioni democratiche europee del secondo dopoguerra sono improntate a un marcato pluralismo. Questa scelta si basa sull'assunto che il pluralismo - religioso, sociale, ideologico - interno allo Stato deve essere rispettato e tutelato. Per esempio, l'art. 3 della Costituzione italiana recita: «Tutti i cittadini hanno pari dignità sociale e sono uguali davanti alla legge senza distinzione di sesso, di razza, di lingua, di religione, di opinioni politiche, di condizioni personali e sociali». Questa scelta sembra

9 Vedi L. Ferrajoli, La democrazia attraverso i diritti, Laterza, Roma-Bari 2013, pp. 214-216. Sul rapporto tra Stato sociale e democrazia, cfr. anche G. Preterossi, Ciò che resta della democrazia, Laterza, Roma-Bari 2015, pp. 16-38. Del carattere 'intersoggettivo' e 'partecipativo' dei diritti sociali parla anche Casadei: vedi Casadei, I diritti sociali, cit., pp. 41-42.

${ }^{10}$ Sulle 'generazioni' dei diritti, cfr. N. Bobbio, L'età dei diritti, Einaudi, Torino 1990, pp. XIII-XV. 
sposare l'idea secondo la quale le visioni particolari del bene sono subordinate ad alcuni principî universali di giustizia che servono a regolarne i reciproci rapporti ${ }^{11}$.

La Costituzione - mi riferisco in questo caso alla Costituzione italiana ma analogo discorso può essere svolto per molte sue omologhe - sposa un'attitudine pluralistica innanzitutto in materia religiosa. Mentre i Patti Lateranensi del 1929, nel contesto dello Statuto Albertino, facevano del Cattolicesimo la religione di Stato, uno dei principî supremi dell'ordinamento costituzionale è il principio di laicità, che comporta, secondo la giurisprudenza della Corte Costituzionale, l'equidistanza dello Stato dalle singole confessioni religiose ${ }^{12}$. Ma anche con riferimento agli indirizzi politico-ideologici potrebbe essere svolta una riflessione simile, sulla base della constatazione che la Costituzione stessa è frutto di un compromesso fra diversi orientamenti politico-ideologici ${ }^{13}$.

Questo assetto ha sostanzialmente retto fino agli anni Novanta del secolo scorso, quando, di fronte all'aumento dei flussi migratori internazionali, lo schema dualistico basato sulla dicotomia principî di giustizia/ concezioni particolari del bene si è mostrato incapace di reggere l'urto delle forme di pluralismo radicale ${ }^{14}$ con le quali gli ordinamenti europei si trovavano sempre più spesso a doversi confrontare. Questo perché il principio di neutralità dello Stato liberale, inteso come equidistanza dalle diverse identità culturali o religiose si è rivelato impraticabile. Il problema è che certe decisioni fondamentali su cui è basata la convivenza - decisioni relative per esempio alla lingua da adottare nella società, alla scelta delle festività pubbliche, al modo stesso di organizzare la vita comune - non possono essere neutrali rispetto alle diverse appartenenze. Dall'altro lato, i fondamenti stessi dello Stato costituzionale vengono posti in dubbio rispetto alla loro origine occidentale - vedi il dibattito ricorrente intorno al problema dell'universalità dei diritti umani ${ }^{15}$.

${ }^{11}$ Cfr. Zagrebelsky, Il diritto mite, cit. Per una problematizzazione della posizione di Zagrebelsky, cfr. G. Palombella, Costituzione e sovranità. Il senso della democrazia costituzionale, Dedalo, Bari 1997, cap. 9.

${ }^{12}$ Vedi le sentenze 203/1989 e 503/2000. Sul tema, cfr. P. Consorti, Diritto e religione, Laterza, Roma-Bari 2014, pp. 29-46.

${ }_{13}$ Cfr. Petrucciani, Democrazia, cit., pp. 155-162.

${ }_{14}$ Alessandro Ferrara parla a questo proposito di «iperpluralismo». Vedi A. Ferrara, The Democratic Horizon, Cambridge University Press, New York 2014, cap. 4. Affine a esso è il concetto di «super-diversità», introdotto da Steven Vertovec, che evidenzia che le differenti appartenenze presenti all'interno della società si intersecano, generando sempre nuove combinazioni che mettono in crisi i canali tradizionali della rappresentanza. Vedi S. Vertovec, Super-diversity and its implications, «Ethnic and Racial Studies», XXX (2007), 6, pp. 1024-1054.

${ }_{15}$ Sul tema mi sia consentito rinviare a L. Marchettoni, I diritti umani tra universalismo e particolarismo, Giappichelli, Torino 2012. 
A questo stato di cose ha cercato di rispondere il paradigma multiculturalista, basato sulla compresenza e sul riconoscimento delle diverse culture, che si è affermato sul finire del secolo scorso, con autori come il già citato Charles Taylor e Will Kymlicka. Questa via d'uscita, tuttavia, presuppone un'indebita ipostatizzazione del fattore «cultura»: le culture cui si riferiscono i multiculturalisti sembrano 'blocchi statici', a differenza di quanto sostengono gli antropologi, che sottolineano soprattutto l'aspetto transeunte e artificiale dei costrutti culturali ${ }^{16}$. Inoltre, il multiculturalismo tratta la cultura più come un mezzo che come un fine in sé, dal momento che attribuisce importanza all'appartenenza culturale come ingrediente di un'ideale bildung ${ }^{17}$.

Questa situazione di crisi del modello dualistico che faceva da sfondo al pluralismo costituzionale si è inasprita negli ultimi dieci anni. Il risultato è stata una diffusa chiusura verso le istanze pluraliste. Questo atteggiamento è epitomizzato da un modello costituzionale alternativo, basato sulla riaffermazione dell'identità - culturale, linguistica, religiosa - del popolo. Per esempio, la Legge fondamentale ungherese del $2012 \mathrm{si}$ apre con un marcato riferimento all'identità storica, culturale e religiosa della Nazione ungherese:

WE, THE MEMBERS OF THE HUNGARIAN NATION [...]

We are proud that our king Saint Stephen built the Hungarian State on solid ground and made our country a part of Christian Europe one thousand years ago. [...]

We recognise the role of Christianity in preserving nationhood. We value the various religious traditions of our country. [...]

We commit ourselves to promoting and safeguarding our heritage, our unique language, Hungarian culture and the languages and cultures of national minorities living in Hungary. [...] (corsivi miei) ${ }^{18}$

È interessante notare che la svolta monistica esemplificata dalla Legge fondamentale ungherese si muove in una direzione opposta rispetto alle

${ }^{16}$ Un testo classico, a questo proposito, è J. Clifford, I frutti puri impazziscono. Etnografia, letteratura e arte nel secolo XX (ed. or. 1988), Boringhieri, Torino 1999.

${ }_{17}$ In generale, su questi temi, vedi Marchettoni, Relativismo e differenza culturale, cit., pp. 171-187. Su multiculturalismo e diritti, vedi A. Facchi, I diritti nell'Europa multiculturale. Pluralismo normativo e immigrazione, Laterza, Roma-Bari 2001.

${ }_{18}$ Cito dal testo inglese ufficiale della Legge fondamentale ungherese, disponibile sul sito del Governo ungherese all'indirizzo <http://www.kormany.hu/ download/a/68/1100o/The_Fundamental_Law_of_Hungary_01072016.pdf > (10/2018). Sulla Legge fondamentale ungherese, vedi J.-W. Müller, Cos'è il populismo? (ed. or. 2016), Università Bocconi Editore, Milano 2017, pp. 83-85. Müller parla di «Costituzione faziosa», che fissa alcune preferenze politiche specifiche, che in precedenza sarebbero stato oggetto di dibattito politico, escludendo di conseguenza i partiti di opposizione. 
critiche neoliberali. Come si è visto nel capitolo precedente, autori come Hayek e Nozick avevano criticato l'assunto redistributivo dello Stato sociale sostenendo che il potere politico deve mirare unicamente alla tutela dei diritti fondamentali della tradizione liberale e del Rule of Law. Il ripiegamento identitario, invece, si incardina su un progetto politico più ricco, nutrito del richiamo a valori morali e religiosi, più che a un modello economico e sociale ${ }^{19}$.

Per comprendere meglio le coordinate di questo progetto può essere utile riferirsi ad alcune considerazioni sviluppate da Danilo Zolo. Sostiene Zolo che la funzione principale dei sistemi politici consiste nella riduzione delle paura degli individui attraverso la selezione della complessità dell'ambiente sociale in cui essi si trovano a vivere ${ }^{20}$. Tale funzione viene esercitata tanto più efficacemente quanto più viene implementato il codice incluso/escluso, quanto più viene reso «organico ed esclusivo il vincolo interno della fedeltà politica ${ }^{21}$. Alla luce di queste considerazioni, si può sostenere che la svolta monista e identitaria sia un modo per reagire all'esplosione di complessità verificatesi nel continente europeo nell'ultimo decennio del XX secolo e nei primi anni del XXI, soprattutto nei paesi dell'Est Europa.

L'esempio delle riforme costituzionali ungheresi porta a riflettere sui limiti della rigidità costituzionale. Come è noto, la nuova Legge fondamentale ungherese è stata approvata da una forza di governo che disponeva della maggioranza necessaria - i due terzi dell'Assemblea nazionale - per la revisione. Quindi la rigidità del dettato costituzionale precedente non è valsa a impedire riforme unilaterali. Da questo punto di vista, il quadro non è molto dissimile da quello realizzatosi con l'ascesa al potere dei totalitarismi. Tanto più che anche in Ungheria, come pure in Polonia, la svolta in senso identitario e autoritario ha coinciso con la riscoperta delle specificità culturali, religiose, storiche dei due popoli e con l'indebolimento del principio fondamentale della separazione dei poteri, con il potere esecutivo, appoggiato da una larghissima maggioranza in seno all'assemblea legislativa, che tenta di minare l'indipendenza del potere giudiziario.

La conseguenza è che il meccanismo di revisione costituzionale viene retrocesso al livello di uno strumento di politica governativa, con ciò sostanzialmente obliterando la distinzione tra il piano della legge costituzionale e quello della legge ordinaria ${ }^{22}$. Si perde, così, l'assetto

19 Un simile discorso vale non solo per l'Ungheria ma per molte altre 'democrazie illiberali', come Polonia, Russia, Turchia.

${ }^{20}$ Vedi Zolo, Il principato democratico, cit., pp. 74-76.

${ }^{21}$ Ivi, p. 77.

${ }^{22}$ Le vicende del settimo emendamento della già citata Legge fondamentale ungherese, con l'inserimento di previsioni che nulla hanno a che fare con il carattere 
dualistico che consentiva di 'schermare' il governo democratico dagli effetti potenzialmente perversi della 'tirannide della maggioranza'.

\section{Parlamenti e partiti}

Le recenti vicende del costituzionalismo inducono a riflettere in termini più generali sul governo parlamentare. Come si è detto, i parlamenti nascono nel medioevo come istituzionalizzazione dei consigli di corte. In origine, erano composti da un ristretto insieme di nobili e di alti prelati, della cui opinione il re doveva tenere conto, soprattutto per quanto riguarda la politica estera e fiscale. In Inghilterra il Parlamento assunse il ruolo di antagonista del re, ruolo che avrebbe definitivamente conquistato dopo la Glorious Revolution. Nel resto d'Europa e negli Stati Uniti le assemblee legislative si affermano nel corso dell'Ottocento diventando una reale controparte del potere esecutivo, mano a mano che l'estensione del suffragio incrementava il numero degli elettori. L'ascesa del potere dei parlamenti fra Seicento e Ottocento coincide con l'acquisizione, da parte di questi organi, di un ruolo di produzione del diritto e di legittimazione del potere esecutivo tramite l'istituto della fiducia parlamentare.

Quest'ultima trasformazione modifica anche il ruolo dei partiti. In origine, i partiti si presentano come orientamenti interni alle assemblee - Tories e Whigs nel caso del Parlamento inglese, Destra e Sinistra per l'Assemblea nazionale della Francia rivoluzionaria - con una debole organizzazione interna. Quasi per tutto l'Ottocento, per esempio nell'Italia post-unitaria, i partiti operano come comitati elettorali: piccoli gruppi di persone che si accordano, in vista degli appuntamenti elettorali, per favorire, sulla base di un programma generico, l'elezione di un notabile, il quale, in cambio, si impegna a promuovere gli interessi dei suoi sostenitori. All'indomani delle elezioni, il partito-comitato si scioglie mentre l'eletto confluirà in uno schieramento parlamentare organizzato sulla base di vincoli orizzontali. L'assenza di una reale organizzazione verticale rende possibili repentini cambi di schieramento, il cosiddetto trasformismo ${ }^{23}$.

La situazione muta sul finire del secolo con la diffusione dei primi partiti di massa - il primo, come detto nel capitolo precedente, è il Par-

costituzionale del documento, come la messa al bando dei senza fissa dimora, sono particolarmente istruttive a questo riguardo. Sul tema, cfr. il report di Amnesty International, disponibile all'indirizzo <https://d21zrvtkxtd6ae.cloudfront.net/ public/uploads/2018/o6/20172321/Hungary-Briefing_20-June-2018_FINAL.pdf> (10/2018).

${ }^{23}$ Sui partiti di notabili, cfr.: Manin, Principî del governo rappresentativo, cit., pp. 225-29; della Porta, I partiti politici, cit., pp. 24-28. 
tito Socialdemocratico tedesco nel 1875 . La ragione profonda che motiva la nascita dei partiti di massa è l'estensione del suffragio: l'ammissione dei proletari al voto genera un potenziale bacino di consensi che i partiti tradizionali non erano in grado di sfruttare. Le novità del partito di massa sono collegate, $a$ ) da un lato, all'organizzazione interna, che diviene stabile nel tempo e articolata su base territoriale - le 'sezioni' -, grazie anche al ricorso a funzionari professionalizzati, e $b$ ) dall'altro, ai contenuti veicolati, che si inscrivono in un programma ideologico, indirizzato a una precisa azione politica, e tale da vincolare l'operato degli eletti ${ }^{24}$.

La diffusione dei partiti di massa - ai partiti socialisti faranno rapidamente seguito i partiti popolari e liberali - evidenzia la strutturazione interna delle società dell'epoca: la divisione in classi contraddistinte da interessi diversi e spesso divergenti. In questo modo, i parlamenti nazionali diventano specchio delle rispettive società. Anche per questo motivo, Max Weber poteva vedere nel Parlamento l'istituzione centrale della vita politica, nella quale si confrontano i molteplici valori che pervadono la società $^{25}$. La circostanza che la fisionomia dei partiti rispecchi l'articolazione interna alla società viene solitamente espressa attraverso il concetto di cleavage (frattura) ${ }^{26}$. In altre parole, la struttura del sistema dei partiti rispecchia quella del sistema economico-sociale, con i diversi partiti che rappresentano i settori della società. Nel corso del Novecento, almeno fino agli anni Settanta, questa corrispondenza partiti/società - su cui si basa il successo della democrazia rappresentativa - ha avuto un'importanza cruciale sia nel delineare i contorni del conflitto, sia nello stabilizzarlo, impedendo che si manifestasse in forme distruttive ${ }^{27}$.

L'avvento del partito di massa, se da un lato ha reso possibile l'integrazione politica dei nuovi elettori, dall'altro ha comportato anche il sorgere di problemi inediti. Questi problemi furono diagnosticati, come ho ricordato nel capitolo precedente, nei primi anni del XX secolo da Robert Michels, che, nella sua Sociologia del partito politico, enunciava la legge ferrea delle oligarchie, vale a dire la circostanza secondo la quale il rafforzamento degli apparati di partito comporta una deriva oligarchica: in pratica, i dirigenti prendono il controllo del partito. Ne consegue l'autonomizzazione degli obiettivi perseguiti dalla classe dirigente rispetto

${ }^{24}$ Vedi: Manin, Principî del governo rappresentativo, cit., pp. 229-242; della Porta, I partiti politici, cit., pp. 28-40.

${ }_{25}$ Cfr. Weber, Parlamento e governo, cit.

${ }^{26}$ La formulazione canonica di questa posizione si trova in S.M. Lipset, S. Rokkan, Strutture di clevage, sistemi partitici e allineamenti dell'elettorato, in S.M. Lipset, Istituzioni, partiti, società civile, a cura di L. Morlino, il Mulino, Bologna 2009, pp. 205-237. Il saggio originale di Lipset e Rokkan è del 1967. Sul tema cfr. anche della Porta, I partiti politici, cit., cap. 3.

${ }^{27}$ Vedi della Porta, I partiti politici, cit., pp. 51-55. 
ai fini del partito, con i funzionari che tendono a perpetuare il proprio potere, piuttosto che a perseguire gli obbiettivi del partito stesso, e, più in generale, la perdita del senso di comunità con gli elettori ${ }^{28}$.

\section{Partiti e comunicazione nel secondo dopoguerra}

La fisionomia dei partiti e le modalità della comunicazione politica sono profondamente mutate dalla fine della seconda guerra mondiale a oggi. Già negli anni Sessanta del secolo scorso Otto Kirchheimer elaborava la nozione di partito pigliatutto per descrivere le nuove tipologie di partito che si stavano affacciando sulla scena politica, caratterizzate dalla riduzione del bagaglio ideologico - vale a dire, dei contenuti ideali e progettuali che consentono di situare il partito rispetto ai suoi concorrenti - e dei riferimenti a una specifica classe sociale, dall'aumento del potere dei gruppi dirigenti e da una diminuzione del ruolo degli iscritti ${ }^{29}$.

Le ragioni profonde di questo passaggio sono da ricercare nell'inadeguatezza dei modelli tradizionali di partito rispetto a una serie di fattori di natura economico-sociale. In termini generali, questi fattori possono essere compendiati nelle trasformazioni dell'economia e in particolare nell'archiviazione del modello fordista di produzione, incentrato sulla divisione e la standardizzazione dei compiti, a favore di un modello di produzione post-fordista, basato sull'espansione del settore terziario, trasformazioni che rendevano obsolete le vecchie appartenenze ideologiche e rompevano le tradizionali corrispondenze tra partiti e categorie di lavoratori.

In anni più recenti, i processi di globalizzazione hanno reso questa spaccatura più evidente aprendo nuove linee di conflitto. Come conseguenza, i partiti sono cambiati ancora. Sullo sfondo di una progressiva autonomizzazione del sistema politico, che tende a farsi sempre più autoreferenziale, ${ }^{30}$ di una costante diminuzione del numero degli iscritti e dei votanti e di un aumento della volatilità elettorale, si assiste alla formazione di nuove tipologie di partito, che si innestano su vecchi e nuovi cleavages: i partiti ambientalisti, vale a dire formazioni incentrate sull'attenzione alle tematiche ecologiche ${ }^{31} \mathrm{e}$ i partiti etnoregionalisti, che

${ }^{28}$ Ivi, pp. 58-63. Cfr. anche A. Panebianco, Modelli di partito, il Mulino, Bologna 1982.

${ }^{29}$ Cfr. O. Kirchheimer, La trasformazione dei sistemi partitici dell'Europa Occidentale, in G. Sivini, Sociologia dei partiti politici, il Mulino, Bologna 1979, pp. 243-267. Larticolo di Kirchheimer è del 1966. Cfr. anche della Porta, I partiti politici, cit., cap. 5 .

${ }^{30}$ Cfr. Zolo, Il principato democratico, cit., pp. 142-154.

${ }^{31}$ Vedi della Porta, I partiti politici, cit., pp. 108-112. 
si propongono di difendere gli interessi degli abitanti di un determinato territorio ${ }^{32}$.

Un discorso separato andrebbe fatto in relazione al nesso fra partiti e movimenti sociali. A partire dagli anni Sessanta, come si è detto, i tradizionali partiti di massa entrano in crisi in ragione della loro incapacità di intercettare le istanze provenienti dalla società. La crisi dei partiti è sintomo di una crisi più generale della democrazia parlamentare imperniata sul principio rappresentativo. Dalla fine degli anni Sessanta in poi un'importante alternativa al modello partitico viene dalla diffusione dei movimenti sociali ${ }^{33}$.

I movimenti - che si possono definire come reti di interazione informale, alimentate da credenze condivise, che si mobilitano in azioni di protesta - operano secondo uno schema molto vicino a quello della democrazia diretta, basato sull'azione 'dal basso' e sulla vicinanza agli interessi in gioco. A partire da questa esperienza si è instaurata una dialettica politica che ha visto, soprattutto nella fase iniziale, la vicinanza tra partiti di sinistra e movimenti sociali, ma che, in ultima analisi, ha condotto all'emersione di nuovi tipi di formazione politica - per esempio, il MoVimento 5 Stelle, Podemos, Syriza - che si propongono di superare le forme e le strutture del partito tradizionale - sul piano dei contenuti, della gestione, dell'articolazione territoriale ecc. ${ }^{34}$.

Le trasformazioni relative alla fisionomia dei partiti sono ovviamente connesse con la parallela evoluzione della comunicazione politica, in conseguenza del massiccio impiego, prima della televisione e ora delle piattaforme digitali. In origine, la comunicazione elettorale si rivolgeva quasi esclusivamente agli elettori che già sostenevano il partito e si realizzava tramite affissioni murarie, comizi, incontri, volantinaggio. Quindi, si realizzava in forme selettive, che presupponevano che i cittadini esprimessero nel voto la propria appartenenza ${ }^{35}$.

La situazione si modifica drasticamente con l'ascesa della popolarità della televisione, negli anni Cinquanta negli Stati Uniti e negli anni Sessanta in Europa. In generale, il linguaggio televisivo comporta l'azzeramento delle forme di mediazione tra emittente del messaggio e destinatario. Questa caratteristica induce una ristrutturazione profonda dei rapporti sociali, che si traduce nella tendenziale riduzione dei rapporti orizzontali in favore di quelli verticali e in un consequenziale aumento dell'individualismo. Ciò non può non riflettersi anche sulle modalità in cui avviene la comunica-

${ }^{32}$ Ivi, pp. 113-115.

33 Sul tema, cfr. D. della Porta, M. Diani, I movimenti sociali, La Nuova Italia Scientifica, Roma 1997.

${ }^{34}$ Cfr. della Porta, I partiti politici, cit., cap. 10.

${ }_{35}$ Cfr. G. Cosenza, Semiotica e comunicazione politica, Laterza, Roma-Bari 2018, pp. 30-31. 
zione politica. Le caratteristiche specifiche del mezzo televisivo, unite alle trasformazioni dei partiti privilegiano sempre di più un messaggio di tipo generalista. Il risultato è il prevalere delle forme di comunicazione diretta leader/elettori rispetto alle forme tradizionali che passavano attraverso la mediazione filtrante dei giornali e della discussione interna al partito stesso. Ne segue anche la semplificazione del messaggio politico che tende a farsi slogan, in modo da risultare immediatamente comprensibile e facilmente memorizzabile ${ }^{36}$.

La televisione, inoltre, mette a disposizione dell'emittente alcuni strumenti che possono condizionare la ricezione del suo messaggio. Si tratta, in particolare, del framing, cioè il procedimento con il quale i problemi sono 'incorniciati', suggerendone implicitamente spiegazioni causali e proposte di soluzione e soprattutto dell'effetto agenda, vale a dire, la circostanza secondo la quale la scelta delle notizie da parte delle agenzie di informazione porta con sé un messaggio implicito riguardo alla rilevanza dei temi in discussione, messaggio che si deposita nel ricevente sino a definire una griglia di salienza capace di organizzare la sua attenzione ${ }^{37}$.

Soprattutto, il mezzo televisivo amplifica un processo che già il trapasso dal partito di massa al partito pigliatutto lasciava presagire, vale a dire la tendenza alla personalizzazione della politica, al protagonismo dei leader e delle loro qualità personali ${ }^{38}$. Questa tendenza si coniuga a quella verso l'impiego di classificazioni binarie - giusto/sbagliato, amico/ nemico, noi/loro, vecchio/nuovo ${ }^{39}$ - a definire una traiettoria complessiva che trasforma l'evento politico in una sorta di show allestito per catturare l'attenzione degli elettori. Si parla a questo proposito di spettacolarizzazione della politica ${ }^{40}$.

Negli ultimi dieci anni, peraltro, il messaggio politico si è ulteriormente modificato, in conseguenza dell'avvento del cosiddetto web 2.0 e di tutte le forme di comunicazione informatica che permettono l'interazione tra emittenti e destinatari del messaggio, in primo luogo dei social media. Da un lato, il web - non solo il web 2.0 -, da considerarsi come il luogo nel quale si manifesta oggi la sfera pubblica nel senso di Habermas, permette

${ }^{36}$ Vedi: Zolo, Il principato democratico, cit., pp. 191-195; G. Mazzoleni, La comunicazione politica, il Mulino, Bologna 2012, pp. 43-44; Cosenza, Semiotica e comunicazione politica, cit., pp. 32-33.

37 Vedi Mazzoleni, La comunicazione politica, cit., pp. 217-218. In particolare sull'effetto agenda, vedi Zolo, Il principato democratico, cit., pp. 189-190.

${ }^{38}$ Vedi Mazzoleni, La comunicazione politica, cit., pp. 63-67. Sul tema, cfr. anche: M. Calise, Il partito personale. I due corpi del leader, Laterza, Roma-Bari, 2010; Id., La democrazia del leader, Laterza, Roma-Bari, 2016.

${ }^{39}$ Cfr. Cosenza, Semiotica e comunicazione politica, cit., cap. 1.

40 Vedi Mazzoleni, La comunicazione politica, cit., pp. 56-58. 
a voci che non troverebbero spazio sui canali di comunicazione ufficiali per esempio, la televisione - di farsi udire: in molti casi i social networks hanno costituito una risorsa per organizzare la protesta contro i poteri dominanti, come nel caso delle cosiddette primavere arabe. Questo spiega anche perché Internet è spesso oggetto di censura nei paesi illiberali ${ }^{41}$.

D'altra parte, l'azzeramento dei filtri e dei controlli sulla veridicità delle notizie comporta l'insorgere di numerosi aspetti problematici: il rischio di una permanente sovrapposizione tra realtà e finzione; l'impossibilità di riconoscere le notizie artefatte - le cosiddette fake news -, una caratteristica che può essere facilmente sfruttata a fini manipolatorî, tanto più in quanto anche l'architettura di Internet, basata sull'asimmetria tra i grandi siti, i cosiddetti hubs, che contano milioni di links - siti come Google, Facebook, Microsoft, Apple, Twitter ecc. -, riproduce un assetto nel quale alcune voci riescono a farsi udire molto più di altre; la formazione di cyber-ghetti, all'interno dei quali coloro che condividono certe idee, interpretazioni, valutazioni comunicano esclusivamente fra di loro, radicalizzando il proprio punto di vista e rendendosi impermeabili alle informazioni provenienti dall'esterno ${ }^{42}$.

\section{Partiti e pluralismo}

Dopo questo lungo excursus sulla storia dei partiti e della comunicazione politica, possiamo ritornare sul tema del nesso tra costituzione e pluralismo. Nella prima parte di questo capitolo ho sostenuto che la struttura della democrazia costituzionale riproduce l'assetto dualistico che abbiamo riconosciuto essere tipico della teoria democratica nella dicotomia tra la sfera delle norme costituzionali e quella delle fonti a esse subordinate. Tuttavia, questa struttura si sta evolvendo verso un modello differente, nel quale il livello costituzionale tende a confondersi con quello delle norme sottostanti. Adesso vorrei sostenere che questa torsione è un'altra faccia del percorso compiuto dai partiti politici e, più in generale, dalla democrazia parlamentare.

Come si è visto, nel secondo dopoguerra si comincia ad avvertire l'inadeguatezza dei partiti di massa. Questa inadeguatezza diventa più forte nei decenni successivi, traducendosi nella nascita di nuove tipologie di partito. Soprattutto, essa è radicata nella crescente discontinuità fra le articolazioni ideologiche dei partiti e le fratture socioeconomiche presenti all'interno della società. Si rompe l'isomorfismo tra sistema dei partiti e sistema sociale che fondava il successo della democrazia rappresentativa.

${ }^{41}$ Ivi, pp. 166-168.

${ }^{42}$ Cfr. Sunstein, \#Republic, cit. 
Questo passaggio porta numerose conseguenze: da un lato, si alleggerisce il bagaglio ideologico, dall'altro, in concomitanza con l'evoluzione dei media, il discorso politico tende a personalizzarsi e a spettacolarizzarsi. Queste trasformazioni incidono sul discorso democratico, spostandolo in direzione del potenziamento di tutti quei canali che collegano direttamente cittadini e istituzioni. Come risultato, l'esigenza di rinnovamento che aveva trovato un primo sbocco nella forma dei movimenti sociali e che era stata parzialmente recepita dai partiti di sinistra si è diffusa in tutto lo spettro politico, esprimendosi in partiti e movimenti che, al di là degli schemi ideologici tradizionali, fanno leva sulla vicinanza agli interessi degli elettori e, in nome della difesa di quegli interessi, occupano le istituzioni.

In questo modo, la semplificazione ideologica si traduce, in ultima analisi, nella soppressione del pluralismo che contraddistingueva le costituzioni democratiche. Al dualismo tra Costituzione legge ordinaria, che istituzionalizzava la logica tipica della teoria democratica, succede così un ripiegamento monista entro il quale non c'è spazio per tematizzare lo scarto tra deliberazioni che sono espressione della volontà della maggioranza e deliberazioni 'giuste'. 



\section{Capitolo sesto \\ Crisi e trasformazioni della democrazia}

\section{Elementi di crisi della democrazia}

Di crisi della democrazia si parla ormai da oltre quaranta anni, almeno dalla pubblicazione del famigerato rapporto di Crozier, Huntington e Watanuki alla Commissione trilaterale ${ }^{1}$. Nei decenni che sono seguiti il numero di Stati democratici ha continuato ad aumentare nonostante le peripezie del costituzionalismo moderno e le vicende relative all'evoluzione dei partiti e della comunicazione politica. Non soltanto oltre la metà degli Stati del mondo sono (formalmente) democratici, ma la democrazia sembra rappresentare l'unica forma di governo legittima e la qualifica di 'democratico' rappresenta uno status che i governanti si sforzano di ottenere per accrescere la credibilità internazionale della propria compagine politica. Non si può nemmeno escludere che, mentre il modello democratico si afferma a livello globale come slogan o idea guida, la pratica degli Stati democratici di più antica tradizione si vada corrompendo. Tanto che è difficile anche stabilire se di vera crisi si tratti o degli effetti di una serie di 'condizioni inospitali' che minacciano il fiorire della pianta democratica ${ }^{2}$.

Nondimeno, di crisi della democrazia, come dicevo, si parla da più parti e ormai da diverso tempo, se è vero che già nei primi anni '8o Bobbio poteva parlare di «promesse non mantenute» della democrazia ${ }^{3}$. La

${ }^{1}$ Cfr. Crozier, Huntington, Watanuki, La crisi della democrazia, cit.

${ }^{2}$ Vedi Ferrara, The Democratic Horizon, cit., p. 6.

3 Vedi N. Bobbio, Il futuro della democrazia, in Id., Il futuro della democrazia, Einaudi, Torino 1984, pp. 3-28. Cfr. anche Zolo, Il principato democratico, cit., pp. 121-134.

Leonardo Marchettoni, Breve storia della democrazia: da Atene al populismo, ISBN 978-88-6453760-3 (print) ISBN 978-88-6453-761-o (online PDF) ISBN 978-88-6453-762-7 (online EPUB),

(C) 2018 Firenze University Press 
crisi è motivata, soprattutto in rapporto alle democrazie occidentali ${ }^{4}$, da un lato, dalla perdita di forza propulsiva che l'ideale democratico sembra scontare. Dall'altro, dall'esistenza di criticità di varia origine. Tali criticità possono essere suddivise, per comodità espositiva, in criticità interne allo Stato ed esterne a esso.

Cominciando dalle criticità interne, queste ruotano intorno all'incapacità della politica tradizionale di evadere la domanda di rappresentatività. A sua volta, questo deficit possiede diverse cause che discendono, come sostenevo nel capitolo precedente, dalle trasformazioni del conflitto sociale, in seguito all'avvento del modello economico post-fordista - rese più radicali dalla successiva 'finanziarizzazione' dell'economia - e alla frammentazione dell'insieme dei cittadini in una pluralità di comunità distinte, separate sulla base di profonde spaccature valoriali ${ }^{5}$.

In tutti questi casi, il modello politico tradizionale si dimostra inadeguato. Per una fondamentale ragione: perché le strutture politiche, strutture che informavano e venivano informate dal conflitto, erano predisposte a gestire disaccordi relativi alla distribuzione di beni e di altre risorse scarse, in presenza di un quadro valoriale condiviso. Nel momento in cui tale quadro viene meno - per effetto delle trasformazioni socioeconomiche, della differenziazione culturale e religiosa, dell'incapacità della classe dirigente di immaginare soluzioni inedite ai problemi in gioco - viene meno anche la possibilità di affidarsi con successo alle forme politiche tradizionali.

Il fatto è che le decisioni da prendere negli Stati moderni posseggono solitamente un elevato tasso tecnico. Immediata conseguenza è la riduzione di interesse verso la politica da parte dei cittadini, riduzione che si esprime nella diminuzione della percentuale dei votanti - in Italia si è passati dal 92,2\% delle elezioni politiche del 1948 al 72,9\% del 2018, negli Stati Uniti Donald Trump è stato eletto da circa un quarto degli aventi diritto - e degli iscritti ai partiti. Questo stato di cose solleva dubbi consistenti sull'opportunità di ricorrere sistematicamente al metodo democratico. Infatti, alcuni autori notando i livelli elevati di astensionismo, la disinformazione, l'irrazionalità e la partigianeria dei votanti, hanno caldeggiato il passaggio da forme di governo democratiche a forme di governo epistocratiche, nelle quali, cioè, governano coloro che sono dotati delle conoscenze necessarie ${ }^{6}$.

${ }^{4}$ Nel caso delle democrazie non occidentali il discorso è più complesso, coinvolgendo le specificità storiche e sociali dei diversi Stati.

5 Sull'importanza delle trasformazioni economiche in relazione alla crisi delle democrazie, vedi Ferrara, The Democratic Horizon, cit., pp. 8-9.

${ }^{6}$ Cfr. J. Brennan, Contro la democrazia (ed. or. 2016), Luiss University Press, Roma 2018. Cfr. anche S. Cassese, La democrazia e i suoi limiti, Mondadori, Milano 2017, pp. 36-37. 
Questi fattori si correlano alla cosiddetta regressione oligarchi$c a$, vale a dire, una serie di trasformazioni politiche di medio o lungo periodo che comportano lo spostamento verso l'alto dei centri decisionali, dai cittadini agli eletti, dalla base dei partiti ai leader politici, dai parlamenti agli esecutivi e dagli esecutivi al premier. Si correlano altresì alle trasformazioni della sfera pubblica, in cui la moltiplicazione delle possibilità di interazione comunicativa si accompagna alla sempre maggiore concentrazione della titolarità dei mezzi di comunicazione di massa, alla confusione tra informazione e comunicazione pubblicitaria e, in definitiva, alla tendenziale sovrapposizione di agire comunicativo e agire strategico. La conseguenza è la chiusura degli spazi aperti alla deliberazione in favore delle forme di potere esercitato verticalmente, prescindendo dal confronto ${ }^{7}$.

Per quanto attiene invece alle criticità esterne, queste possiedono varie dimensioni. La prima è legata ancora alla sfera economica. A questo proposito, non solo la globalizzazione dell'economia e la delocalizzazione dei cicli produttivi indeboliscono drasticamente gli strumenti - in primo luogo fiscali - a disposizione degli Stati. I pericoli per la democrazia vengono anche dal fatto che le decisioni in materia di governo delle dinamiche economiche vengono sempre più spesso avocate da organismi e agenzie sovranazionali che dettano agli Stati le politiche da implementare ${ }^{8}$.

La seconda è collegata al modo in cui la regionalizzazione e l'internazionalizzazione della politica, la crescita di reti di comunicazione transnazionali, mettono in questione la funzionalità di un insieme di procedure sorte all'interno dei confini dello Stato Nazione. Qui viene in causa il deficit democratico globale, vale a dire, l'assenza, nelle sedi politiche sovranazionali e internazionali - Unione Europea, ONU -, di meccanismi democratici comparabili con quelli operanti all'interno degli Stati e il prevalere di forme di governance legittimate sulla base di competenze tecniche 9 .

Infine, l'ultima dimensione critica si correla alle cosiddette sfide globali, vale a dire quelle problematiche che - come il terrorismo e la guerra, il riscaldamento globale, il governo delle migrazioni, la crisi economica

7 Sulla regressione oligarchica, vedi Petrucciani, Democrazia, cit., pp. 115-120; cfr. anche Bobbio, Il futuro della democrazia, cit., pp. 10-14. Per una trattazione della sfera pubblica che aggiorna la classica impostazione habermasiana, cfr. A. Honneth, Il diritto della libertà. Lineamenti per un'eticità democratica (ed. or. 2011), Codice, Torino 2015, parte terza.

${ }^{8}$ Su questo punto, si può vedere L. Gallino, Il colpo di Stato di banche e governi. L'attacco alla democrazia in Europa, Einaudi, Torino 2013.

9 Sulla dimensione globale della democrazia, cfr. Ferrara, The Democratic Horizon, cit., cap. 7. Sul tema si può vedere anche: D. Archibugi, Cittadini del mondo. Verso una democrazia cosmopolitica, il Saggiatore, Milano 2009; Cassese, Il diritto globale, cit., cap. VI. 
- per loro natura, non possono essere affrontate efficacemente da singoli Stati ma richiedono forme di cooperazione internazionale ${ }^{10}$.

In tutti questi casi, le decisioni politiche non possono ricevere una piena legittimazione popolare. Ne segue che le forme di governo democratiche soffrono dell'incapacità di gestire i relativi conflitti.

\section{L'alternativa deliberativa}

Gli ultimi anni dello scorso secolo e l'inizio del secolo successivo hanno visto la progressiva diffusione di tipologie di conflitto non facilmente incanalabili nelle strutture politiche tradizionali, determinando un deficit di rappresentatività e più in generale la crescente inadeguatezza del modello democratico, spesso insidiato da forme di governance tecnocratica. Anche in risposta a queste criticità, nella seconda metà del secolo scorso si sono teorizzate e sperimentate nuove forme di coinvolgimento degli elettori nei meccanismi democratici. Si parla di democrazia partecipativa per indicare quelle architetture istituzionali nelle quali la partecipazione effettiva dei cittadini è assicurata non soltanto in occasione delle consultazioni elettorali ma più in generale nelle attività di governo - in particolare per quanto riguarda il governo locale - l'organizzazione del lavoro, il sistema dei partiti1 ${ }^{11}$.

Dagli anni Ottanta del secolo scorso si inizia a parlare di democrazia deliberativa. Tra gli autori coinvolti, oltre ad Habermas, merita qui ricordare Bernard Manin, Joshua Cohen, Seyla Benhabib, James Fishkin, John Dryzek $^{12}$. Il nucleo dell'approccio deliberativo consiste nell'idea secondo la

${ }^{10}$ Sulle sfide globali, cfr. F. Cerutti, Sfide globaliper il leviatano. Una filosofia politica delle armi nucleari e del riscaldamento globale, Vita \& Pensiero, Milano 2009.

${ }^{11}$ Sul tema, cfr.: C. Pateman, Participation and Democratic Theory, Cambridge University Press, Cambridge 1970; C.B. Macpherson, La vita e itempi della democrazia liberale (ed. or. 1977), il Saggiatore, Milano 1980; Y. Sintomer, Il potere al popolo. Giurie cittadine, sorteggio e democrazia partecipativa (ed. or. 2007), Dedalo, Bari 2009; U. Allegretti (a cura di), Democrazia partecipativa. Esperienze e prospettive in Italia e in Europa, Firenze University Press, Firenze 2010.

${ }^{12}$ Cfr.: B. Manin, On Legitimacy and Political Deliberation, «Political Theory», 15 (1987), pp. 338-368; J.S. Fishkin, Democracy and Deliberation: New Directions for Democratic Reforms, Yale University Press, New Haven 1991; Habermas, Fatti e norme, cit., cap. 7; S. Benhabib, Toward a Deliberative Model of Democratic Legitimacy, in Ead. (ed.), Democracy and Difference, Princeton University Press, Princeton 1996, pp. 67-94; J. Cohen, Procedure and Substance in Deliberative Democracy, in S. Benhabib (ed.), Democracy and Difference, cit., pp. 95-119; J. Bohman, Public Deliberation: Pluralism, Complexity, and Democracy, Mit Press, Cambridge 1997; J.S. Dryzek, Deliberative Democracy and Beyond: Liberals, Critics, Contestations, Oxford University Press, Oxford 2000; J.S Fishkin, When the People Speak: Deliberative Democracy and Public Consultation, Oxford University Press, Oxford 2009; J.S. Dryzek, Foundations and Frontiers of Deliberative Governance, Oxford 
quale il limite principale delle concezioni tradizionali della democrazia - le cosiddette concezioni aggregative - consiste nell'assunzione secondo la quale le preferenze dei cittadini sono già preformate e immutabili rispetto al momento del voto. Questo punto di partenza non è molto differente da quello degli argomenti, di matrice elitista, secondo i quali il momento elettorale segna l'evento finale di una sorta di competizione per il voto popolare, competizione nella quale i ruoli attivi sono appannaggio di una ristretta oligarchia.

Il modello deliberativo rappresenta un tentativo di reagire a quelle stesse criticità che già gli elitisti avevano diagnosticato. I deliberativisti sottolineano la centralità del momento della decisione, momento nel quale si formano realmente le opinioni in gioco e ritengono che siano 'valide' soltanto quelle deliberazioni raggiunte seguendo procedure 'corrette'. Il limite delle democrazie contemporanee consiste nel fatto che le scelte politiche degli elettori sono il frutto di una discussione per lo più superficiale e scarsamente informata, entro una sfera pubblica saturata dalla propaganda dei media e dai sondaggi di opinione. Per ovviare a questo problema le regole procedurali dovrebbero essere definite in modo da non permettere il formarsi di volontà non distorte, nel senso che la scelta di quelle regole deve poter garantire la bontà degli esiti della deliberazione ${ }^{13}$.

L'approccio deliberativo è presentato da alcuni suoi sostenitori come una riscoperta delle origini classiche della democrazia, basate sul confronto dialogico ${ }^{14}$. Allo stesso modo in cui ad Atene le decisioni vitali venivano prese dopo essere state discusse fra tutti i cittadini, così adesso bisognerebbe passare da un modello nel quale i cittadini possono solo scegliere tra una serie di alternative già preconfezionate a uno nel quale viene riscoperta la centralità della fase di discussione e definizione delle opzioni in campo. Ci sono però buoni motivi per essere scettici rispetto a questo modo di presentare le cose, dal momento che, come abbiamo visto nel primo capitolo, il sistema democratico ateniese si risolveva in una forma di governo nella quale il potere era gestito dagli esponenti delle famiglie aristocratiche attraverso il supporto popolare.

Anche alla luce di questo rilievo, sembra più opportuno caratterizzare il nucleo della democrazia deliberativa in termini habermasiani, cioè in quanto definizione di un ambito di agire rivolto all'intesa. I teorici deliberativisti sottolineano che l'essenza delle procedure democratiche consiste nella predisposizione di sedi di discussione, il più possibile libere da condizionamenti di tipo strategico e tali da permettere l'ascolto

University Press, Oxford 2010. In italiano, cfr. A. Floridia, Un’idea deliberativa della democrazia. Genealogia e principî, il Mulino, Bologna 2017.

${ }_{13}$ Vedi Fishkin, Democracy and Deliberation, cit., cap. 1.

${ }_{14}$ Vedi Fishkin, When the People Speak, cit., pp. 9 sgg. 
delle ragioni dell'altro. L'attivazione di questi canali di comunicazione dovrebbe consentire, da un lato l'esposizione in forma discorsiva delle convinzioni - e quindi, in un certo senso, la conversione dell'identità in discorsi -, dall'altro, la partecipazione alla decisione da parte di tutti i soggetti potenzialmente interessati ${ }^{15}$.

Riguardo a quest'ultimo punto, alcuni autori, seguendo Habermas, hanno sostenuto che la discussione deve raggiungere l'obiettivo di una valutazione imparziale, inclusiva e non faziosa delle questioni in oggetto. La deliberazione deve comportare l'acquisizione di informazioni, l'apprezzamento delle relative conseguenze, risolvendosi nella verifica di soluzioni e giustificazioni, piuttosto che nella costruzione di principî universali ${ }^{16}$. Altri autori, tuttavia, hanno criticato il punto di vista 'imparzialista' sostenendo che rappresenta un ideale inattingibile ${ }^{17}$, oppure rilevando che solitamente gli attori del dibattito presentano la loro proposta in modo da farne risaltare l'utilità general $\mathrm{e}^{18}$, oppure ancora che i gruppi dominanti fissano le regole dell'argomentazione, regole che possono scoraggiare la partecipazione di alcuni soggetti interessati ${ }^{19}$.

Passando dal versante teorico a quello operativo, un breve cenno meritano le soluzioni istituzionali ideate dai teorici deliberativisti. In generale, i sostenitori della democrazia deliberativa, sottolineano l'importanza dell'educazione alla cittadinanza. I deliberativisti pongono grande enfasi sul ruolo dell'informazione puntuale intorno alle politiche intraprese. L'informazione può essere ottenuta tramite l'impiego di piattaforme digitali - il cosiddetto e-government - piattaforme che, peraltro possono venire in causa anche per quanto riguarda l'acquisizione degli orientamenti dell'elettorato. Si parla in questo caso di procedure di e-democracy ${ }^{20}$.

Tuttavia, l'iniziativa più caratteristica riguarda la previsione di giornate deliberative, vale a dire appuntamenti dedicati all'informazione e alla discussione, da parte di campioni rappresentativi di elettori, di proposte concrete di interesse pubblico, con il coordinamento di esperti neutrali,

${ }^{15}$ Vedi, per esempio, Habermas, Fatti e norme, cit., pp. 360 sgg.

${ }^{16}$ Cfr. Benhabib, Toward a Deliberative Model of Democratic Legitimacy, cit.

${ }^{17}$ Cfr. A. Gutman, D. Thompson, Democracy and Disagreement, Harvard University Press, Cambridge, Mass. 1997.

${ }^{18}$ Cfr. J. Elster, The Market and the Forum: Three Varieties of Political Theory, in J. Bohman, W. Rehg (eds.), Deliberative Democracy, Mit Press, Cambridge 1997, pp. 3-34.

${ }_{19}$ Cfr. J. Tully, The Unfreedom of the Moderns in Comparison to their Ideals of Constitutional Democracy, in Id., Public Philosophy in a New Key, vol. 2: Imperialism and Civic Freedom, Cambridge University Press, Cambridge 2008, pp. 91-123. Su democrazia e inclusione cfr. anche I.M. Young, Inclusion and Democracy, Oxford University Press, Oxford 2000.

${ }^{20}$ Sul tema, cfr. G. Gometz, Democrazia elettronica. Teoria e tecniche, Ets, Pisa 2017. 
secondo procedure mirate a includere tutte le posizioni sul tema. Eventi di questo tipo si sono verificati più volte negli Stati Uniti, sotto l'auspicio di studiosi come Bruce Ackerman e il già citato James Fishkin ${ }^{21}$. Si è riscontrato che i soggetti che avevano partecipato al dibattito modificavano tendenzialmente le proprie opinioni di partenza, con risultati differenti in relazione alla composizione del campione di elettori con il quale erano entrati in contatto. In ogni caso, è opportuno sottolineare, che un ruolo cruciale in rapporto agli esiti, è detenuto da coloro che coordinano le attività di informazione e discussione ${ }^{22}$.

In definitiva, il nucleo dell'approccio deliberativo, con la sua enfasi sul momento procedurale, conferma l'ipotesi che ha guidato questa ricostruzione: il modello deliberativo rappresenta il tentativo di rimodulare il familiare assetto a due livelli, che cerca di arginare il rischio che la maggioranza produca deliberazioni 'ingiuste', tramite un'accurata selezione delle procedure 'corrette'. In ciò segue una tendenza tipicamente novecentesca, da Weber, a Schumpeter, fino ad Habermas. Il restringimento all'ambito delle procedure doveva fronteggiare la frantumazione dei sistemi valoriali e il conseguente l'aumento del pluralismo assiologico. Il problema è che la definizione di un meccanismo procedurale 'corretto' si è rivelata un'impresa pressoché impossibile, e anche se essa fosse finalmente realizzata, questo risultato non garantirebbe la 'correttezza' dei contenuti oggetto della deliberazione.

\section{Populismo}

Se gli approcci deliberativi costituiscono un tentativo di reagire alle criticità della democrazia rivitalizzandone le strutture, l'ascesa dei partiti cosiddetti 'populisti' ne rappresentano, per così dire, una conseguenza di segno opposto. Bisogna chiarire subito che 'populismo' è un termine cui manca un referente empirico univoco e che ammette diverse instanziazioni nel tempo e nello spazio. Si parla di populismo a proposito del People's Party ${ }^{23}$, una formazione politica statunitense dell'inizio del XX secolo, di alcune esperienze politiche sudamericane di ispirazione progressista, come dei partiti di estrema destra dell'Europa contemporanea ${ }^{24}$.

${ }^{21}$ Cfr.: B. Ackerman, J.S. Fishkin, Deliberation Day, Yale University Press, New Haven 2004; J.S. Fishkin, Democracy When the People Are Thinking: Revitalizing Our Politics Through Public Deliberation, Oxford University Press, Oxford 2018.

${ }^{22}$ Cfr. anche C.R. Sunstein, A cosa servono le Costituzioni. Dissenso politico e democrazia deliberativa (ed. or. 2001), il Mulino, Bologna 2009.

${ }^{23} \mathrm{Su}$ cui cfr. M. Kazin, The Populist Persuasion: An American History, Basic Books, New York 1998.

${ }^{24}$ Nelle pagine che seguono ho ripreso alcune parti di L. Marchettoni, Teorie del populismo, «Jura Gentium», XIV (2), 2017, pp. 161-171. 
Secondo un approccio comune il populismo si può definire come un'ideologia thin, basata sulla contrapposizione tra il popolo puro e le corrotte élite e sul nesso tra politica e volontà generale ${ }^{25}$. Da questo punto di vista, il populismo è una forma di reazione a un complesso di fattori - in cui l'aumento delle diseguaglianze economiche gioca un ruolo preponderante - che ha esacerbato il ceto medio, deprimendone l'autopercezione. Pertanto, non è un 'ismo' come gli altri - 'totalitarismo', 'nazionalismo', 'socialismo' ecc. - , non si può cercare di definirlo ammassando una lista di criteri distintivi che servono a identificarne gli esempi. Di conseguenza, l'insieme dei presunti partiti populisti è molto vasto.

Altri autori, tuttavia, ritengono che non sia sufficiente essere critici delle élite per essere populisti. È necessario almeno far valere un atteggiamento anti-pluralista. Perciò, la politica populista è sempre una politica delle identità, che contrappone un concetto artificiale di popolo a un 'nemico' ugualmente fantomatico, moralizzando la contrapposizione. L'assunzione di base è che i partiti populisti intercettano un disagio autentico e che coloro che decidono di votarli sono spinti da ragioni e non agiscono solo in base alle proprie emozioni. Il problema principale è dato dal deficit rappresentativo della democrazia contemporanea. A questo deficit i populisti rispondono in maniera peculiare. I populisti non avversano necessariamente la rappresentanza, né le istituzioni, anche se, essendo anti-pluralisti, tendono a occupare queste ultime e a distorcere il concetto di rappresentanza in modo da trasformarla in rappresentanza simbolica, in cui il partito populista si erge a rappresentante dell'intero popolo ${ }^{26}$.

Questa torsione richiede che venga azzerato lo spazio riservato ai corpi intermedi, in modo da stabilire un contatto immediato tra il leader e i suoi elettori ${ }^{27}$. Il risultato non è tanto la trasformazione della democrazia in una 'democrazia illiberale'. Si tratta piuttosto di una lesione agli stessi meccanismi democratici, che richiedono un'effettiva libertà di opinione e di riunione, un reale pluralismo nei media, leggi a tutela delle minoranze ecc. ${ }^{28}$. Da questo punto di vista, anche fascismo e nazismo sono forme di

${ }^{25}$ Cfr.: C. Mudde, C. Rovira Kaltwasser, Populism: A Very Short Introduction, Oxford University Press, Oxford 2017; M. Revelli, Populismo 2.o, Einaudi, Torino 2017.

${ }^{26}$ Vedi Müller, Cos'è il populismo?, cit., p. 7. Cfr. anche la complessa elaborazione di questo tema svolta da Ernesto Laclau: E. Laclau, La ragione populista (ed. or. 2005), Laterza, Roma-Bari 2008, specialmente il cap. 4.

${ }_{27}$ Su questo punto, cfr. T. Boeri, Populismo e Stato sociale, Laterza, Roma-Bari 2017.

${ }^{28}$ Nadia Urbinati parla a questo proposito di «democrazia sfigurata». Cfr. N. Urbinati, Democrazia sfigurata. Il popolo tra opinione e verità (ed. or. 2014), Università Bocconi Editore, Milano 2017. 
populismo - anche se non sono solo quello. I movimenti e i partiti progressisti anti-establishment invece non lo sono ${ }^{29}$.

Altri autori ancora si focalizzano, invece, sulla dimensione comunicativa, collegando populismo e social media ${ }^{30}$. Secondo questo punto di vista, l'avvento del web 2.0 ha rivoluzionato la scena della politica, facendo esplodere l'imprevedibilità elettorale. Il mezzo digitale consente infatti la costruzione di un popolo che non c'è e che forse non è mai esistito. Questo popolo virtuale, poi, si presta ai tentativi di trasferire l'indignazione contro le élite che lo hanno impoverito sui bersagli selezionati dal leader populista.

Questi tre approcci si mostrano tutti insoddisfacenti. Al primo potremmo obiettare di fornire una caratterizzazione di populismo debole, che permette di mettere insieme soggetti eterogenei. Al secondo, potremmo obiettare, al contrario, di offrire una presentazione troppo selettiva, che conduce a una definizione parziale del fenomeno - il partito populista diventa una sorta di 'partito fascista non violento'. Infine, il terzo approccio si dimostra discutibile soprattutto perché, da un lato, le trasformazioni della comunicazione politica - come l'aumento della volatilità elettorale - sono inscrivibili, come abbiamo visto, in un tendenza di lungo periodo che ha origine ben prima dell'ascesa dei partiti populisti, dall'altro, perché non tutti i partiti populisti fanno ugualmente ricorso ai media digitali.

La conclusione che vorrei trarre da questo excursus è la seguente. Quello di populismo è un concetto ibrido, una vera chimera teorica, nata dalla giustapposizione di diversi profili, la cui analisi andrebbe condotta, per quanto possibile, in modo indipendente, così da arrivare alla costruzione di una sorta di 'populismo idealtipico'. Si potrebbe pensare di indicare alcuni di questi punti nella: a) struttura del partito e nello stile della comunicazione politica; b) nel rapporto più o meno oppositivo verso l'establishment; c) nel grado di autoritarismo.

Per vedere il nesso esistente tra questi tre aspetti, può essere utile collocarli in una prospettiva storica. Che la fisionomia dei partiti e le modalità della comunicazione politica siano profondamente mutate dalla fine della seconda guerra mondiale a oggi non dovrebbe essere una novità. Come ho cercato di illustrare nel capitolo precedente, il progressivo svuotamento ideologico che ha segnato i partiti nella seconda metà del secolo scorso, unito alla crisi della democrazia rappresentativa ha aperto il campo a una serie di tentativi di recupero delle logiche tipiche della democrazia diretta, declinate in rapporto alla vicinanza ai nuovi interessi che la politica tradizionale aveva lasciato senza rappresentanza. Queste

29 Vedi Müller, Cosè il populismo?, cit., p. 125.

${ }^{30}$ Cfr.: A. Dal Lago, Populismo digitale. La crisi, la rete e la nuova destra, Raffaello Cortina, Milano 2017; Y. Mounk, Popolo vs democrazia. Dalla cittadinanza alla dittatura elettorale (ed. or. 2018), Feltrinelli, Milano 2018, pp. 130-140. 
trasformazioni sono ovviamente connesse con la parallela evoluzione della comunicazione, testimoniata dal massiccio impiego, prima della televisione e ora delle piattaforme digitali. Solo che questa evoluzione ha proceduto con velocità diverse in realtà sociali e politiche differenti, di modo che il protagonismo personalistico dei leader si è valso di diversi strumenti ${ }^{31} \mathrm{e}$, d'altra parte, la messa a profitto delle possibilità offerte dalle nuove tecnologie ha contraddistinto pressoché tutti gli orientamenti ${ }^{32}$. Il risultato complessivo è una tendenza generale verso la personalizzazione, la spettacolarizzazione e la disintermediazione della politica. Se si vuole, si può parlare a questo proposito dell'ascesa di una logica populista o di uno stile populista ${ }^{33}$.

L'orientamento anti-establishment è correlato in vari modi a questo processo, ma non va confuso con esso. L'opposizione alle élite è un fattore ricorrente nella storia della politica, almeno dal People's Party in poi. Chiaramente, sull'orientamento politico un peso decisivo lo esercitano fattori di natura socioeconomica. Su questo punto tutti gli studiosi considerati sono concordi nell'elencare i principali responsabili: le promesse mancate della democrazia; la crisi di rappresentatività dei partiti tradizionali; la crescita delle disuguaglianze; l'incapacità delle strutture dello Stato sociale, già messe a dura prova dall'ascesa delle ricette neoliberali, di integrare nei ranghi della politica tradizionale le schiere dei lavoratori impoveriti dalla globalizzazione dei cicli produttivi e dei capitali34.

D'altra parte, nel momento in cui il bagaglio ideologico si assottiglia la polarizzazione del discorso e la costruzione di una opposizione fra un 'Noi' fittizio e un 'Altro' non meno artificiale si dimostra funzionale al progetto politico, perché contribuisce, escludendo tutti i fattori di disturbo, a ridurre la complessità sociale, consentendo una migliore gestione della paura ${ }^{35}$. In questa situazione diventa anche difficile non oltrepassare, sull'onda di un'attitudine che porta gli esponenti del partito populista a identificare

${ }^{31}$ A questo proposito, si deve notare che le strategie di populisti conclamati come Recep Tayip Erdoğan, Viktor Orbán o il leader polacco Jaroslav Kaczyński puntano molto di più sull'occupazione dei media tradizionali che sul digitale.

${ }^{32}$ Il primo Presidente degli Stati Uniti e il primo leader politico di spicco che abbia puntato massicciamente su Internet per raccogliere consensi, utilizzando una strategia che integrava sapientemente mezzi di comunicazione tradizionali e nuove tecnologie, è stato Barack Obama nel 2008. Vedi Cosenza, Semiotica e comunicazione politica, cit., pp. 35-37.

${ }_{33}$ Cfr.: Laclau, La ragione populista, cit., p. 111; B. Moffitt, The Global Rise of Populism: Performance, Political Style, and Representation, Stanford University Press, Stanford 2016.

${ }^{34}$ Cfr.: Revelli, Populismo 2.o, cit., pp. 6-7; Müller, Cos’è il populismo?, cit., pp. 102-106; Mounk, Popolo vs democrazia, cit., capp. 1-3. Specificamente sul tema delle disuguaglianze, il riferimento obbligato è Th. Piketty, Il capitale nel XXI secolo (ed. or. 2013), Bompiani, Milano 2014.

35 Vedi Zolo, Il principato democratico, cit., p. 77. 
sé stessi con l'intero corpo politico, il discrimine del rispetto del rule of law e del modello liberale di democrazia.

In definitiva, l'ascesa del populismo rappresenta l'esito di una vicenda evolutiva più vasta, che interessa sia la trasformazione delle strutture politiche e della comunicazione, sia l'evoluzione dei rapporti tra sistema politico e società. L'altro polo di questa vicenda è rappresentato, però, dalla metamorfosi degli elettori: sono gli elettori, infatti, che si lasciano sedurre da ricette troppo semplici, ricette che sembrano compendiare le convinzioni, di senso comune, che essi stessi intrattengono.

Nella misura in cui gli elettori accettano le semplificazioni populiste, ricadono in un meccanismo che già Tocqueville ne La democrazia in America aveva colto. Tocqueville aveva descritto la passione per l'uguaglianza che caratterizza le società democratiche e travolge tutte le differenze di status tipiche delle società pre-rivoluzionarie ${ }^{36}$. La passione per l'uguaglianza, genera l'individualismo e il conformismo che, a loro volta, costituiscono la precondizione per lo stabilirsi di una nuova forma di dispotismo ${ }^{37}$. Ebbene, le società contemporanee sembrano presentare uno scenario nel quale la passione per l'uguaglianza si esprime sul piano cognitivo, mettendo in dubbio la forza delle argomentazioni e cancellando le gerarchie costruite sulla base delle competenze riconosciute.

In altre parole, quello che viene meno è il riconoscimento dell'autorità epistemica, sostituita da un rapporto organico di appartenenza. Ma in questo modo, vengono meno anche le precondizioni di una comunicazione razionale, rimpiazzate da una relazione di tipo fideistico, di cui la sostituzione della verità con la postverità è la migliore epitome ${ }^{38}$. Per questo motivo, le semplificazioni trovano credito, perché corrispondono all'idea secondo la quale le soluzioni più semplici e più 'autentiche' sono quelle che colgono il cuore dei problemi, al di là delle complicazioni tendenziose elaborate dai 'tecnici' e dai veterani della politica. Si alimenta così un fiume di pregiudizi, fake news, banalizzazioni di questioni ben altrimenti complesse - fiume che i media digitali sono i più atti a incanalare -, che travolge l'argine delle specializzazioni e alimenta le derive autoritarie.

${ }^{36}$ Vedi Tocqueville, La democrazia in America, cit., vol. II, parte II, cap. 1.

37 Ivi, parte IV, cap. 6 .

${ }^{38}$ Con il termine 'postverità' - calco di post-truth, che però è usato prevalentemente come aggettivo, es. post-truth world, mentre 'postverità' ricorre sia come aggettivo che come sostantivo - si qualifica una condizione nella quale la verità riveste un'importanza secondaria rispetto alla credenza diffusa e all'adesione emotiva oppure si indica la proprietà di un fatto o una notizia per cui esso o essa, pur non essendo necessariamente vero/a, riceve un vasto credito. Sul tema, cfr.: M. Ferraris, Postverità e altri enigmi, il Mulino, Bologna 2017; W. Quattrociocchi, A. Vicini, Liberi di crederci. Informazione, internet e post-verità, Codice, Torino 2018; A.M. Lorusso, Postverità, Laterza, Roma-Bari 2018. 



\section{Conclusioni}

\section{Democrazia e verità}

Come ho sostenuto nel capitolo precedente il populismo costituisce l'antitesi della democrazia deliberativa. Nel senso che, come l'approccio deliberativo riproponeva, sublimandola, la struttura a due livelli tipica del discorso democratico novecentesco, così l'atteggiamento populista si coniuga a un quadro istituzionale monista, nel quale la parte al potere assomma su di sé la rappresentanza di tutto il popolo e si esprime attraverso documenti normativi che ignorano la distinzione tra costituzione e fonti a essa subordinate.

Rilevare questa torsione non implica necessariamente giudicare che la svolta sia censurabile. Dopo tutto, la teoria della democrazia, che ho cercato di ripercorrere in questo testo, ha sempre comportato, da Aristotele in poi, l'esclusione di voci, istanze, soggetti. La teoria aristotelica escludeva le donne, gli stranieri e gli schiavi dalle attività di governo, collegando partecipazione politica e differenze naturali. Nel Medioevo e nella prima modernità, il discrimine si determina in relazione alla condizione socioeconomica, sulla base dell'appartenenza a corpi intermedi, come le corporazioni. Questo stato di cose è contraddetto solo in apparenza dall'ascesa del modello contrattualista, che assume la naturale uguaglianza degli individui, dal momento che tale modello, almeno nella sua variante lockiana, si coniuga alla ricezione dell'ordine sociale esistente.

È importante sottolineare che gli effetti di esclusione connessi con il modello democratico rappresentano, per così dire, l'altra faccia della medaglia rispetto al tentativo di immunizzare i processi democratici dallo spettro della tirannide della maggioranza. Il problema da cui muove la riflessione di Aristotele è quello di dimostrare, contro Platone, che anche un vasto insieme di persone dispone delle capacità necessarie per governare, pertanto un governo 'popolare' non si sarebbe risolto inevitabilmente in un regime 'disordinato', in cui il popolo persegue unicamente 
i propri interessi. Chiaramente, la definizione dei criteri normativi che selezionano i processi democratici 'giusti' possiede uno spessore politico, tendendo a giustificare l'assetto vigente. Ma, in ogni caso, non sembra facile separare i due profili. Così sarà anche nei secoli successivi con la teoria democratica a inseguire la struttura sociale, fino alla proposizione di un prototipo di cittadino democratico esemplato sul modello del maschio bianco proprietario.

La vicenda rivoluzionaria consacra questo assetto, che si consolida nell'Ottocento con l'ascesa della classe borghese e la rimodulazione dei dispositivi di esclusione su base censitaria. Nel corso del secolo successivo, l'introduzione del suffragio universale forza una nuova torsione: mentre la teoria democratica viene riformulata in termini procedurali per poi confluire nell'alveo del costituzionalismo, i criteri normativi che presiedono alla regolazione dei processi democratici si dispongono sulla duplice linea dei requisiti formali e dei contenuti sostanziali. Nell'ambito della teoria politica questi due versanti vengono sintetizzati nella proposta di Habermas, che rivendica lo spessore etico delle procedure democratiche.

Naturalmente, una teoria normativa della democrazia - come ogni altra teoria normativa - non può non realizzare un'opera di selezione. Altrimenti, verrebbe meno alla sua funzione. Il problema è contemplare soltanto quelle esclusioni che sono veramente 'giuste'. Ma per effettuare questa valutazione è necessaria una teoria anteriore che giustifichi appunto le scelte compiute. Da questo punto di vista, se guardiamo alle teorie di Aristotele, Locke, Mill - per citare solo alcuni nomi - ci appaiono gravemente insoddisfacenti, infarcite di assunzioni e pregiudizi discutibili che conducono a una scelta su base antropologico-sociale dei cittadini. D'altra parte, le teorie di autori come Spinoza, Rousseau, Marx, che rifiutano l'impianto 'aristocratico', riconducibile al paradigma del governo misto, e quindi sono meno suscettibili di produrre effetti di esclusione, non offrono indicazioni su come impostare la questione del rapporto tra democrazia e giustizia che non siano generiche esortazioni alla realizzazione del bene comune.

Più soddisfacente appare l'assetto dualista che si è realizzato nella seconda metà del Novecento con le democrazie costituzionali. Tuttavia, anche questo tentativo, non è esente da difficoltà. Il problema principale risiede nel fatto che quel modello richiede inevitabilmente, per la propria implementazione, il funzionamento di enti - come le Corti, costituzionali e internazionali, come, su un diverso piano, gli organismi di governance sovranazionali - che si legittimano sulla base della propria supposta competenza tecnica, non su base democratica, e quindi perdono ogni credibilità nel momento in cui il principio di autorità epistemica viene a cadere. L'esito, in presenza di un contesto di crisi economica, è l'ascesa di schieramenti e forze politiche che pretendono di contrapporre all'autorità epistemica l'autorialità del demos, intendendo con questa espressione 
il principio secondo il quale l'unica fonte di legittimità risiede nella riconducibilità alla volontà popolare. Sta di fatto, però, che la conseguente chiusura particolarista e identitaria, avocata dai partiti populisti, a fronte dell'apertura raggiunta all'interno del modello democratico-costituzionale, suona come un'involuzione.

L'aspetto paradossale risiede in questo: i partiti populisti si pongono l'obiettivo di superare i limiti di rappresentatività dei partiti tradizionali, aspirando a farsi portavoce dell'intero corpo elettorale. Solo che i tratti definitori del popolo invocato dai populisti corrispondono a una comunità inesistente. Per tale motivo, il tentativo di proporre una forma di democrazia 'non-liberale' si risolve nella drammatica esclusione di tutti i soggetti che non esibiscono i tratti distintivi del cittadino prototipico, costruito attraverso la proiezione dei tratti dell'elettorato di riferimento. $\mathrm{E}$ del resto, tale operazione soddisfa un basilare principio di riequilibrio, in quanto ottempera all'obiettivo di riduzione della complessità sociale propria dei sistemi politici in generale, come notavo nel capitolo quinto. In risposta all'aumento di complessità sociale successivo alla fine della guerra fredda, complessità di fronte alla quale le democrazie costituzionali si presentavano impreparate, il ripiegamento monista e identitario assolve la funzione di rassicurare i cittadini eliminando i fattori potenzialmente disturbanti.

L'ascesa dei partiti populisti sembra minacciare le promesse di maggiore inclusione della democrazia moderna. Dal momento, però, che il loro successo è radicato negli stessi meccanismi che regolano il funzionamento dei sistemi politici, non è chiaro in che modo essa possa essere contrastata. Molti autori ritengono che la deriva populista possa essere arrestata soltanto attraverso una paziente opera di 'smontaggio', colpendo le semplificazioni e le falsità su cui si basano le ricette populiste. Vi sono ragioni, tuttavia, per essere meno ottimisti. Altri studiosi hanno sostenuto che una strategia argomentativa è inefficace perché banalmente coloro che si lasciano persuadere dalle semplificazioni populiste non si fanno convincere da una confutazione razionale dei presupposti su cui quelle semplificazioni si reggono ${ }^{1}$. Questo esito appare tanto più scontato quanto più si riconosca la funzione 'rassicurante' che quelle semplificazioni svolgono.

Ma più radicalmente, non è semplice neppure capire in che cosa una supposta confutazione potrebbe consistere. Per chiarire questo punto, ipotizziamo che una certa scelta politica venga motivata adducendo certi dati, notizie e teorie. Gli oppositori possono contrastare questa misura

${ }^{1}$ Vedi: Ferraris, Postverità e altri enigmi, cit., pp. 60-61; Quattrociocchi, Vicini, Liberi di crederci, cit., pp. $115 \mathrm{sgg}$. Sugli equivoci del fact checking, cfr. Lorusso, Postverità, cit., pp. 53 sgg. 
cercando di mostrare che gli uni e le altre sono stati falsificati. Ma per fare ciò dovranno necessariamente appoggiarsi ad altri dati, altre notizie e altre teorie, che, a loro volta, potranno essere etichettate come fake news dai loro avversari. Si genera così una spirale di accuse e controaccuse in cui non si riesce a separare realtà e finzione.

Per questi motivi, una risposta costruttiva alla propaganda populista non può passare attraverso il tentativo di smascherarne le semplificazioni ma dovrebbe in qualche modo andare alla radice del problema, investendo le dinamiche stesse di riduzione della complessità sociale che essa mette in atto. Ciò comporta anche ripristinare l'autorità epistemica del discorso pubblico. Ma questo, a sua volta, significa ristabilirne la capacità di operare un'efficace selezione dei rischi, riducendo la complessità sociale e l'insicurezza.

Il tema dell'autorità epistemica del discorso pubblico ci porta a riflettere conclusivamente sul ruolo della verità in democrazia ${ }^{2}$. Infatti, in tanto il discorso pubblico può ambire a essere epistemicamente autorevole in quanto l'autorità epistemica si appoggi sulla capacità di produrre affermazioni vere. Nell'ambito politico ciò implica che i governanti devono poter fare affermazioni che fanno presa sulla realtà, affermazioni che i governati possono riconoscere come veridiche e quindi capaci di ridurre l'incertezza.

Ma il concetto di verità presenta alcune complicazioni che non possono essere ignorate, nemmeno a un'analisi superficiale. Tradizionalmente si distingue tra due concezioni principali della verità: la concezione corrispondentista e la concezione coerentista ${ }^{3}$. La concezione corrispondentista è la più antica e può essere riassunta dicendo che la verità consiste in una forma di corrispondenza tra i nostri discorsi e come il mondo realmente è. «La neve è bianca» è un'affermazione vera se, e solo se, la neve è bianca. Benché a prima vista inattaccabile, questa concezione incorre in molteplici difficoltà: come è possibile stabilire una corrispondenza tra le parole e le cose? In che modo le proposizioni di senso comune - per esempio: «Il tavolo è rotondo» - possono essere dette vere se le teorie scientifiche più accreditate fanno affermazioni diverse (e molto più complesse: per esempio, in termini di atomi situati nello spazio)?4

Problemi come questi motivano l'adesione a teorie di tipo coerentista, basate cioè sulla coerenza complessiva delle affermazioni ritenute vere entro un certo contesto (o all'interno di una certa comunità). D'altra parte, le teorie coerentiste danno l'impressione di un girare a vuoto che prescinde da un contatto con il mondo: se tutto ciò che conta è la coerenza

${ }^{2}$ Su verità e democrazia, cfr.: J. Nida-Rümelin, Democrazia e verità (ed. or. 2006), FrancoAngeli, Milano 2015; Ferrara, The Democratic Horizon, cit., cap. 8.

${ }^{3}$ Per una introduzione sul tema, cfr. G. Volpe, La verità, Carocci, Roma 2012.

${ }^{4}$ Ivi, cap. 2. 
di un sistema di proposizioni, affermazioni diverse e contrastanti possono essere entrambe vere in quanto coerenti con sistemi diversi di credenze ${ }^{5}$.

Quale concezione risulta più adeguata in politica? Da un certo punto di vista, proprio l'intento di arginare la deriva della propaganda sembrerebbe reclamare una concezione corrispondentista della verità, da contrapporre alla postverità populista. D'altra parte, la concezione corrispondentista presenta il difetto di motivare un'insorgenza continua del dubbio scettico. Richiedere una corrispondenza tra le parole e le cose è un compito molto esigente, che non sempre può essere soddisfatto - perché le teorie cambiano, perché emergono nuove prove di cui prima non si conosceva l'esistenza ecc. La conseguenza è un'ansia continua riguardo la veridicità di certe affermazioni che, non potendo essere stabilizzate una volta per tutte, rendono strutturale la condizione di incertezza. Quest'ansia poi comporta la paralisi del momento decisorio: come si fa a scegliere tra corsi di azione differenti se non ci sono garanzie riguardo la fondatezza dei presupposti di ciascuno di esso?

La teoria coerentista, d'altra parte, richiede il radicamento delle proposizioni ritenute vere in un complesso di verità che definiscono, al contempo, i confini di una comunità. Ma in condizioni di radicale pluralismo è difficile pensare alla possibilità di individuare comunità così estese da rendere possibile un'autentica discussione delle tematiche di interesse pubblico. Il rischio, piuttosto, è quello della segmentazione del dibattito in tanti blocchi contrapposti e non comunicanti. Come conseguenza, il concetto di verità si abbassa a quello di verità entro un dato sistema, una data comunità. Pertanto, entrambe le concezioni, per diversi motivi, sembrano poco spendibili all'interno del dominio politico.

Per superare questa impasse vorrei far leva su una diversa intuizione. Il modo in cui il problema della verità entra nel circuito democratico è quello del dato che supporta la decisione politica. Ora, è evidente che richiedere una completa certezza è paralizzante, in quanto autorizza il continuo ripresentarsi del dubbio scettico riguardo alla verità dei fatti ipotizzati. Una proposta calibrata sulle specificità del discorso politico $\mathrm{e}$, in particolare, democratico è incompatibile con questa impostazione. Una concezione della verità più meditata dovrebbe articolare l'attitudine a rivedere le affermazioni che, di volta in volta, vengono avanzate. Le affermazioni ritenute vere sono sempre tali, fino a prova contraria. Ciò, da un lato, impedisce di attribuire eccessiva enfasi al problema, generalmente insolubile, della dimostrazione di corrispondenza alla realtà delle asserzioni; dall'altra, permette di focalizzarsi sui problemi realmente importanti della pratica democratica, superando le contrapposizioni settarie: chi sono i soggetti che parlano, qual è la loro competenza, quali 
regole procedurali sono state adottate, in che modo esse promettono di porci al riparo dal pericolo delle ingerenze da parte del potere.

Come può una simile concezione della verità disinnescare la deriva populista? La mossa cruciale è quella di ripristinare la distinzione di livelli tra «deliberazioni» e «deliberazioni corrette», non attraverso la definizione di un insieme di regole procedurali ma attraverso la proposizione di un insieme di principî sostanziali che orientino il contenuto delle deliberazioni stesse. Tali principî dovrebbero imporsi in quanto standard, essi stessi rivedibili, che guidano le deliberazioni sottostanti ${ }^{6}$. Non quindi il tentativo, per lo più infruttuoso, di smontare dal loro interno, accettandone pertanto il frame, le soluzioni dei populisti, ma la proposizione di concezioni, principî e valori alternativi a quelli veicolati dai populisti, che possano organizzare un sistema di scelte politiche difformi. Una democrazia perfettamente inclusiva è un traguardo inattingibile. La prospettiva di un progresso, sotto questo punto di vista, si lega alla possibilità di mobilitare idee e immagini che facciano presa sui destinatari del messaggio politico ${ }^{7}$.

${ }^{6}$ Il problema più delicato riguarda la precisazione delle modalità con le quali un insieme di principî sostanziali viene sostituito da un altro. In questa sede non è possibile approfondire la questione ma dovrebbe rimanere fermo che il passaggio assomiglia a un 'cambio di paradigma' nel senso di Thomas Kuhn - cfr. Th. S. Kuhn, La struttura delle rivoluzioni scientifiche (ed. or. 1970), Einaudi, Torino 1978 - come tale non governabile da criteri di razionalità trans-storici. Cfr. anche Marchettoni, Relativismo e differenza culturale, cit.

7 Cfr. Ferrara, The Democratic Horizon, cit., cap. 1. 


\section{Bibliografia}

Accarino B., Rappresentanza, il Mulino, Bologna 1999.

Ackerman B., Fishkin J.S., Deliberation Day, Yale University Press, New Haven 2004.

Adorno Th. W., Minima moralia. Meditazioni della vita offesa (ed. or. 1951), a cura di L. Ceppa, Einaudi, Torino 1994.

Alber J., Dalla carità allo Stato sociale (ed. or. 1982), il Mulino, Bologna 1986.

Allegretti U. (a cura di), Democrazia partecipativa. Esperienze e prospettive in Italia e in Europa, Firenze University Press, Firenze 2010.

Althusser L., Per Marx (ed. or. 1965), nota introduttiva di C. Luporini, Editori Riuniti, Roma 1974.

- , Lo Stato e i suoi apparati, a cura di R. Finelli, Editori Riuniti, Roma 1997. Anderson B., Comunità immaginate. Origini e fortuna dei nazionalismi (ed. or. 1983, 1991), prefazione di M. D’Eramo, Laterza, Roma-Bari 2018.

Annas J., An Introduction to Plato's Republic, Oxford University Press, Oxford 1981.

Anonimo Ateniese, La democrazia come violenza, a cura di L. Canfora, Sellerio, Palermo 1982.

Archibugi D., Cittadini del mondo. Verso una democrazia cosmopolitica, il Saggiatore, Milano 2009.

Aristotele, Politica, commento di T.J. Saunders, R. Robinson, D. Keyt e R. Kraut, 2 voll., Fondazione Lorenzo Valla-Mondadori, Milano 2014-2015.

-, Costituzione degli Ateniesi, a cura di P.J. Rhodes, Fondazione Lorenzo Valla-Mondadori, Milano 2016.

Ashcraft R., Revolutionary Politics and Locke's Two Treatises of Government, Princeton University Press, Princeton 1986.

Ascheri M., Le città-Stato. Le radici del municipalismo e del repubblicanesimo italiano, il Mulino, Bologna 2006.

Avineri Sh., La teoria hegeliana dello Stato (ed. or. 1972), Laterza, RomaBari 1973. 
-, Il pensiero politico e sociale di Karl Marx (ed. or. 1968), il Mulino, Bologna 1972.

Baccelli L., Critica del repubblicanesimo, Laterza, Roma-Bari 2003.

-, Political Imagination, Conflict, and Democracy: Machiavelli's Republican Realism, in D.C. Johnston, N. Urbinati, C. Vergara (eds.), Machiavelli on Liberty and Conflict, The University of Chicago Press, Chicago 2017.

Bader R., Robert Nozick, Bloomsbury, London 2013.

Balibar É., Spinoza e la politica (ed. or. 1990), Manifestolibri, Roma 1996.

-, Cittadinanza, Boringhieri, Torino 2012.

Barbera A., Fusaro C., Il governo delle democrazie, il Mulino, Bologna 2009. Barberis M., Benjamin Constant. Rivoluzione, costituzione, progresso, il Mulino, Bologna 1988.

Bailyn B., The Ideological Origins of the American Revolution, Harvard University Press, Cambridge, Mass. 1992.

Beetham D., La teoria politica di Max Weber (ed. or. 1985), il Mulino, Bologna 1989.

Belloni R., Moschella M., Sicurelli D. (a cura di), Le organizzazioni internazionali. Struttura, funzioni, impatto, il Mulino, Bologna 2013.

Benhabib S., Toward a Deliberative Model of Democratic Legitimacy, in Ead. (ed.), Democracy and Difference, Princeton University Press, Princeton 1996.

Bentham J., Un frammento sul governo (ed. or. 1766), a cura di S. Marcucci, Giuffrè, Milano 1990.

-, Introduzione ai principî della morale e della legislazione (ed. or. 1789), a cura di E. Lecaldano, Utet, Torino 1998.

Bien G., La filosofia politica di Aristotele (ed. or. 1973), il Mulino, Bologna 1985.

Black A., Political Thought in Europe 1250-1450, Cambridge University Press, Cambridge 1992.

Bobbio N., Il futuro della democrazia, Einaudi, Torino 1984.

-, Thomas Hobbes, Einaudi, Torino 1989.

-, L'età dei diritti, Einaudi, Torino 1990.

-, Stato, governo, società. Frammenti di un dizionario politico, Einaudi, Torino 1995.

Bobonich C., Plato's Utopia Recast: His Later Ethics and Politics, Oxford University Press, Oxford 2004.

Boeri T., Populismo e Stato sociale, Laterza, Roma-Bari 2017.

Bohman J., Public Deliberation: Pluralism, Complexity, and Democracy, Mit Press, Cambridge 1997.

Brennan J., Contro la democrazia (ed. or. 2016), Luiss University Press, Roma 2018.

Briguglia G., Marsilio da Padova, Carocci, Roma 2013.

Brink D., Mill's Progressive Principles, Clarendon Press, Oxford 2013.

Brooks Th., Hegel's Political Philosophy: A Systematic Reading of the Philosophy of Right, Edinburgh University Press, Edinburgh 2012. 
Brucker G.A., Dal Comune alla Signoria. La vita pubblica a Firenze nel primo Rinascimento (ed. or. 1977), il Mulino, Bologna 1981.

Buchanan J.M., Tullock G., The Calculus of Consent. Logical Foundations of Constitutional Democracy, University of Michigan Press, Ann Arbour 1962.

Calise M., Il partito personale. I due corpi del leader, Laterza, Roma-Bari, 2010.

-, La democrazia del leader, Laterza, Roma-Bari, 2016.

Cambiano G., Platone e le tecniche, Laterza, Roma-Bari 1991.

-, Polis. Un modello per la cultura europea, Laterza, Roma-Bari 2000.

-, Come nave in tempesta. Il governo della città in Platone e Aristotele, Laterza, Roma-Bari 2016.

Canfora L., La democrazia. Storia di un'ideologia, Laterza, Roma-Bari 2004.

-, Se la democrazia degli antichi sia ancora utile per emendare quella dei moderni, in C. Altini (a cura di), Democrazia. Storia e teoria di un'esperienza filosofica e politica, il Mulino, Bologna 2011.

-, Tucidide. La menzogna, la colpa, l'esilio, Laterza, Roma-Bari 2016.

Carillo G., Nel molto c'è il tutto, la democrazia nel dibattito sui regimi politici (Erodoto, III, 8o, 1-6), in G. Duso (a cura di), Oltre la democrazia. Un itinerario attraverso i classici, Carocci, Roma 2004.

Cartledge P., Democracy: A Life, Oxford University Press, Oxford 2016.

Carver T. (ed.), The Cambridge Companion to Marx, Cambridge University Press, Cambridge 2008.

Casadei Th., I diritti sociali. Un percorso filosofico-giuridico, Firenze University Press, Firenze 2012.

Casalini B., Nei limiti del compasso: Locke e le origini della cultura politica e costituzionale americana, Mimesis, Milano-Udine 2002.

Cassese S., Il diritto globale. Giustizia e democrazia oltre lo Stato, Einaudi, Torino 2009.

-, La democrazia e i suoi limiti, Mondadori, Milano 2017.

Cerutti F., Sfide globali per il leviatano. Una filosofia politica delle armi nucleari e del riscaldamento globale, Vita \& Pensiero, Milano 2009.

Cesa C., Hegel filosofo politico, Guida, Napoli 1976.

Cesarale G., La mediazione che sparisce. La società civile in Hegel, Carocci, Roma 2009.

Chignola S., Il tempo rovesciato. La Restaurazione e il governo della democrazia, il Mulino, Bologna 2011.

Clifford J., I frutti puri impazziscono. Etnografia, letteratura e arte nel secolo XX (ed. or. 1988), Boringhieri, Torino 1999.

Cohen J., Procedure and Substance in Deliberative Democracy, in S. Benhabib (ed.), Democracy and Difference, Princeton University Press, Princeton 1996.

-, Rousseau: A Free Community of Equals, Oxford University Press, Oxford 2010.

Coleman J., A History of Political Thought: From the Middle Ages to the Renaissance, Blackwell, Oxford 2000. 
Conetti M., Il pensiero politico dei giuristi, in M. Fumagalli Beonio Brocchieri et al., Il pensiero politico medievale, Laterza, Roma-Bari 2000.

Consorti P., Diritto e religione, Laterza, Roma-Bari 2014.

Constant B., Principî di politica (ed. or. 1815), a cura di di U. Cerroni, Editori Riuniti, Roma 1970.

-, La libertà degli antichi, paragonata a quella dei moderni (ed. or. 1819), a cura di G. Paoletti, Einaudi, Torino 2005.

Cosenza G., Semiotica e comunicazione politica, Laterza, Roma-Bari 2018.

Costa P., Civitas. Storia della cittadinanza in Europa, vol. 1, Dalla civiltà comunale al Settecento, Laterza, Roma-Bari 1999.

-, Civitas. Storia della cittadinanza in Europa, vol. 2, L'età delle rivoluzioni, Laterza, Roma-Bari 2000.

-, Civitas. Storia della cittadinanza in Europa, vol. 3, La civiltà liberale, Laterza, Roma-Bari 2001.

-, Cittadinanza, Laterza, Roma-Bari 2005.

Crozier M., Huntington S.P., Watanuki J., La crisi della democrazia. Rapporto sulla governabilità delle democrazie alla Commissione trilaterale (ed. or. 1975), FrancoAngeli, Milano 1977.

D'Andrea D., Prometeo e Ulisse. Natura umana e ordine politico in Thomas Hobbes, La Nuova Italia Scientifica, Roma 1997.

-, L’incubo degli ultimi uomini. Etica e politica in Max Weber, Carocci, Roma 2005.

Dahl R., La democrazia e i suoi critici (ed. or. 1989), Editori Riuniti, Roma 1990.

Dal Lago A., Populismo digitale. La crisi, la rete e la nuova destra, Raffaello Cortina, Milano 2017.

De Benedictis A., Politica, governo e istituzioni nell'Europa moderna, il Mulino, Bologna 2001.

De Giovanni B., Alle origini della democrazia di massa, Editoriale Scientifica, Napoli 2013.

De Pascale C., Etica e diritto. La filosofia pratica di Fichte e le sue ascendenze kantiane, il Mulino, Bologna 1995.

De Sanctis F.M., Tocqueville: democrazia e rivoluzione, Editoriale Scientifica, Napoli 2000.

Del Lucchese F., Tumulti e indignatio. Conflitto, diritto e moltitudine in Machiavelli e Spinoza, Ghibli, Milano 2004.

della Porta D., I partiti politici, il Mulino, Bologna 2015.

della Porta D., Diani M., I movimenti sociali, La Nuova Italia Scientifica, Roma 1997.

Den Uyl D.J., Power, State and Freedom: An interpretation of Spinoza's Political Philosophy, Van Gorcum \& Company, Assen 1983.

Dent N.J.H., Rousseau, Routledge, London 2005.

Dichiarazione dei Diritti dell'Uomo e del Cittadino (ed. or. 1789), introduzione di T.E. Frosini, Liberilibri, Macerata 2008.

Downs A., Teoria economica della democrazia (ed. or. 1957), il Mulino, Bologna 1989. 
Dryzek J.S., Deliberative Democracy and Beyond: Liberals, Critics, Contestations, Oxford University Press, Oxford 2000.

-, Foundations and Frontiers of Deliberative Governance, Oxford University Press, Oxford 2010.

Dunn J., Il pensiero politico di Locke (ed. or. 1979), il Mulino, Bologna 1992. Duso G., La rappresentanza politica. Genesi e crisi del concetto, FrancoAngeli, Milano 2003.

Elster J., The Market and the Forum: Three Varieties of Political Theory, in J. Bohman, W. Rehg (eds.), Deliberative Democracy, Mit Press, Cambridge 1997.

Facchi A., I diritti nell'Europa multiculturale. Pluralismo normativo e immigrazione, Laterza, Roma-Bari 2001.

-, Breve storia dei diritti umani. Dai diritti dell'uomo ai diritti delle donne, il Mulino, Bologna 2013.

Ferrajoli L., La democrazia attraverso i diritti, Laterza, Roma-Bari 2013.

Ferrara A., Modernità e autenticità. Saggio sul pensiero sociale ed etico di J.J. Rousseau, Armando, Roma 1989.

- (a cura di), Comunitarismo e liberalismo, Editori Riuniti, Roma 2000.

-, The Democratic Horizon, Cambridge University Press, New York 2014.

Ferraris M., Postverità e altri enigmi, il Mulino, Bologna 2017.

Ferrera M., Modelli di solidarietà. Politica e riforme sociali nelle democrazie, il Mulino, Bologna 1993.

Fichte J.G., Contributo per rettificare i giudizi del pubblico sulla rivoluzione francese (ed. or. 1793), in Id., Sulla rivoluzione francese, a cura di V.E. Alfieri, Laterza, Roma-Bari 1974.

-, Fondamenti del diritto naturale (ed. or. 1796), a cura di L. Fonnesu, Laterza, Roma-Bari 1994.

-, Discorsi alla nazione tedesca (ed. or. 1807-1808), a cura di G. Rametta, Laterza, Roma-Bari 2003.

Finley M.I., La democrazia degli antichi e dei moderni (ed. or. 1985), Laterza, Roma-Bari 2010.

Finnis J., Aquinas: Moral, Political, and Legal Theory, Oxford University Press, Oxford 1998.

Fioravanti M. (a cura di), Lo Stato moderno in Europa. Istituzioni e diritto, Laterza, Roma-Bari 2017.

Fishkin J.S., Democracy and Deliberation: New Directions for Democratic Reforms, Yale University Press, New Haven 1991.

-, When the People Speak: Deliberative Democracy and Public Consultation, Oxford University Press, Oxford 2009.

-, Democracy When the People Are Thinking: Revitalizing Our Politics Through Public Deliberation, Oxford University Press, Oxford 2018.

Floridia A., Un’idea deliberativa della democrazia. Genealogia e principî, il Mulino, Bologna 2017.

Foucault M., Storia della follia nell'età classica (ed. or. 1961), Bur, Milano 2011. 
-, Nascita della clinica (ed. or. 1963), Einaudi, Torino 1965.

-, Sorvegliare e punire (ed. or. 1975), Einaudi, Torino 1976.

-, «Bisogna difendere la società» (ed. or. 1997), a cura di M. Bertani e A. Fontana, Feltrinelli, Milano 1998.

-, Nascita della biopolitica (ed. or. 2004), a cura di M. Senellart, Feltrinelli, Milano 2005.

Freeman S., Rawls, Routledge, London 2007.

Gabrieli V. (a cura di), Puritanesimo e libertà. Dibattiti e libelli, Einaudi, Torino 1956.

Gallino L., Il colpo di Stato di banche e governi. L'attacco alla democrazia in Europa, Einaudi, Torino 2013.

Gellner E., Nazioni e nazionalismo (ed. or. 1983), Editori Riuniti, Roma 1992.

Giorgi C., Le istituzioni del welfare, in M. Meriggi, L. Tedoldi (a cura di), Storia delle istituzioni politiche. Dall'antico regime all'era globale, Carocci, Roma 2014.

Goldoni M., La dottrina costituzionale di Sieyès, Firenze University Press, Firenze 2009.

Gometz G., Democrazia elettronica. Teoria e tecniche, Ets, Pisa 2017.

Gonnelli F., La filosofia politica di Kant, Laterza, Roma-Bari 1996.

Gutman A., Rawls on the Relationship between Liberalism and Democracy, in S. Freeman (ed.), The Cambridge Companion to Rawls, Cambridge, University Press, Cambridge 2003.

Gutman A., Thompson D., Democracy and Disagreement, Harvard University Press, Cambridge 1997.

Habermas J., Storia e critica dell'opinione pubblica (ed. or. 1962, 1990), a cura di M. Carpitella, Laterza, Roma-Bari, 2002.

-, Teoria dell'agire comunicativo (ed. or. 1981), 2 voll., a cura di G.E. Rusconi, il Mulino, Bologna 1986.

-, Fatti e norme. Contributi a una teoria discorsiva del diritto e della democrazia (ed. or. 1992), a cura di L. Ceppa, Guerini e Associati, Milano 1996.

Hamilton A., Jay J., Madison J., The Federalist Papers (ed. or. 1788), trad. it. Il Federalista, a cura di G. Sacerdoti Mariani, Giappichelli, Torino 1997.

Hampton J., Hobbes and the Social Contract Tradition, Cambridge University Press, Cambridge 1986.

Hansen M.H., The Athenian Democracy in the Age of Demosthenes, Duckworth, London 1999.

-, Graphe paranomon. La sovranità del tribunale popolare ad Atene nel IV secolo a.C. e l'azione pubblica contro proposte incostituzionali (ed. or. 1991), a cura di M.C. Rogozinski, Giappichelli, Torino 2001.

Harris E.M., Democracy and the Rule of Law in Classical Athens: Essays on Law, Society, and Politics, Cambridge University Press, Cambridge 2006.

Hayek F.A. (von), La via della schiavitù (ed. or. 1944, 1956), a cura di D. Antiseri e R. De Mucci, Rusconi, Milano 1995. 
-, Legge, legislazione e libertà. Critica dell'economia pianificata (ed. or. 1973-1979), a cura di A. Petroni e S. Monti Bragadin, il Saggiatore, Milano 1994.

Hegel G.W.F., Le maniere scientifiche di trattare il diritto naturale (ed. or. 1803), a cura di C. Sabbatini, Bompiani, Milano 2016.

-, Fenomenologia dello spirito (ed. or. 1807), a cura. di V. Cicero, Rusconi, Milano 1995.

-, Lineamenti di filosofia del diritto (ed. or. 1821), a cura di G. Marini, Laterza, Roma-Bari 1987.

Hill Ch., The World Turned Upside Down: Radical Ideas During the English Revolution, Penguin, London 1991.

-, The Century of Revolution, 1603-1714, Routledge, Abingdon 2001.

Hobbes Th., Elementi di legge naturale e politica (ed. or. 1640), a cura di A. Pacchi, La Nuova Italia, Firenze 1968.

-, De cive. Elementi filosofici sul cittadino (ed. or. 1642), a cura di T. Magri, Editori Riuniti, Roma 1979.

-, Leviatano (ed. or. 1651), a cura di R. Santi, Bompiani, Milano 2001.

Hoekstra K., A Lion in the House: Hobbes and Democracy, in A. Brett, J. Tully, H. Hamilton-Bleakley (eds.), Rethinking the Foundations of Modern Political Thought, Cambridge University Press, Cambridge 2006.

Hofmann H., Rappresentanza-rappresentazione. Parola e concetto dall'antichità all'Ottocento (ed. or. 1974), Giuffrè, Milano 2007.

Honneth A., Il diritto della libertà. Lineamenti per un'eticità democratica (ed. or. 2011), Codice, Torino 2015.

Horkheimer M., Filosofia e teoria critica, a cura di S. Petrucciani, Einaudi, Torino 2003.

Horkheimer M., Adorno Th.W., Dialettica dell'illuminismo (ed. or. 1944), introduzione di C. Galli, Einaudi, Torino 1997.

Iofrida M., Melegari D., Foucault, Carocci, Roma 2017.

Israel J., Radical Enlightenment: Philosophy and the Making of Modernity 1650-1750, Oxford University Press, Oxford 2002.

-, Enlightenment Contested, Enlightenment Contested: Philosophy, Modernity, and the Emancipation of Man 1670-1752, Oxford University Press, Oxford 2008.

-, Una rivoluzione della mente. L'illuminismo radicale e le origini intellettuali della democrazia moderna (ed. or. 2009), Einaudi, Torino 2011.

-, Democratic Enlightenment: Philosophy, Revolution, and Human Rights 1750-179o, Oxford University Press, Oxford 2013.

-, La Rivoluzione francese. Una storia intellettuale dai Diritti dell'uomo a Robespierre (ed. or. 2015), Einaudi, Torino 2016.

Jaume L., Tocqueville. Les sources aristocratiques de la liberté, Fayard, Paris 2008. 
Kant I., Sopra il detto comune: "questo può essere giusto in teoria ma non vale per la pratica» (ed. or. 1793), in Id., Scritti politici e filosofia della storia e del diritto, a cura di G. Solari et al., Utet, Torino 1956.

-, Per la pace perpetua (ed. or. 1795), in Id., Scritti politici e filosofia della storia e del diritto, a cura di G. Solari et al., Utet, Torino 1956

-, Metafisica dei costumi (ed. or. 1797), a cura di G. Landolfi Petrone, Bompiani, Milano 2006.

Kavka G., Hobbesian Moral and Political Theory, Princeton University Press, Princeton 1986.

Kazin M., The Populist Persuasion: An American History, Basic Books, New York 1998.

Kelsen H., Teoria generale del diritto e dello Stato (ed. or. 1961), a cura di S. Cotta e G. Treves, Edizioni di Comunità, Milano 1963.

-, La giustizia costituzionale, a cura di C. Geraci, Giuffrè, Milano 1981.

-, La democrazia, a cura di M. Barberis, il Mulino, Bologna 1984.

Keynes J.M., Teoria generale dell'occupazione, dell'interesse e della moneta (ed. or. 1936), a cura di T. Cozzi, Utet, Torino 2006.

Kersting W., Wohlgeordnete Freiheit. Immanuel Kants Rechts- und Staatsphilosophie, de Gruyter, Berlin 1984.

Kirchheimer O., La trasformazione dei sistemi partitici dell'Europa Occidentale, in G. Sivini (a cura di), Sociologia dei partiti politici, il Mulino, Bologna 1979.

Klosko G., The Development of Plato's Political Theory, Oxford University Press, Oxford 2006.

Kraut R., Aristotle: Political Philosophy, Oxford University Press, Oxford 2002.

Kuhn Th. S., La struttura delle rivoluzioni scientifiche (ed. or. 1970), Einaudi, Torino 1978.

La Costituzione degli Stati Uniti. Storia, testo inglese, nuova traduzione, commento e note, a cura di F. Tonello, Mondadori, Milano 2010.

La Dichiarazione d'indipendenza degli Stati Uniti d'America, a cura di T. Bonazzi, Marsilio, Venezia 2001.

Laclau E., La ragione populista (ed. or. 2005), Laterza, Roma-Bari 2008.

Laudani R., Politica come movimento. Il pensiero di Herbert Marcuse, il Mulino, Bologna 2005.

Lintott A., The Constitution of the Roman Republic, Oxford University Press, Oxford 2003.

Lipset S.M., Rokkan S., Strutture di clevage, sistemi partitici e allineamenti dell'elettorato, in S.M. Lipset, Istituzioni, partiti, società civile, a cura di L. Morlino, il Mulino, Bologna 2009.

Locke J., Saggi sulla legge di natura, Laterza, Roma-Bari 1973.

-, Due trattati sul governo e altri scritti politici (e il Patriarca di Robert Filmer), a cura di L. Pareyson, Utet, Torino 1982.

Lorusso A.M., Postverità, Laterza, Roma-Bari 2018.

Loughlin M., Foundations of Public Law, Oxford University Press, Oxford 2010. 
Machiavelli N., Opere, a cura di R. Rinaldi, 2 voll., Utet, Torino 1999.

MacIlwain C.H., Costituzionalismo antico e moderno (ed. or. 1947), il Mulino, Bologna 1990.

MacIntyre A., Dopo la virtù. Saggio di teoria morale (ed. or. 1981), Feltrinelli, Milano 1988.

Mack E., Libertarianism, Polity Press, Cambridge 2018.

Macpherson C.B., Libertà e proprietà alle origini della società borghese (ed. or. 1962), Isedi, Milano 1973.

-, La vita e i tempi della democrazia liberale (ed. or. 1977), il Saggiatore, Milano 1980.

Maffettone S., Introduzione a Rawls, Laterza, Roma-Bari 2010.

Manin B., On Legitimacy and Political Deliberation, «Political Theory», 15 (1987), pp. 338-368.

-, Principî del governo rappresentativo (ed. or. 1997), il Mulino, Bologna 2010.

Mannoni S., Une et indivisible. Storia dell'accentramento amministrativo in Francia, vol. I, La formazione del sistema (1661-1815), Giuffrè, Milano 1994.

Mannori L., Le istituzioni politiche dell'antico regime, in M. Meriggi, L. Tedoldi (a cura di), Storia delle istituzioni politiche. Dall'antico regime all'era globale, Carocci, Roma 2014.

Mannori L., Sordi B., Storia del diritto amministrativo, Laterza, Roma-Bari 2013.

Marchettoni L., Ockham e l'origine dei diritti soggettivi, «Quaderni fiorentini per la storia del pensiero giuridico moderno», 37, 2008, pp. 19-64.

-, I diritti umani tra universalismo e particolarismo, Giappichelli, Torino 2012.

-, Relativismo e differenza culturale, Nuova Cultura, Roma 2012.

-, Teorie del populismo, «Jura Gentium», XIV (2), 2017, pp. 161-171.

Marcuse H., Eros e civiltà (ed. or. 1955), Einaudi, Torino 1967.

-, L'uomo a una dimensione. L'ideologia di una società industriale avanzata (ed. or. 1964), Einaudi, Torino 1967.

-, Ragione e rivoluzione. Hegel e il sorgere della teoria sociale (ed. or. 1941), introduzione di C. Galli, il Mulino, Bologna 1998.

Marshall Th. H., Cittadinanza e classe sociale (ed. or. 1963), a cura di P. Maranini, Utet, Torino 1976.

Marsilio da Padova, Il difensore della pace, a cura di C. Vasoli, Utet, Torino 1960.

Martel J., Subverting the Leviathan: Reading Thomas Hobbes as a Radical Democrat, Columbia University Press, New York 2007.

Martinich A.P., Hobbes, Routledge, Abingdon 2005.

Marx K., Critica della filosofia hegeliana del diritto pubblico (ed. or. 1844), a cura di M. Prospero, Editori Riuniti, Roma 2016.

-, Sulla questione ebraica (ed. or. 1844), a cura di D. Fusaro, Bompiani, Milano 2007.

-, La guerra civile in Francia (ed. or. 1871), a cura di P. Togliatti, Editori Riuniti, Roma 1974. 
Marx K., Engels F., Il manifesto del partito comunista (ed. or. 1847), a cura di B. Bongiovanni, Einaudi, Torino 2014.

Marzocchi V., Per un'etica pubblica. Giustificare la democrazia, Liguori, Napoli 2000.

Matheron A., Individu et communauté chez Spinoza, Les Editions de Minuit, Paris 1969.

Matteucci N., La rivoluzione americana: una rivoluzione costituzionale, il Mulino, Bologna 1987.

-, Lo Stato moderno, il Mulino, Bologna 1993.

Mazzoleni G., La comunicazione politica, il Mulino, Bologna 2012.

McCormick J.P., Weber, Habermas and Transformations of the European State: Constitutional, Social, and Supranational Democracy, Cambridge University Press, Cambridge 2007.

-, Machiavellian Democracy, Cambridge University Press, Cambridge 2011.

Meniconi A., Le istituzioni politiche del totalitarismo: Italia, Germania, Unione Sovietica, in M. Meriggi, L. Tedoldi (a cura di), Storia delle istituzioni politiche. Dall'antico regime all'era globale, Carocci, Roma 2014.

Meriggi M., Dal liberalismo alla democrazia (1848-1914), in M. Meriggi, L. Tedoldi (a cura di), Storia delle istituzioni politiche. Dall'antico regime all'era globale, Carocci, Roma 2014.

Merlo M., Marsilio da Padova. La politica come grammatica del mutamento, FrancoAngeli, Milano 2003.

Michels R., La sociologia del partito politico nella democrazia moderna (ed. or. 1911), il Mulino, Bologna 1966.

Mill J.S., Sulla libertà (ed. or. 1859), a cura di G. Mollica, Bompiani, Milano 2000.

-, Considerazioni sul governo rappresentativo (ed. or. 1861), a cura di M. Prospero, Editori Riuniti, Roma 1997.

-, L’America e la democrazia, a cura di P. Adamo, Bompiani, Milano 2005.

Miller D., J.S. Mill: Moral, Social, and Political Thought, Polity Press, Cambridge 2010.

Miller F.D., Jr., Nature, Justice, and Rights in Aristotle's Politics, Oxford University Press, Oxford 1995.

Moffitt B., The Global Rise of Populism: Performance, Political Style, and Representation, Stanford University Press, Stanford 2016.

Mommsen W.J., Max Weber e la politica tedesca (ed. or. 1974), il Mulino, Bologna 1993.

Montesquieu, Lo spirito delle leggi (ed. or. 1748), in Id., Tutte le opere [17211754], a cura di D. Felice, Bompiani, Milano 2014.

Mosca G., Sulla teorica dei governi e sul governo parlamentare (ed. or. 1884), in Id., Scritti politici, 2 voll., a cura di G. Sola, Utet, Torino 1982.

-, Elementi di scienza politica (ed. or. 1895, 1922), in Id., Scritti politici, 2 voll., a cura di G. Sola, Utet, Torino 1982.

Mossé C., Pericle. L’inventore della democrazia (ed. or. 2005), Laterza, Roma-Bari 2006. 
Mounk Y., Popolo vs democrazia. Dalla cittadinanza alla dittatura elettorale (ed. or. 2018), Feltrinelli, Milano 2018.

Mudde C., Rovira Kaltwasser C., Populism: A Very Short Introduction, Oxford University Press, Oxford 2017.

Müller J.-W., Cos'è il populismo? (ed. or. 2016), Università Bocconi Editore, Milano 2017.

Musti D., Demokratía. Origini di un’idea, Laterza, Roma-Bari 1995.

Negri A., Spinoza. L’anomalia selvaggia. Spinoza sovversivo. Democrazia ed eternità in Spinoza, Deriveapprodi, Roma 1998.

Nida-Rümelin J., Democrazia e verità (ed. or. 2006), FrancoAngeli, Milano 2015 .

Novarese D., Le istituzioni politiche della Restaurazione, in M. Meriggi, L. Tedoldi (a cura di), Storia delle istituzioni politiche. Dall'antico regime all'era globale, Carocci, Roma 2014.

Nozick R., Anarchia, Stato e utopia (ed. or. 1974), presentazione di S. Maffettone, il Saggiatore, Milano 2008.

Ober J., Political Dissent in Demochratic Athens: Intellectual Critics of Popular Rule, Princeton University Press, Princeton 1998.

Omaggio V., Marsilio da Padova. Diritto e politica nel Defensor Pacis, Editoriale Scientifica, Napoli 1995.

Osborne R., Athens and Athenian Democracy, Cambridge University Press, Cambridge 2014.

Pacchiani C., Democrazia e costituzione. La lezione di Aristotele, in G. Duso (a cura di), Oltre la democrazia. Un itinerario attraverso i classici, Carocci, Roma 2004.

Palombella G., Ragione e immaginazione. Herbert Marcuse 1928-1955, De Donato, Bari 1982.

-, Costituzione e sovranità. Il senso della democrazia costituzionale, Dedalo, Bari 1997.

-, Dopo la certezza. Il diritto in equilibrio tra giustizia e democrazia, Dedalo, Bari 2006.

-, È possibile una legalità globale? Il Rule of law e la governance del mondo, il Mulino, Bologna 2012.

Panebianco A., Modelli di partito, il Mulino, Bologna 1982.

Pareto V., Trattato di sociologia generale (ed. or. 1916), a cura di G. Busino, Utet, Torino 1988.

Pariotti E., I diritti umani. Concetto, teoria, evoluzione, Cedam, Milano 2013.

Parry G., Le élites politiche (ed. or. 1969), il Mulino, Bologna 1972.

Pateman C., Participation and Democratic Theory, Cambridge University Press, Cambridge 1970.

Petrucciani S., Introduzione ad Habermas, Laterza, Roma-Bari 2000.

-, Marx, Carocci, Roma 2009.

-, Democrazia, Einaudi, Torino 2014. 
-, A lezione da Adorno. Filosofia, società, estetica, Manifestolibri, Roma 2017.

Pietropaoli S., Schmitt, Carocci, Roma 2012.

Piketty Th., Il capitale nel XXI secolo (ed. or. 2013), Bompiani, Milano 2014.

Pitkin H.F., Il concetto di rappresentanza (ed. or. 1967), Rubbettino, Soveria Mannelli 2017.

Platone, Politico, a cura di M. Migliori, Bompiani, Milano 2001.

-, Leggi, a cura di F. Ferrari, Bur, Milano 2005.

-, La Repubblica, a cura di M. Vegetti, Bur, Milano 2007.

Pocock J.G.A., Il momento machiavelliano. Il pensiero politico fiorentino e la tradizione repubblicana anglosassone (ed. or. 1975), il Mulino, Bologna 1980.

Polsi A., Storia dell'ONU, Laterza, Roma-Bari 2009.

Portinaro P.P., Stato, il Mulino, Bologna 1999.

Preterossi G., I luoghi della politica. Figure istituzionali della filosofia del diritto hegeliana, Guerini e Associati, Milano 1992.

-, Carl Schmitt e la tradizione moderna, Laterza, Roma-Bari 1996.

-, Ciò che resta della democrazia, Laterza, Roma-Bari 2015.

Pulcini E., L’individuo senza passioni. Individualismo moderno e perdita del legame sociale, Boringhieri, Torino 2001.

Quattrociocchi W., Vicini A., Liberi di crederci. Informazione, internet e post-verità, Codice, Torino 2018.

Rametta G., Politica e democrazia nell'idealismo tedesco, in G. Duso (a cura di), Oltre la democrazia. Un itinerario attraverso i classici, Carocci, Roma 2004.

Rawls J., Una teoria della giustizia (ed. or. 1971, 1999), a cura di S. Maffettone, Feltrinelli, Milano 2008.

-, Liberalismo politico (ed. or. 1993, 1996, 2005), a cura di S. Veca, Einaudi, Torino 2012.

Re L., Il liberalismo coloniale di Alexis de Tocqueville, Giappichelli, Torino 2012.

Revelli M., Populismo 2.o, Einaudi, Torino 2017.

Rials S., La déclaration des droits de l'homme et du citoyen, Hachette, Paris 1988.

Riesbeck D.J., Aristotle on Political Community, Cambridge University Press, Cambridge 2016.

Ripstein A., Force and Freedom: Kant's Legal and Political Philosophy, Harvard University Press, Cambridge, Mass. 2009.

Roberts J.T., Athens on Trial: The Antidemocratic Tradition in Western Thought, Princeton University Press, Princeton 1994.

Robespierre M., La Rivoluzione giacobina, a cura di U. Cerroni, Editori Riuniti, Roma 1967.

Rosenfeld M., Arato A. (eds.), Habermas on Law and Democracy, University of California Press, Berkeley 1998. 
Rousseau J.J., Scritti politici, 3 voll., a cura di M. Garin, Laterza, Roma-Bari 1971.

Rubinstein N., Studies in Italian History in the Middle Age and Renaissance, vol. I, Political Thought and the Language of Politics, Edizioni di Storia e Letteratura, Roma 2004.

Runciman D., Hobbes's Theory of Representation: Anti-democratic or Protodemocratic?, in I. Shapiro et al., Political Representation, Cambridge University Press, Cambridge 2009.

Rush F. (ed.), The Cambridge Companion to Critical Theory, Cambridge University Pess, Cambridge 2008.

Samaras Th., Plato on Democracy, Peter Lang, New York 2002.

Sandel M.J., Il liberalismo e i limiti della giustizia (ed. or. 1982), Feltrinelli, Milano 1994.

Santaniello R., Capire l'Unione europea. Politiche, diritto, economia, il Mulino, Bologna 2016.

Santoro E., Autonomia individuale, libertà e diritti. Una critica dell'antropologia liberale, Ets, Pisa 1999.

Sartori G., The Theory of Democracy Revisited, 2 voll., Chatham House Publishers, Chatham 1987.

Schmitt C., Dottrina della Costituzione (ed. or. 1928), a cura di A. Caracciolo, Giuffrè, Milano 1984.

Schofield M., Plato: Political Philosophy, Oxford University Press, Oxford 2006.

Schofield Ph., Utility and Democracy: The Political Thought of Jeremy Bentham, Oxford University Press, New York 2006.

Schumpeter J.A., Capitalismo, socialismo, democrazia (ed. or. 1942), Etas, Milano 1994.

Scluchter W., Il paradosso della razionalizzazione. Studi su Max Weber (ed. or. 1980), Liguori, Napoli 1987.

Sharp A. (ed.), The English Levellers, Cambridge University Press, Cambridge 2008.

Shklar J., Montesquieu (ed. or. 1989), il Mulino, Bologna 1990.

Skinner Q., Le origini del pensiero politico moderno (ed. or. 1978), 2 voll., il Mulino, Bologna 1989.

-, Machiavelli (ed. or. 1981), il Mulino, Bologna 1999.

Sieyès E.-J., Opere e testimonianze politiche, a cura di G. Troisi Spagnolo, Giuffrè, Milano 1993.

Sintomer Y., Il potere al popolo. Giurie cittadine, sorteggio e democrazia partecipativa (ed. or. 2007), Dedalo, Bari 2009.

Sommerville J., The Social Contract (Contract of Government), in G. Klosko (ed.), The Oxford Handbook of History of Political Philosophy, Oxford University Press, Oxford 2011.

Spinoza B., Trattato teologico-politico (ed. or. 1670), in Id., Tutte le opere, a cura di A. Sangiacomo, Bompiani, Milano 2010.

-, Trattato politico, (ed. or. 1677), in Id., Tutte le opere, a cura di A. Sangiacomo, Bompiani, Milano 2010. 
Sreenivasan G., The Limits of Lockean Rights in Property, Oxford University Press, Oxford 1995.

Stockton D., The Classic Athenian Democracy, Oxford University Press, Oxford 1990.

Stolleis M., Geschichte des öffentlichen Rechts in Deutschland, vol. 3, Staatsund Verwaltungsrechtswissenschaft in Republik und Diktatur 1914-1945, C.H. Beck, München 1999.

Sunstein C.R., A cosa servono le Costituzioni. Dissenso politico e democrazia deliberativa (ed. or. 2001), il Mulino, Bologna 2009.

-, \#Republic. La democrazia nell'epoca dei social media, il Mulino, Bologna 2017.

Taylor A., Rivoluzioni americane. Una storia continentale, 1750-1804 (ed. or. 2017), Einaudi, Torino 2017.

Taylor Ch., Radici dell'io. La costruzione dell'identità moderna (ed. or. 1989), Feltrinelli 1993.

Taylor L.R., Roman Voting Assemblies: From the Hannibalic War to the Dictatorship of Caesar, The University of Michigan Press, Ann Arbour 1966.

Tedesco F., Hayek, Laterza, Roma-Bari 2005.

The Bill of Rights, introduzione di E. Wicks, Liberilibri, Macerata 2010.

Thorley J., Athenian Democracy, Routledge, London-New York 2004.

Tocqueville A. (de), Scritti politici, vol. I, a cura di N. Matteucci, Utet, Torino 1969.

-, La democrazia in America (ed. or. 1835-1840), a cura di M. Tesini, 2 voll., Città Aperta, Troina 2005.

-, Il pauperismo, a cura di M. Tesini, Edizioni Lavoro, Roma 1998.

-, Scritti penitenziari, a cura di L. Re, Edizioni di Storia e Letteratura, Roma 2002.

Tuccari F., Carisma e leadership nel pensiero di Max Weber, FrancoAngeli, Milano 1991.

Tuck R., Hobbes (ed. or. 1989), il Mulino, Bologna 2001.

-, Hobbes and Democracy, in A. Brett, J. Tully, H. Hamilton-Bleakley (eds.), Rethinking the Foundations of Modern Political Thought, Cambridge University Press, Cambridge 2006.

Tuckness A., Locke and the Legislative Point of View: Toleration, Contested Principles, and Law, Princeton University Press, Princeton 2002.

Tully J., A Discourse on Property. John Locke and His Adversaries, Cambridge University Press, Cambridge 1980.

-, Rediscovering America: The Two Treatises and Aboriginal Rights, in Id., An Approach to Political Philosophy: Locke in Contexts, Cambridge University Press, Cambridge 1993.

-, The Unfreedom of the Moderns in Comparison to their Ideals of Constitutional Democracy, in Id., Public Philosophy in a New Key, vol. 2: Imperialism and Civic Freedom, Cambridge University Press, Cambridge 2008. 
Ullmann W., Il pensiero politico nel Medioevo (ed. or. 1965), Laterza, RomaBari 1989.

Urbinati N., Representative Democracy: Principles and Genealogy, The University of Chicago Press, Chicago 2006.

-, Democrazia sfigurata. Il popolo tra opinione e verità (ed. or. 2014), Università Bocconi Editore, Milano 2017.

Urbinati N., Zakaras A. (eds.), J.S. Mill's Political Thought: A Bicentennial Reassessment, Cambridge University Press, Cambridge 2007.

Vegetti M., Quindici lezioni su Platone, Einaudi, Torino 2003.

Vertovec S., Super-diversity and its implications, «Ethnic and Racial Studies», XXX (2007), 6, pp. 1024-1054.

Volpe G., La verità, Carocci, Roma 2012.

Waldron J., God, Locke, and Equality: Christian Foundations in Locke's Political Thought, Cambridge University Press, Cambridge 2002.

Waley D., Dean T., The Italian City Republics, Routledge, Abingdon 2009.

Walzer M., La rivoluzione dei santi. Il puritanesimo alle origini del radicalismo politico (ed. or. 1982), Claudiana, Torino 1996.

Weber M., Dalla terra alla fabbrica. Scritti sui lavoratori agricoli e lo Stato nazionale (1892-1897), a cura di F. Ferraresi e S. Mezzadra, Laterza, Roma-Bari 2005.

-, L'etica protestante e lo spirito del capitalismo (ed. or. 1904-1905), in Id., Sociologia delle religioni, 2 voll., a cura di C. Sebastiani, Utet, Torino 1976.

-, Sistema elettorale e democrazia in Germania (ed. or. 1917), in Id., Scritti politici, introduzione di A. Bolaffi, trad. di A. Cariolato e E. Fongaro, Donzelli, Roma 1998.

-, Parlamento e governo (ed. or. 1918), a cura di F. Fusillo, Laterza, Roma-Bari 1982.

-, La scienza come professione (ed. or. 1919), a cura di P. Volonté, Rusconi, Milano 1997.

-, Il Presidente del Reich (ed. or. 1919), in Id., Scritti politici, introduzione di A. Bolaffi, trad. di A. Cariolato e E. Fongaro, Donzelli, Roma 1998.

-, La politica come professione (ed. or. 1919), in Id., Scritti politici, introduzione di A. Bolaffi, trad. di A. Cariolato e E. Fongaro, Donzelli, Roma 1998.

-, Economia e società. Dominio. L'economia in rapporto agli ordinamenti e alle forze sociali, a cura di M. Palma, Donzelli, Roma 2012.

Welch Ch., de Tocqueville, Oxford University Press, Oxford 1999.

White S. (ed.), The Cambridge Companion to Habermas, Cambridge University Press, Cambridge 1995.

Winstanley G., The Law of Freedom and other Writings (ed. or. 1652), Cambridge University Press, Cambridge 2008.

Wokler R., Rousseau (ed. or. 1995), il Mulino, Bologna 2001.

Wood A., Karl Marx, Routledge, London 2004. 
Wotton D., Leveller Democracy and the Puritan Revolution, in J.H. Burns, M. Goldie (eds.), Cambridge History of Political Thought, 1450-170o, Cambridge University Press, Cambridge 1991.

Young I.M., Le politiche della differenza (ed. or. 1990), Feltrinelli, Milano 1996.

-, Inclusion and Democracy, Oxford University Press, Oxford 2000.

Zagrebelsky G., Il diritto mite. Legge diritti giustizia, Einaudi, Torino 1992. Zamora D., Behrent M.C. (eds.), Foucault and Neoliberalism, Polity Press, Cambridge 2015.

Zanetti Gf., La nozione di giustizia in Aristotele, il Mulino, Bologna 1993.

Zolo D., La teoria comunista dell'estinzione dello Stato, De Donato, Bari 1974.

-, Il principato democratico. Per una teoria realistica della democrazia, Feltrinelli, Milano 1992.

- (a cura di), La cittadinanza. Appartenenza, identità, diritti, Laterza, Roma-Bari 1994. 


\section{MANUALI}

BiomedicA

Branchi R., Le impronte nel paziente totalmente edentulo

Branchi R., Riabilitazione protesica del paziente oncologico testa-collo

Martinelli E., Rieducazione del piede. Problematiche biomeccaniche e posturali statiche e dinamiche

Martinelli E., Sviluppo del dolore rachideo in gravidanza. Mutamenti della biomeccanica rachidea, problematiche posturali, prevenzione e attività fisica adattata pre e post parto

Martinelli E., Rieducazione posturale. Fondamenti per la progettazione della postura

Martinelli E., Prevenzione del mal di schiena di origine meccanica con attività motoria e comportamentale. Approfondimenti di patomeccanica e biomeccanica rachidea

Rossetti R., Manuale di batteriologia clinica. Dalla teoria alla pratica in laboratorio

Rucci L., Testo Atlante di embriologia clinica della Laringe. La chirurgia conservativa compartimentale della regione glottica

Rucci L., Clinical Embryology of $\mathrm{Hu}$ man Larynx for Conservative Compartmental Surgery. A Text and Atlas

ScIENZE

Bart J.C.J., Polymer Additive Analytics. Industrial Practice and Case Studies

Caramelli D., Antropologia molecolare. Manuale di base

Lo Nostro P., Peruzzi N., Spontaneamente. Esercizi Risolti di Chimica Fisica

Ricci R., Lezioni di Sistemi Dinamici, a cura di Lorenzo Barletti, Angiolo Farina, Lorenzo Fusi, Federico Talamucci

Scialpi A., Mengoni A. (a cura di), $L a$ PCR e le sue varianti. Quaderno di laboratorio

Simonetta M.A., Short history of Biology from the Origins to the 2oth Century

Spinicci R., Elementi di chimica (nuova edizione)
ScIEnze Sociali

Ciampi F., Fondamenti di economia e gestione delle imprese

Giovannini P. (a cura di), Teorie sociologiche alla prova

Maggino F., L'analisi dei dati nell'indagine statistica. Volume 1. La realizzazione dell'indagine e l'analisi preliminare dei dati

Maggino F., L'analisi dei dati nell'indagine statistica. Volume 2. L'esplorazione dei dati e la validazione dei risultati

Magliulo A., Elementi di economia del turismo

Marchettoni L., Breve storia della democrazia. Da Atene al populismo

Petretto A., Economics and Institutions and Law

Visentini L., Bertoldi, M., Conoscere le organizzazioni. Una guida alle prospettive analitiche $e$ alle pratiche gestionali

Scienze Tecnologiche

Borri C., Pastò S., Lezioni di ingegneria del vento

Borri C., Betti M., Marino E., Lectures on Solid Mechanics

Cidronali A., Paolo Colantonio P. e Lucci L., Antenne Integrate Attive

Gulli R., Struttura e costruzione / Structure and Construction

Maddio S., Introduzione ai Sistemi di Localizzazione Indoor

Policicchio F., Lineamenti di infrastrutture ferroviarie

UMANISTICA

Bertini F., Risorse, conflitti, continenti e nazioni. Dalla rivoluzione industriale alle guerre irachene, dal Risorgimento alla conferma della Costituzione repubblicana

Bombardieri L., Graziadio G., Jasink A.M., Preistoria e Protostoria egea e cipriota

Bombi A.S., Pinto G., Cannoni E., Pictorial Assessment of Interpersonal Relationships (PAIR). An analytic system for understanding children's drawings 
Baldini S., Marini D., Vorrei. Corso di lingua italiana di livello elementare 1. Libro di testo e libro degli esercizi

Borello E., Mannori S., Teoria e tecnica delle comunicazioni di massa

Brandi L., Salvadori B., Dal suono alla parola. Percezione e produzione del linguaggio tra neurolinguistica $e$ psicolinguistica

Coniglione F., Lenoci M., Mari G., Polizzi G. (a cura di), Manuale di base di Storia della filosofia

Marcialis N., Introduzione alla lingua paleoslava
Michelazzo F., Nuovi itinerari alla scoperta del greco antico. Le strutture fondamentali della lingua greca: fonetica, morfologia, sintassi, semantica, pragmatica

Peruzzi A., Il significato inesistente. Lezioni sulla semantica

Peruzzi A., Modelli della spiegazione scientifica

Sandrini M.G., Filosofia dei metodi induttivi e logica della ricerca

Trisciuzzi L., Zappaterra T., Bichi L., Tenersi per mano. Disabilità e formazione del sé nell'autobiografia 

